\author{
Universidade de São Paulo \\ Instituto de Física
}

\title{
Termodinâmica estocástica e eficiência para máquinas térmicas colisionais
}

\author{
Fernando Francisco Silva Filho
}

Orientador: Prof. Dr. Carlos Eduardo Fiore dos Santos

Dissertação de mestrado apresentada ao Instituto de Física da Universidade de São Paulo, como requisito parcial para a obtenção do título de Mestre em Ciências.

Banca Examinadora:

Prof. Dr. Ronald Dickman - UFMG

Prof. Dr. Beatriz Anteneodo de Porto - Puc-Rio

Prof. Dr. Carlos Eduardo Fiore dos Santos (Orientador) - IF-USP

São Paulo

2021 
FICHA CATALOGRÁFICA

Preparada pelo Serviço de Biblioteca e Informação do Instituto de Física da Universidade de São Paulo

Silva Filho, Fernando Francisco

Termodinâmica estocástica e eficiência para máquinas térmicas colisionais. São Paulo, 2021.

Dissertação (Mestrado) - Universidade de São Paulo. Instituto de Física. Depto. de Física Geral

Orientador: Prof. Dr. Carlos Eduardo Fiore dos Santos

Área de Concentração: Física

Unitermos: 1. Física; 2. Processos estocásticos; 3. Mecânica estatística.

USP/IF/SBI-058/2021 


\author{
University of São Paulo \\ Physics Institute
}

\title{
Stochastic thermodynamics and efficiency for thermal collisional engines
}

\author{
Fernando Francisco Silva Filho
}

Supervisor: Prof. Dr. Carlos Eduardo Fiore dos Santos

Dissertation submitted to the Physics Institute of the University of São Paulo in partial fulfillment of the requirements for the degree of Master of Science.

Examining Committee:

Prof. Dr. Ronald Dickman - UFMG

Prof. Dr. Beatriz Anteneodo de Porto - Puc-Rio

Prof. Dr. Carlos Eduardo Fiore dos Santos (Advisor) - IF-USP

São Paulo

2021 


\section{Dedicatória}

Dedico este trabalho a minha família, sem a qual nada seria possível. 


\section{Agradecimentos}

Em primeiro lugar, agradeço ao apoio e ao suporte prestados pela minha família. Também agradeço ao meu orientador, Carlos Fiore, pela paciência e pela amizade. Também devo agradeçer a todos os meus colegas e amigos do instituto e aos pesquisadores que colaboraram nas pesquisas aqui apresentadas. Muito Obrigado! O presente trabalho foi realizado com apoio da Coordenação de Aperfeiçoamento de Pessoal de Nível Superior - Brasil (CAPES) Código de Financiamento 001. 


\section{Resumo}

A performance de máquinas endoreversíveis em escalas mesoscópicas operando em potências finitas constitui um dos principais problemas da termodinâmica de não equilíbrio, tanto em sistemas clássicos quanto sistemas quânticos. Nesta dissertação de mestrado estudamos as propriedades termodinâmicas de modelos colisionais para máquinas térmicas em nanoescala compostas por "quantum dots"e por partículas Brownianas. No presente caso, a cada intervalo de tempo, o sistema está em contato com um diferente reservatório térmico e sujeito a forças externas distintas (no caso Browniano). As propriedades termodinâmicas são caracterizadas por meio da Termodinâmica Estocástica e as condições para a otimização da performance da máquina com relação a diferentes parâmetros (tempos assimétricos, forças e outras quantidades) são analisadas. Nossos resultados revelam que a presente abordagem constitui uma metodologia bastante adequada e útil para o estudo de máquinas térmicas operando a tempos finitos, em contraste com a máquina de Carnot que é bastante idealizada.

Palavras-chave: Termodinâmica estocástica; cadeias de markov; equações de FokkerPlanck; eficiência a máxima potência; nanoescala. 


\section{Abstract}

The performance of endoreversible thermal machines operating at finite power regime constitutes one of the main challenges of nonequilibrium thermodynamics, for classical and quantum systems. In this master's thesis we study the thermodynamic properties of collisional models of quantum dot and Brownian particle nanoscale thermal engines. In the present case, at each time interval, the system interact with a different thermal reservoir and under a different external force (in the Brownian case). The thermal properties are characterized using stochastic thermodynamics and the performance optimization of the engine in relation to different parameters (asymmetric times, forces and other quantities) is analyzed. Our results show that the present approach constitutes an adequate tool / methodology useful in the study of thermal engines working in finite time, in contrast to an idealized Carnot's machine.

Keywords: Stochastic thermodynamics; Markov chain; Fokker-Planck equation; efficiency at maximum power; nanoscale. 


\section{Conteúdo}

$\begin{array}{ll}\text { Dedicatória } & \text { i }\end{array}$

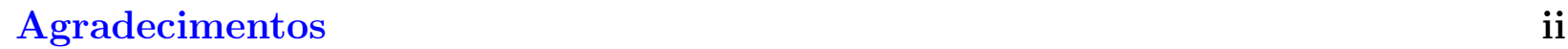

$\begin{array}{lll}\text { Resumo } & \text { iii }\end{array}$

Abstract $\quad$ iv

1 Introdução 1

2 Introdução à Termodinâmica Estocástica 5

2.1 Produção de Entropia . . . . . . . . . . . . . . . . . . . . 6

2.2 Coeficientes de Onsager f . . . . . . . . . . . . . . . . . . 10

2.3 Máquinas Térmicas e a Eficência de Curzon-Albohrn . . . . . . . . . . . . 11

2.4 Introdução aos Processos Estocásticos . . . . . . . . . . . . . . . . . 16

2.4 .1 Cadeias de Markov . . . . . . . . . . . . . . . . . . . 16

2.4.2 Estado Estacionário e o Teorema de Perron-Frobenius . . . . . . . . . 19 
2.4.3 Equação Mestra . . . . . . . . . . . . . . . . . 23

2.4.4 Produção de Entropia de Schnakenberg . . . . . . . . . . . . . . . . 27

2.5 Termodinâmica Estocástica para Processos Markovianos . . . . . . . . . . . 29

2.6 Equações de Fokker-Planck . . . . . . . . . . . . . . . . . . . . . 31

2.6 .1 Equações de Langevin . . . . . . . . . . . . . . . . . . . . 32

2.6.2 Termodinâmica Estocástica para Partículas Brownianas . . . . . . . . 34

3 Modelo Colisional para um Sistema de Quantum Dot 38

3.1 Modelo e Solução Exata . . . . . . . . . . . . . . . . . . . . . . 39

3.2 Descrição Termodinâmica . . . . . . . . . . . . . . . . . . 43

3.3 Protocolos de Maximização . . . . . . . . . . . . . . . . . . . . 45

3.4 Maximização Completa . . . . . . . . . . . . . . . . . . 53

4 Modelo Colisional para uma Partícula Browniana $\quad 58$

4.1 Modelo e Solução Exata . . . . . . . . . . . . . . . . . . . 59

4.2 Descrição Termodinâmica . . . . . . . . . . . . . . . . 61

4.2.1 Ausência de Forças Externas . . . . . . . . . . . . . . . . . 65

4.2.2 Forças Constantes . . . . . . . . . . . . . . . . 67

4.2.3 Forças Polinomiais .................. 73

4.2.4 Forças Senoidais . . . . . . . . . . . . . . 83

$\begin{array}{llr}5 & \text { Conclusão } & 89\end{array}$ 
A Artigo Publicado

Bibliografía 


\section{Capítulo 1}

\section{Introdução}

A termodinâmica estocástica constitui uma das principais áreas da mecânica estatística moderna e visa compreender fenômenos termodinâmicos presentes em escalas mesoscópicas, como partículas brownianas em banhos térmicos e sistemas descritos por equações mestras, propondo novas fronteiras e resultados que vão além do equilíbrio termodinâmico. Dentre eles, podemos citar a extensão de conceitos da termodinâmica de equilíbrio para sistemas fora do equilíbrio, calculando, por meio de modelos estocásticos, valores exatos para grandezas termodinâmicas como os fluxos de trabalho e de calor, considerações sobre máquinas térmicas, circuitos eletrônicos e como mitigar ou controlar a sua dissipação, eficiência e transições de fase. Em todos esses casos, o conceito de produção de entropia tem um papel central, sendo continuamente produzida em um estado estacionário de não equilíbrio (NESS). O NESS pode ser alcançado, em geral, de duas maneiras fundamentais: variações externas periódicas ou por forças termodinâmicas fixas.

A abordagem para o estudo de sistemas fora do equilíbrio se originou de diferentes abordagens, aplicações e contextos. Um dos mais notáveis envolve os trabalhos de Onsager [1] e Prigogine [2], que estabeleceram que próximo ao equilíbrio, fluxos termodinâmicos (calor, trabalho etc.) podem ser determinados por relações lineares em funções de correntes termodinâmicas. Outro notável resultado envolve a entropia total de um sistema em contato com um reservatório térmico, que varia continuamente em relação ao tempo e a sua taxa de 
variação pode ser escrita na forma

$$
\frac{d S}{d t}=\Pi-\Phi
$$

onde П é a produção de entropia, que deve ser sempre não negativa. A grandeza $\Phi$ refere-se ao fluxo de entropia fornecido (ou absorvido) pelo reservatório térmico. No estado estacionário de equilíbrio ambas as quantidades serão nulas, ao passo que ambas serão estritamente positivas no NESS, implicando que a produção de entropua distingue sistemas em equilíbrio dos sistemas fora do equilíbrio. Nos últimos anos, diversas implicações e aplicações sobre a produção de entropia têm sido consideradas. Destacamos as relações de flutuação, sua descrição para transições de fase e performance de máquinas térmicas e outros sistemas (condição de correntes elétricas e térmicas de dispositivos). Conforme mencionamos anteriormente, em muitos casos consideram-se forças termodinâmicas constantes e/ou aquelas sujeitas a variações periódicas. Nos últimos anos, modelos colisionais têm sido considerados em diferentes contextos, tanto no escopo da termodinâmica clássica, quanto quântica.

Modelos colisionais consistem em sistemas que interagem sequencialmente e repetidamente com diferentes ambientes térmicos (ao invés de interações contínuas com todos os reservatórios simultanementes). Estes modelos têm sido considerados os mais adequados para uma descrição mais realistas para diferentes sistemas que interagem com reservatórios térmicos, entre elas, podemos citar o caso de sistemas quânticos, onde o reservatório é convenientemente representado por uma sequência de partículas não correlaciondas [3]. Adicionalmente, o modelo colisional tenta descrever de maneira realista situações físicas onde partículas individuais interagem apenas com uma pequena fração do sistema, ou sistemas que envolvem interações com distintos drives externos [4]. Em particular, estudaremos máquinas colisionais cujo sistema interage sequencialmente e repetidamente com um reservatório térmico por vez, interagindo apenas com um tipo de drive em cada reservatório.

A eficiência de uma máquina térmica qualquer é limitada pela eficiência de Carnot, independente da sua configuração, sendo ela clássica ou quântica. Esse limite teórico foi estabelecido por Carnot em 1824 e consiste em um sistema que interage com duas istotermas e duas adiabáticas de maneira quasi-estática. Apesar desse limite ser um resultado central na termodinâmica, alcançá-lo é impraticável, já que para tal, o sistema seria obrigado a interagir de maneira reversível em cada etapa, implicando em uma interação lenta, levando a uma potência média nula (trabalho finito dividido por um intervalo de tempo muito grande). 
É desejável para máquinas térmicas reais que sua potência seja a maior possível (finita), mesmo que isso implique em uma diminuição da eficiência em relação a de Carnot devido a dissipações que ocorrem no ciclo. Surge daí o conceito de máquina endoreversível: cada etapa do ciclo termodinâmico é acelerada, gerando uma máquina com maior potência e disspação. Um dos resultados centrais para máquinas neste regime é a eficiência de Curzon-Ahlborn [5], um limite na eficiência de máquinas com potência máxima. Como discutido neste trabalho e em outros resultados já conhecidos na literatura, este limite não é universal como a eficiência de Carnot, apesar de ainda ser uma boa aproximação para máquinas nesse regime.

Nesta dissertação, estudaremos modelos colisionais para máquinas térmicas em nanoescalas compostas por "quantum dots"(QD) e por partículas Brownianas. Para o primeiro caso, consideramos um modelo para um quantum dot que interage sequencialmente com dois reservatórios térmicos e de partículas. Não há força externa aplicada a esse sistema e o trabalho útil extraído se deve à interação química entre o reservatório e o sistema, que doa ou recebe partículas. O segundo consiste em uma partícula browniana que interage sequencialmente com dois reservatórios térmicos. Neste último caso, há uma força externa da qual há extração de trabalho, enquanto que os reservatórios são responsáveis pelas interações térmicas.

Estudamos para ambos os casos protocolos para a maximização de eficiência e potência. Para o caso de QD, consideramos a assimetria no tempo de interação com os reservatórios (tempo de interação com um dos reservatórios diferente da metade do intervalo do ciclo completo) como uma forma de maximização e concluímos que essa forma de otimização é mais vantajosa que as maximizações em torno de outras grandezas físicas, como as taxas de ocupação de partículas no sistema. Adicionalmente, comparamos a eficiência da máquina em potência máxima com a eficiência de Curzon-Alborn, que pode ser ultrapassada.

Para o caso Browniano, o trabalho útil é extraído de uma força externa que colisionalmente se opoe a um fluxo termodinâmico na troca entre reservatórios. Assim, mesmo em reservatórios com diferenças pequenas de temperatura, é possível extrair potências com valores significativos. Em ambos os casos a distribuição de probabilidades é obtida de forma exata, via a equação mestra e a equação de Fokker-Planck, respectivamente, de forma que valores exatos das médias das grandezas termodinâmicas como o fluxo de calor e trabalho 
podem ser exatamente calculados. No segundo caso, podemos calcular e escrever a produção de entropia média em sua forma bilinear e encontrar os coeficientes de Onsager em termos das grandezas dos drives.

Ambos os estudos mostram perspectivas interessantes dentro da área de termodinâmica de não equilíbrio, incluindo uma discussão aprofundada da importância do tempo de interação do quantum dot com os reservatórios térmicos e a influência de diferentes expressões de forças externas na performance de máquinas brownianas. Além de nos prover uma validação e generalizações das limitações entre correntes e fluxos, como as desigualdades dos coeficientes de Onsager e as relações de incertezas termodinâmicas (thermodynamics uncertainty relations, TUR).

Esta dissertação está dividida da seguinte maneira: No Capítulo 2, introduzimos os principais conceitos de termodinâmica de não equilíbrio, partindo de argumentos macroscópicos e em seguida, os recuperamos a partir da descrição estocástica de modelos microscópicos, definindo as equações mestras e equações de Fokker-Planck. No Capítulo 3, introduzimos o modelo colisional para um sistema de quantum dot e estudamos a influência na assimetria no tempo na eficiência e na potência. No Capítulo 4, estudamos algumas formas para a força externa em máquinas brownianas e sua influência na performance dela. 


\section{Capítulo 2}

\section{Introdução à Termodinâmica Estocástica}

Os fenômenos termodinâmicos de um dado sistema podem ser descritos em termos do comportamento médio das partículas que o compõe [6,7]. A mecânica estatística de equilíbrio é muito bem sucedida ao introduzir uma abordagem baseada nos ensembles de Gibbs, satisfatória para essa descrição. Porém, nos últimos anos a termodinâmica de não equilíbrio e, em particular a Termodinâmica estocástica, têm feito avanços em descrever sistemas em escalas pequenas, em termos de suas trajetórias individuais e estendendo para o caso em que o sistema está sujeito a "forças"que o "tiram"do estado de equilíbrio termodinâmico. Na escala mesoscópica, o papel das flutuações nas quantidades termodinâmicas são importantes e precisam ser analisadas. Como exemplo, citamos partículas brownianas em banhos térmicos, máquinas moleculares, reações químicas fora do equilíbrio químico e sistemas descritos por equações mestras, com diversas "correntes microscópicas"sujeitas a diferentes drives. Neste capítulo, pretende-se inicialmente introduzir por meio de macroscópicos e fenomenológicos, conceitos básicos de Termodinâmica de não Equilíbrio. Também serão discutidos, de maneira geral, o conceito de máquinas térmicas em potência máxima e a eficiência de Curzon-Ahlborn. Finalmente, introduziremos a Termodinâmica Estocástica, reobtendo os resultados alcançados para o caso macroscópico, partindo de sistemas descritos por equações mestras e por equações de Fokker-Planck em escalas mesoscópicas. 


\subsection{Produção de Entropia}

O equilíbrio termodinâmico de um sistema isolado é determinado pelo princípio de máxima entropia [6,7]. Considere dois sistemas interagentes $A$ e $B$ compondo um sistema isolado $A+$ $B$ e sejam $\left\{X_{k}^{A}\right\}_{k=1, N}$ e $\left\{X_{k}^{B}\right\}_{k=1, N}$ o conjunto das $N$ variáveis extensivas, respectivamente, dos sistemas $A$ e $B$ (como, por exemplo, número de moles, volume e energia ). Dado um sistema isolado, a soma das grandezas extensivas de $A$ e $B$ devem ser constantes, logo:

$$
\begin{gathered}
X_{k}^{A}+X_{k}^{B}=\text { constante } ; \forall k=1, \cdots, N, \\
d X_{k}^{A}+d X_{k}^{B}=0 ; \quad \forall k=1, \cdots, N .
\end{gathered}
$$

Por outro lado, a entropia total não é conservada. Seu comportamento pode ser escrito em termos das grandezas extensivas e é dado por $S=S_{A}+S_{B}$. Para cada índice $k$, podemos encontrar a derivada parcial da entropia em relação a uma dada grandeza extensiva $X_{k}^{A}$, o que se identifica com a temperatura, pressão por temperatura ou potentical químico para $X_{k}=U, V, N$, respectivamente;

$$
\frac{\partial S}{\partial X_{k}^{A}}=\frac{\partial S_{A}}{\partial X_{k}^{A}}+\frac{\partial S_{B}}{\partial X_{k}^{A}}
$$

Uma vez que a soma das variáveis extensivas são conservadas e usando a regra da cadeia, podemos escrevrer que:

$$
\frac{\partial S_{B}}{\partial X_{k}^{A}}=\frac{d X_{k}^{B}}{d X_{k}^{A}} \frac{\partial S_{B}}{\partial x_{k}^{B}}=-\frac{\partial S_{B}}{\partial x_{k}^{B}},
$$

assim:

$$
\frac{\partial S}{\partial X_{k}^{A}}=\frac{\partial S_{A}}{\partial X_{k}^{A}}-\frac{\partial S_{B}}{\partial X_{k}^{B}}
$$

Pelo princípio de máxima entropia, o sistema estará em equilíbrio desde que a entropia total seja máxima, isto é $d S=0$. Expressando cada diferencial $d S_{A}$ e $d S_{B}$ em termos de $X_{k}$ 's, temos:

$$
\begin{aligned}
d S_{A} & =\sum_{j=1}^{N} \frac{\partial S_{A}}{\partial X_{j}^{A}} d X_{j}^{A}, \\
d S_{B} & =\sum_{j=1}^{N} \frac{\partial S_{B}}{\partial X_{j}^{B}} d X_{j}^{B},
\end{aligned}
$$

a diferencial da entropia $S$ do sistema é reescrita como:

$$
d S=\sum_{j=1}^{N}\left(\frac{\partial S_{A}}{\partial X_{j}^{A}} d X_{j}^{A}+\frac{\partial S_{B}}{\partial X_{j}^{B}} d X_{j}^{B}\right) .
$$


Pela relação (2.1.2), podemos escrever:

$$
d S=\sum_{j=1}^{N}\left(\frac{\partial S_{A}}{\partial X_{j}^{A}}-\frac{\partial S_{B}}{\partial X_{j}^{B}}\right) d X_{j}^{A}=\sum_{j=1}^{N}\left(\frac{\partial S_{B}}{\partial X_{j}^{B}}-\frac{\partial S_{A}}{\partial X_{j}^{A}}\right) d X_{j}^{B}
$$

Conforme mencionado anteriormente, as grandezas extensivas estão associadas a uma dada grandeza intensiva, aqui expressa por $f_{k}$. No equilíbrio, é estabelecido que as grandezas intensivas para cada índice $k$ sejam iguais. Logo:

$$
f_{k}^{A}=f_{k}^{B}, \text { para todo } k=1, \cdots, N
$$

Isto é consistente com a igualdade entre as temperaturas, pressões e potencians químicos se $X_{k}=U, V, N$, respectivamente.

Vamos considerar agora um processo dinâmico em torno do equilíbrio de tal modo que a evolução da entropia e das grandezas extensivas sejam descritas como funções diferenciáveis em relação ao tempo. Dada a expressão da diferencial da entropia (2.1.5), a derivada no tempo é dada por:

$$
\frac{d S}{d t}=\sum_{j=1}^{N}\left(\frac{\partial S_{A}}{\partial X_{j}^{A}} \dot{X}_{j}^{A}+\frac{\partial S_{B}}{\partial X_{j}^{B}} \dot{X}_{j}^{B}\right)
$$

onde $\dot{X}_{k}^{A}$ e $\dot{X}_{k}^{B}$ são as derivadas temporais das grandezas extensivas. Como o sistema $A+B$ é isolado e portanto, esse processo dinâmico ocorre por meio de interações termodinâmicas entre $A$ e $B$. Desta forma, considerando a expressão (2.1.2), temos que:

$$
\dot{X}_{k}^{A}=-\dot{X}_{k}^{B}
$$

de onde reescrevemos:

$$
\frac{d S}{d t}=\sum_{j=1}^{N}\left(\frac{\partial S_{A}}{\partial X_{j}^{A}}-\frac{\partial S_{B}}{\partial X_{j}^{B}}\right) \dot{X}_{j}^{A}
$$

Podemos definir as correntes termodinâmicas como as taxas de variação das grandezas extensivas ao longo do processo. Façamos a corrente/fluxo $J_{k}=\dot{X}_{k}^{A}$. Também podemos definir a afinidade termodinâmica ou a força termodinâmica por $\mathcal{F}_{k}:=\frac{\partial S_{A}}{\partial X_{k}^{A}}-\frac{\partial S_{B}}{\partial X_{k}^{B}}$ que expressa a diferença entre as grandezas intensivas. Podemos escrever a taxa de variação da entropia como:

$$
\frac{d S}{d t}=\sum_{j=1}^{N} \mathcal{F}_{j} J_{j}
$$


A relação acima mostra que a taxa de variação de entropia total (ou produção de entropia) pode ser expressa como a soma dos produtos da "força"(ou afinidade) com a "corrente". Considerando que o sistema esteja isolado, sabemos que o mesmo evolui para o equilíbrio e, nesta condição, as grandezas extensivas não variam no tempo e portanto $\dot{X}_{k}^{A}=-\dot{X}_{k}^{B}=0$. Assim, as grandezas intensivas de $A$ e $B$ serão iguais e podemos interpretar fisicamente a afinidade $\mathcal{F}_{k}$ como o fator responsável pelas mudanças das grandezas extensivas. Ou seja, a diferença entre $f_{k}^{A}$ e $f_{k}^{B}$ é responsável pela variação de $X_{k}^{A}$ e $X_{k}^{B}$.

A título de ilustração, podemos considerar um sistema termo-mecânico isolado $A+B$ que evolui para o equilíbrio. Podemos escrever as grandezas extensivas (energia interna e volume) desse sistema como:

$$
\begin{aligned}
& U=U_{A}(t)+U_{B}(t), \\
& V=V_{A}(t)+V_{B}(t),
\end{aligned}
$$

onde o volume $V$ e a energia interna $U$ são constantes. Nesse caso, a entropia evoluirá no tempo até o seu valor máximo. Considerando a expressão (2.1.8), podemos escrever a taxa de variação de entropia como:

$$
\frac{d S}{d t}=\mathcal{F}_{U} J_{U}+\mathcal{F}_{V} J_{V}
$$

onde:

$$
\begin{array}{cc}
\mathcal{F}_{U}=f_{U}^{A}-f_{U}^{B} \quad \mathcal{F}_{V}=f_{V}^{A}-f_{V}^{B}, \\
J_{U}=\dot{U}_{A} \quad J_{V}=\dot{V}_{A},
\end{array}
$$

são as afinidades e as correntes do sistema. Como sabemos, as grandezas intensivas relacionadas a cada grandeza extensiva são dadas por:

$$
\begin{gathered}
f_{U}^{A, B}=\frac{\partial S_{A, B}}{\partial U_{A, B}}=\frac{1}{T_{A, B}}, \\
f_{V}^{A, B}=\frac{\partial S_{A, B}}{\partial V_{A, B}}=\frac{P_{A, B}}{T_{A, B}},
\end{gathered}
$$

onde $T$ e $P$ são as temperaturas e as pressões dos respectivos componentes $A$ e $B$. Assim, podemos escrever:

$$
\frac{d S}{d t}=\left(\frac{1}{T_{A}}-\frac{1}{T_{B}}\right) \dot{U}_{A}+\left(\frac{P_{A}}{T_{A}}-\frac{P_{B}}{T_{B}}\right) \dot{V}_{A} .
$$


Os equilíbrios mecânicos e térmicos são alcançados, respectivamente, quando:

$$
\begin{gathered}
\frac{P_{A}}{T_{A}}=\frac{P_{B}}{T_{B}}, \\
\frac{1}{T_{A}}=\frac{1}{T_{B}} .
\end{gathered}
$$

A condição para o equilíbrio total do sistema ocorre quando as afinidades são nulas para cada índice $k$. Ou seja, para cada $k=1, \ldots, N, \mathcal{F}_{k}=0$. Por outro lado, se $\mathcal{F}_{k} \neq 0$, o sistema evoluirá para um estado estacionário de não equilíbrio em que a variação de entropia $\frac{d S}{d t}$ é estritamente positiva. Como o sistema $A+B$ é isolado, não há um fluxo de entropia do sistema para o meio externo e podemos associar o aumento de entropia com uma produção de entropia interna. Tal produção não ocorre exclusivamente por $A$ ou $B$, mas é uma propreidade de $A+B$ devido à troca de calor irreversível entre eles.

Um caso particular muito importante é aquele que envolve a interação de um sistema com um reservatório. Seja a interação $A+B$ uma composição isolada como definida acima, podemos considerar $B$ um reservatório térmico considerando $B$ muito maior que $A$. Dessa forma, as variações das grandezas intensivas de $B$ devido a interação são muito menores em comparação com as variações de $A$, de modo que podemos considerar $f_{k}^{B}$ para cada $k$ constante no tempo. Nosso objetivo é estudar a evolução do sistema $A$ considerando a sua interação com o reservatório $B$. A taxa de variação da entropia total do sistema é dado por:

$$
\frac{d S}{d t}=\frac{d S_{A}}{d t}+\frac{d S_{B}}{d t}
$$

assim, reescrevemos a derivada de $S_{A}$ como:

$$
\frac{d S_{A}}{d t}=\frac{d S}{d t}-\frac{d S_{B}}{d t}
$$

Pelo princípio da máxima entropia, a entropia total do sistema evolui para o máximo ao longo do tempo, o que implica que sua derivada temporal é sempre positiva. Ou seja:

$$
\frac{d S}{d t} \geq 0
$$

Por outro lado, o sinal da contribuição de $B$ pode variar entre positivo ou negativo, dependendo da natureza da interação entre $A$ e $B$. Podemos reescrever a expressão (2.1.15) como:

$$
\dot{S}_{A}=\Pi-\Phi,
$$


onde:

$$
\Pi=\frac{d S}{d t} \geq 0
$$

é, conforme mencionamos anteiormente, chamada de produção de entropia, um termo sempre maior ou igual a zero. Em particular, a produção de entropia é nula apenas no equilíbrio. Por outro lado, o termo:

$$
\Phi=\frac{d S_{B}}{d t}
$$

é determinado por fluxo de entropia e expressa o fluxo de entropia do sistema para o reservatório e portanto, pode ser tanto positiva quanto negativa. Essa mesma relação será encontrada em termos de argumentos estocásticos nos próximos capítulos, tanto para o Markoviano, tanto para o caso descrito por equações de Langevin.

\subsection{Coeficientes de Onsager}

Considere a interação termodinâmica entre $A$ e $B$ como considerado acima. Para cada afinidade $\mathcal{F}_{k}$ não nula, existe uma corrente $\dot{X}_{k}$. Conforme proposto por Onsager [1], quando há diferentes interações relacionadas a diferentes afinidades, uma em geral influencia na outra. Isto é, podemos escrever cada corrente $\dot{X}_{k}$ em função de todas as afinidades que a "interferem" $\left\{\mathcal{F}_{j}\right\}$. Em particular, no equilíbrio, as afinidades são nulas e portanto a corrente $\dot{X}_{k}\left(\left\{\mathcal{F}_{j}\right\}\right)=0$. Para regimes próximos ao equilíbrio, $\mathcal{F}_{k}=f_{k}^{A}-f_{k}^{B} \approx 0$ para todo $k$, de forma que podemos escrever $\dot{X}_{k}$ em termos de uma expansão de Taylor até a primeira ordem em torno do equilíbrio $\left(\mathcal{F}_{k}=0\right.$ para todo $\left.k\right)$ :

$$
J_{k}=\sum_{n} L_{k n} \mathcal{F}_{n}
$$

onde $L_{n k}$ são chamadas de coeficientes de Onsager [1]. Dada a expressão (2.1.9), podemos escrever a produção de entropia dada por:

$$
\Pi=\sum_{m} \mathcal{F}_{m} J_{m}=\sum_{m} \sum_{n} L_{m n} \mathcal{F}_{n} \mathcal{F}_{m} \geq 0
$$

A expressão (2.2.2) sugere que a produção de entropia pode ser escrita em uma forma quadrática (bilinear). Considerando a Segunda Lei da Termodinâmica, a produção de entropia é 
uma forma quadrática não negativa. A título de exemplo, vamos considerar duas afinidades $\mathcal{F}_{1}$ e $\mathcal{F}_{2}$. Assim, as correntes são dadas por:

$$
\begin{aligned}
& J_{1}=L_{11} \mathcal{F}_{1}+L_{12} \mathcal{F}_{2}, \\
& J_{2}=L_{21} \mathcal{F}_{1}+L_{22} \mathcal{F}_{2},
\end{aligned}
$$

e a produção de entropia é dada por

$$
\Pi=L_{11} \mathcal{F}_{1}^{2}+L_{22} \mathcal{F}_{2}^{2}+\left(L_{12}+L_{21}\right) \mathcal{F}_{1} \mathcal{F}_{2} .
$$

Como discutido anteriormente, $\Pi \geq 0$. Isso implica nas seguintes relações para $L_{i j}$ :

$$
\left\{\begin{array}{l}
L_{11}>0, L_{22}>0, \\
4 L_{11} L_{22}-\left(L_{12}+L_{21}\right)^{2} \geq 0 .
\end{array}\right.
$$

No caso de forças constantes, Onsager mostrou a igualdade entre os coeficientes (relação de reciprocidade de Onsager) [1]:

$$
L_{12}=L_{21}
$$

Por outro lado, no caso de forças periódicas ou variantes no tempo, em geral a relação acima não se aplica e é substituída por uma relação mais fraca, $L_{21}=\tilde{L}_{12}$, onde o índice $\sim$ corresponde à simetria em relação à inversão temporal. A definição de reversibilidade microscópica e sua interpretação em termos estocásticos serão discutidas ao longo das próximas seções.

\subsection{Máquinas Térmicas e a Eficência de Curzon-Albohrn}

A eficiência de Carnot é sem dúvida um dos principais resultados da Termodinâmica. Ela estabelece que qualquer máquina térmica sempre terá como o limite superior da eficência o rendimento de uma máquina de Carnot $\eta_{\mathrm{C}}$ operando nas mesmas temperaturas:

$$
\eta \leq \eta_{\mathrm{C}}=1-\frac{T_{\mathrm{C}}}{T_{\mathrm{H}}}
$$

onde $T_{\mathrm{C}}$ e $T_{\mathrm{H}}$ são, respectivamente, as temperaturas em escala absoluta das fontes fria e quente que regem a máquina. Tal limite foi introduzido pelo engenheiro francês Sadi Carnot em 1824 [12] e consiste em uma máquina reversível composta por dois processos quasiestáticos adiabáticos e isotermos formando um ciclo. O limite estabelecido pela desigualdade 
(2.3.1) independe da natureza, formato ou do tipo de ciclo realizado pela máquina, sendo ela clássica ou quântica $[7,13]$. Porém, alcançá-la é impraticável, já que para operar em um ciclo reversível, seu processo deve ser quasi-estático, implicando uma potência nula (trabalho total finito realizado pela máquina dividido por um tempo muito longo).

Um modelo mais realista para uma máquina térmica busca um limite de ciclos rápidos, tornando a máquina "irreversível"e aumentando dessa forma a dissipação, diminuindo assim sua eficiência. Nessas condições, é possível maximizar a potência de saída (finitamente) e encontrar seu rendimento neste regime. Operando nessas condições, o regime é chamado de endoreversível. Este procedimento foi realizado para ciclos em reatores nuclear por Chambadal [14] em 1957 e por Novikov [15] em 1958, feito de maneira mais geral para ciclos termodinâmicos pelos físicos Curzon e Ahlborn em 1975 [5]. Tais trabalhos encontraram um novo limite para a eficência de uma máquina no regime de máxima potência, a eficiência de Curzon-Ahlborn (ou Chambadal-Novikov), que será resumidamente obtida a seguir:

$$
\eta_{\mathrm{CA}}=1-\sqrt{\frac{T_{\mathrm{C}}}{T_{\mathrm{H}}}} .
$$

A ideia inicial para se aumentar a potência útil de um ciclo de Carnot é acelerar o intervalo de tempo de cada etapa da máquina. Porém, se o ciclo for muito rápido, as interações térmicas entre o sistema e as isotermas não têm tempo suficiente para mudar a temperatura da substância que compoe a máquina, resultando em uma potência pequena e uma eficiência quase nula. Assim, entre os extremos de ciclos lentos e rápidos, existe um intervalo de tempo ótimo que maximiza a potência do ciclo de maneira finita [5].

Sejam $T_{1}$ e $T_{2}$ as temperaturas dos reservatórios, com $T_{1}>T_{2}$ e $T_{1 S}$ e $T_{2 S}$ as temperaturas da substância que compoe máquina ao interagir respectivamente com os reservatórios de temperaturas $T_{1}$ e $T_{2}$. Curzon e Ahlborn consideram que o fluxo de calor trocado entre cada isoterma seja proporcional às diferenças de temperatura entre a substância e o reservatório, assim:

$$
\begin{aligned}
& F_{1}=\alpha\left(T_{1}-T_{1 S}\right), \\
& F_{2}=\beta\left(T_{2 S}-T_{2}\right),
\end{aligned}
$$

onde $\alpha$ e $\beta$ são constantes positivas com unidades de energia por tempo e temperatura. Definindo o intervalo de tempo da interação entre o sistema e os reservatórios como $\Delta t_{1} \mathrm{e}$ 
$\Delta t_{2}$, conseguimos escrever o calor total trocado entre o sistema e os reservatórios como:

$$
\begin{aligned}
& \Delta Q_{1}=F_{1} \Delta t_{1}=\alpha \Delta t_{1}\left(T_{1}-T_{1 s}\right), \\
& \Delta Q_{2}=F_{2} \Delta t_{2}=\beta \Delta t_{2}\left(T_{2 S}-T_{2}\right) .
\end{aligned}
$$

Além disso, como as etapas adiabáticas são também isentrópicas, temos que:

$$
\frac{\Delta Q_{1}}{T_{1}}=\frac{\Delta Q_{2}}{T_{2}} .
$$

Podemos considerar que o tempo total do ciclo $\tau$ é proporcional à duração das interações do sistema com as isotermas do sistema. Isto é:

$$
\tau=\gamma\left(\Delta t_{1}+\Delta t_{2}\right)
$$

para o caso de máquinas colisionais, as interações adiabáticas são consideradas instantâneas, de modo que $\gamma \approx 1$. Seja $\Delta W$ o trabalho útil extraído da máquina (área do ciclo de Carnot no diagrama Clapeyron). Pela primeira lei da termodinâmica, podemos escrever $\Delta W=\Delta Q_{1}-\Delta Q_{2}$. Assim, com base nas expressões (2.3.5 - 2.3.7) a potência extraída em um ciclo é dada por:

$$
P=\frac{\Delta Q_{1}-\Delta Q_{2}}{\tau}=\frac{\Delta Q_{1}-\Delta Q_{2}}{\gamma\left(\Delta t_{1}+\Delta t_{2}\right)}
$$

Usando a equação (2.3.7), podemos eliminar a variável de tempo na expressão da potência e reescrevê-la em termos das varáveis $x=T_{1}-T_{1 S}$ e $y=T_{2 S}-T_{2}$ :

$$
P(x, y)=\frac{\alpha \beta x y\left(T_{1}-T_{2}-x-y\right)}{\gamma\left(\beta T_{1} y+\alpha T_{2} x+x y(\alpha-\beta)\right)} .
$$

Fisicamente, esperamos que $P(x, y) \geq 0$. Além disso, como discutimos antes, a potência será nula para valores de $x$ e $y$ próximos de zero e para valores muito grandes dessas variáveis. Assim, esperamos que a função $P(x, y)$ seja côncava, tendo como seu único ponto crítico $\left(x_{0}, y_{0}\right)$, o ponto de máximo. Assim, para encontrá-lo, basta encontrar $x_{0}$ e $y_{0}$ com as derivadas parciais:

$$
\left.\frac{\partial P}{\partial x}\right|_{(x, y)=\left(x_{0}, y_{0}\right)}=\left.\frac{\partial P}{\partial y}\right|_{(x, y)=\left(x_{0}, y_{0}\right)}=0 .
$$

Após certa álgebra, a equação acima pode ser reduzida no seguinte sistema:

$$
\begin{aligned}
& \beta T_{1} y_{0}\left(T_{1}-T_{2}-x_{0}-y_{0}\right)=x_{0}\left(\beta T_{1} y_{0}+\alpha T_{2} x_{0}+x_{0} y_{0}(\alpha-\beta)\right), \\
& \alpha T_{2} x_{0}\left(T_{1}-T_{2}-x_{0}-y_{0}\right)=y_{0}\left(\beta T_{1} y_{0}+\alpha T_{2} x_{0}+x_{0} y_{0}(\alpha-\beta)\right) .
\end{aligned}
$$


Para $y_{0}$, encontramos:

$$
y_{0}=\sqrt{\frac{\alpha T_{2}}{\beta T_{1}}} x_{0},
$$

restando uma equação quadrática para $x_{0}$, tendo duas soluções distintas. A solução correta para o nosso caso é aquela em que $x_{0}>0$, já que o sistema opera como máquina se $x=$ $T_{1}-T_{1 s}>0$. Encontramos portanto:

$$
x_{0}=\frac{T_{1}\left(1-\sqrt{T_{1} / T_{2}}\right)}{1+\sqrt{\alpha / \beta}} .
$$

Assim, a eficiência da máquina pode ser escrita como:

$$
\eta=1-\frac{\Delta Q_{1}}{\Delta Q_{2}}=1-\frac{T_{2 S}}{T_{1 S}}
$$

substituindo os valores encontrados em $(2.3 .14,2.3 .15)$, temos:

$$
\eta=1-\frac{T_{2}+y_{0}}{T_{1}-x_{0}}=1-\sqrt{\frac{T_{2}}{T_{1}}},
$$

que reproduz a expressão (2.3.2). Apesar desse limite não ter o mesmo caráter de universalidade da eficiência de Carnot [16,17], a expressão (2.3.2) é uma boa aproximação para a eficiência em regimes mais realistas. Evidentemente, $\eta_{\mathrm{CA}}<\eta_{\mathrm{C}}$. Além disso, podemos reescrever a expressão (2.3.2) como:

$$
\eta_{C A}=1-\sqrt{1-\eta_{C}}
$$

que pode ser expandida em séries de Taylor, para valores de $\eta_{C}$ em torno do zero:

$$
\eta_{C A}=\frac{1}{2} \eta_{C}+\frac{1}{4} \eta_{C}^{2}+\frac{3}{8} \eta_{C}^{3}+\cdots
$$

Considerando um caso onde $\eta_{C} \approx 0\left(T_{C} \approx T_{H}\right)$, a eficiência de CA pode ser aproximada por:

$$
\eta_{C A} \approx \frac{1}{2} \eta_{C}
$$

Por outro lado, ao considerar um valor de $\eta_{C}$ próximo de $1\left(T_{C}<<T_{H}\right)$ :

$$
\eta_{C A} \approx \eta_{C}
$$

Neste trabalho, estudaremos as ditas máquinas colisionais, que consistem em sistemas termodinâmicos que interagem com dois ou mais reservatórios sequencialmente, sendo a mudança de um para outro instantânea e em geral associada à etapa adiabática do ciclo. Ao final do 
mesmo, o sistema volta ao reservatório inicial e repete a sequência. A mudança de reservatório deve ocorrer por meio de uma perturbação externa que realiza a troca. O esquema apresentado na figura (2.1) a seguir e ilustra esse processo para um caso onde há dois reservatórios.

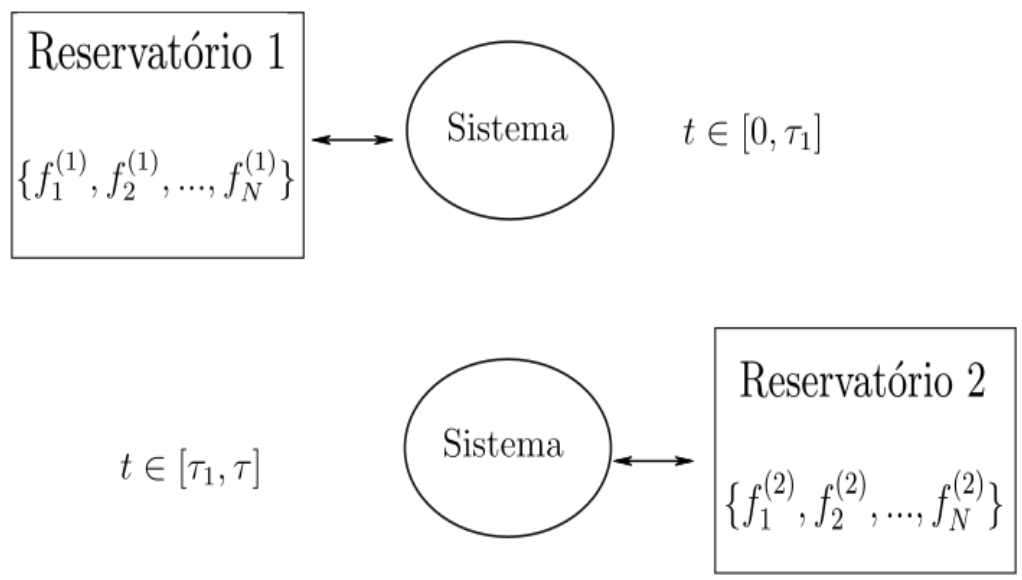

Figura 2.1: Modelo esquemático de uma máquina colisional. Inicialmente, o sistema interage com um reservatório com as grandezas intensivas dadas por $f_{k}^{(1)}$ durante o intervalo de tempo $\tau_{1}$. Após este intervalo, o sistema interage com o segundo reservatório, durante um intervalo de tempo dado por $\tau_{2}=\tau-\tau_{1}$. Após um ciclo com duração $\tau$, o sistema volta a interagir com o primeiro reservatório.

Máquinas térmicas em escala macroscópica envolvem partes móveis, como pistões empurrados por fluidos. Este esquema é difícil de se reproduzir em escalas menores e não descreve ciclos termodinâmicos em nanoescala com exatidão. Nessas escalas, ciclos termodinâmicos são experimentalmente constrúidos com fluxos de partículas (como eletrons) entre reservatórios e forças externas sendo exercidas em partículas individuais, que extraem trabalho útil [23]. Parte central deste trabalho envolve modelar esses processos, estudando máquinas térmicas em escala mesoscópica. A seguir, introduziremos os conceitos de termodinâmica estocástica e abordaremos suas aplicações em escalas microscópicas. 


\subsection{Introdução aos Processos Estocásticos}

Dado um índice $\mathcal{T}=\{0,1,2, \ldots\}$ (em geral associado ao tempo), um processo estocástico indexado por $\mathcal{T}$ é uma coleção dada por $\left\{x_{t}, t \in \mathcal{T}\right\}$, onde $x_{t}$ é uma variável aleatória para cada $t \in \mathcal{T}$, com uma distribuição de probabilidade $P_{t}\left(x_{t}\right)$ bem definida. Para cada valor $l \in \mathcal{T}$, o conjunto $\left\{n_{0}, n_{1}, n_{2}, \ldots, n_{l}\right\}$ tem distribuição de probabilidade definida por $P_{l}\left(n_{0}, n_{1}, \ldots, n_{l}\right)$, onde $n_{j}$ são valores das variáveis $x_{j}$ assumidos no tempo $j$. Ou seja, para dado $l$, a probabilidade das variáveis aleatórios assumirem os valores $n_{j}$ para $j \in\{0,1, \ldots, l\}$ é dada por $P_{l}\left(n_{0}, n_{1}, \ldots, n_{l}\right)$. Consideraremos a marginalização desta distribuição, isto é, a probabilidade de cada estado $n_{j}$ é dada pela somatória:

$$
P\left(n_{j}\right)=\sum_{n_{0}} \cdots \sum_{\left(n_{j}\right)} \cdots \sum_{n_{l}} P_{l}\left(n_{0}, \cdots, n_{l}\right) \text { e } \sum_{n_{j}} P\left(n_{j}\right)=1
$$

onde a primeira somatória ocorre para todos os valores de $i$, exceto $i=j$. Dado um conjunto de valores para $x_{j}$ no intervalo de tempo $t=l \in\left\{n_{0}, n_{1}, \ldots, n_{l}\right\}$, a probabilidade condicional do valor $n_{l+1}$ ser assumido após os valores de $n$ dados é escrita como [9]:

$$
P_{l+1}\left(n_{l+1} \mid n_{0}, n_{1}, \ldots, n_{l}\right)=\frac{P_{l+1}\left(n_{0}, n_{1}, \ldots, n_{l+1}\right)}{P_{l}\left(n_{0}, n_{1}, \ldots, n_{l}\right)} .
$$

\subsubsection{Cadeias de Markov}

Considere um processo estocástico nas mesmas condições definidas acima. Chamamos este processo de markoviano (ou de cadeia de Markov) se, para todo $l \in \mathcal{T}$ e para uma dada sequência de eventos aleatórios $\left\{n_{0}, n_{1}, . ., n_{l}\right\}$, a igualdade a seguir for válida:

$$
P_{l+1}\left(n_{l+1} \mid n_{0}, n_{1}, \ldots, n_{l}\right)=P_{l+1}\left(n_{l+1} \mid n_{l}\right) .
$$

Portanto, em um processo markoviano, a probabilidade da mudança de estado $n_{l} \rightarrow n_{l+1}$ independe dos valores assumidos por $x_{j}$ para $j<l$, dependendo apenas do estado $n_{l}$ (processo sem "memória"). Considerando a expressão (2.4.2), escrevemos:

$$
P_{l+1}\left(n_{l+1} \mid n_{0}, \ldots, n_{l}\right) P_{l}\left(n_{0}, \ldots, n_{l}\right)=P_{l+1}\left(n_{0}, \ldots, n_{l+1}\right) .
$$

Partindo da definição de cadeia de Markov, podemos reescrever a expressão:

$$
P_{l+1}\left(n_{l+1} \mid n_{l}\right) P_{l}\left(n_{0}, \ldots, n_{l}\right)=P_{l+1}\left(n_{0}, \ldots, n_{l+1}\right) .
$$


A equação acima é válida para todo $l \in\{0,1,2, \ldots\}$. Portanto, podemos escrever de maneira recursiva:

$$
\begin{aligned}
P_{l}\left(n_{0}, \ldots, n_{l}\right) & =P_{l}\left(n_{l} \mid n_{l-1}\right) P_{l-1}\left(n_{0}, \ldots, n_{l-1}\right), \\
P_{l-1}\left(n_{0}, \ldots, n_{l-1}\right) & =P_{l-1}\left(n_{l-1} \mid n_{l-2}\right) P_{l-2}\left(n_{0}, \ldots, n_{l-2}\right), \\
& \cdots \\
P_{1}\left(n_{0}, n_{1}\right) & =P_{1}\left(n_{1} \mid n_{0}\right) P_{0}\left(n_{0}\right) .
\end{aligned}
$$

Isto é, para cada $l+1$, a probabilidade $P_{l+1}\left(n_{0}, \ldots, n_{l+1}\right)$ fica completamente definida pelas probabilidades de transição e pela probabilidade inicial, como expresso a seguir:

$$
P_{l}\left(n_{0}, \ldots, n_{l}\right)=P_{l}\left(n_{l} \mid n_{l-1}\right) P_{l-1}\left(n_{l-1} \mid n_{l-2}\right) \ldots P_{1}\left(n_{1} \mid n_{0}\right) P_{0}\left(n_{0}\right) .
$$

Além disso, considerando a marginalização da probabilidade (2.4.1), podemos escrever:

$$
P_{l}\left(n_{l}\right)=\sum_{n_{0}} \cdots \sum_{n_{l-1}} P_{l}\left(n_{l} \mid n_{l-1}\right) P_{l-1}\left(n_{l-1} \mid n_{l-2}\right) \ldots P_{1}\left(n_{1} \mid n_{0}\right) P_{0}\left(n_{0}\right)
$$

O somatório acima resulta em:

$$
P_{l}\left(n_{l}\right)=\sum_{n_{l-1}} P_{l-1}\left(n_{l} \mid n_{l-1}\right) P_{l-1}\left(n_{l-1}\right)
$$

A probabilidade condicional $P_{n-1}\left(n_{l} \mid n_{l-1}\right)$ é chamada de probabilidade de transição. Considerálo-emos constantes no tempo para todo instante $l-1$. Substituindo os estados $n_{l} \rightarrow m$ e $n_{l-1} \rightarrow n$, a probabilidade $P(m \mid n)=T_{m n}$ expressa a probabilidade de transição entre $n \rightarrow$ $m$ e a expressão (2.4.9) é reescrita como:

$$
P_{l}(m)=\sum_{n} T_{m n} P_{l-1}(n)
$$

que por sua vez pode ser reescrito na forma matricial:

$$
P_{l}=T P_{l-1}
$$

onde $P_{l}$ e $P_{l-1}$ são as matrizes coluna com as probabilidades, portanto suas entradas são sempre não negativas e a soma de seus elementos é igual a um e $T=\left[T_{i j}\right]$ é uma matriz quadrada com as seguintes propriedades:

$$
\left\{\begin{array}{l}
T_{i j} \geq 0, \forall i, j \\
\sum_{i} T_{i j}=1, \forall j
\end{array}\right.
$$


A segunda propriedade é devida à normalização das probabilidades condicionais. A matriz $T$ é chamada de matriz estocástica ou matriz de transição. Sendo a expressão (2.4.11) válida para todo $l$, podemos escrever:

$$
\begin{aligned}
P_{1} & =T P_{0}, \\
P_{2} & =T P_{1}, \\
& \cdots \\
P_{l} & =T P_{l-1},
\end{aligned}
$$

e de maneira recursiva, temos:

$$
P_{l}=T P_{l-1}=T^{2} P_{l-2}=\ldots=T^{l} P_{0}
$$

logo:

$$
P_{l}=T^{l} P_{0}
$$

Para descrever $P_{l}$ em qualquer instante $l$, basta que saibamos a probabilidade inicial $P_{0}$ e a matriz estocástica $T$.

Uma pergunta que naturalmente surge sobre as probabilidades de transição em $T$ é se todo estado $n$ pode ser acessado partindo de um estado $m$ qualquer após um número finito de passos $l$. Aqui, denotaremos $T_{i j}^{l}$ o elemento da matriz estocástica $T^{l}$. Um estado $n$ só pode ser atingido a partir de um estado $m$ no instante $l$ se e somente se $T_{n m}^{l} \neq 0$. Dizemos que a matriz de transição $T$ é irredutível se para um dado par $m, n, \exists l>0$ tal que $T_{n m}^{l}>0$. Quando a matriz não obedece a essa propriedade, ela é chamada de redutível. Considere um caso descrito por uma matriz $T$ irredutível que tem todos os seus elementos estritamente positivo para todos os pares $(m, n)$ Este caso particular de uma matriz irredutível é chamada de matriz regular: Seja $T$ uma matriz estocástica (que obedece as propriedades (2.4.12)) irredutível. $T$ é uma matriz estocástica regular se, e somente se, $\exists \mathrm{l}>0$ finito tal que $T_{n m}^{l}>0$ para todo o par $(n, m)$. Em termos gerais, sendo $T$ uma matriz regular, todo estado $m$ é acessível partindo de um estado $n$ para um dado número de passos $l$. 


\subsubsection{Estado Estacionário e o Teorema de Perron-Frobenius}

Suponha uma matriz estocástica regular $T$ e um estado inicial $P_{0}$. Estamos interessados em uma solução estacionária da equação (2.4.15) alcançada para tempos longos. Isto é:

$$
\begin{gathered}
\lim _{t \rightarrow \infty} P_{l}=P, \\
T P=P .
\end{gathered}
$$

A solução estacionária $P$, pela equação (2.4.17), é um autovetor de $T$, com autovalor igual a 1. Naturalmente, surge daí uma indagação sobre a existência e unicidade de um estado estacionário para uma dada matriz regular T. Inicialmente, para garantir a unicidade, o autovalor 1 deve ser não degenerado. Além disso, dado uma matriz estocástica $T$ com autovalor igual a 1, seu respectivo autovetor deve ter apenas entradas positivas e deve ser normalizado, representando, portanto, um vetor de probabilidades. Esse resultado é garantido pelo o Teorema de Perron-Frobenius [9]. Para cada matriz regular, existe um autovalor $\lambda_{0}=1$ não degenerado, com o único autovetor sendo uma matriz coluna normalizada. Além disso, pelo mesmo teorema, $1=\left|\lambda_{0}\right|>\left|\lambda_{k}\right|$, para todo $k \geq 1$. Com base nesse teorema, podemos encontrar explicitamente uma expressão $P_{l}$ pela equação (2.4.15) por meio de expansões em autovalores de T. Em geral, não se exige que $T$ seja uma matriz simétrica [7], logo os autovetores de $T$ pela direita não são dados pela transposta dos autovetores da esquerda. A partir de agora, por conveniência, adotaremos a notação bra-ket para o tratamento matricial:

$$
\left\{\begin{array}{l}
T\left|\psi_{k}\right\rangle=\lambda_{k}\left|\psi_{k}\right\rangle \\
\left\langle\phi_{k}\right| T=\left\langle\phi_{k}\right| \lambda_{k}
\end{array}\right.
$$

Onde $\left\{\lambda_{0}, \ldots, \lambda_{n}\right\}$ representa o conjunto de autovalores de $T$. Assumiremos que os autovetores formam um conjunto ortonormalizado:

$$
\left\langle\phi_{i} \mid \psi_{j}\right\rangle=\delta_{i j}
$$

Também adotaremos a propriedade de completeza:

$$
\sum_{k}\left|\psi_{k}\right\rangle\left\langle\phi_{k}\right|=\hat{1}
$$

Levando em conta a teorema de Perron-Fronbenius, existe uma única solução estacionária para a equação (2.4.15) obtida com $\lambda_{0}=1$ e $T\left|\psi_{0}\right\rangle=\left|\psi_{0}\right\rangle$, com $\left|\psi_{0}\right\rangle$ um autovetor não 
degenerado. Com a condição de normalização (2.4.19), existe um $\left\langle\phi_{0}\right|$ tal que:

$$
\left\langle\phi_{0}\right| T=\left\langle\phi_{0}\right|
$$

Podemos escrever $T$ na forma diagonal como:

$$
T=\left(\begin{array}{ccc}
\lambda_{1} & \cdots & 0 \\
\vdots & \ddots & \vdots \\
0 & \cdots & \lambda_{n}
\end{array}\right)
$$

e então:

$$
T^{l}=\left(\begin{array}{ccc}
\lambda_{1}^{l} & \cdots & 0 \\
\vdots & \ddots & \vdots \\
0 & \cdots & \lambda_{n}^{l}
\end{array}\right) .
$$

Considerando a propriedade (2.4.20), podemos escrever:

$$
T^{l}=\sum_{k} \lambda_{k}^{l}\left|\psi_{k}\right\rangle\left\langle\phi_{k}\right|
$$

Pela equação (2.4.15), dado um estado inicial $\left|P_{0}\right\rangle$ e considerando a expressão acima, temos:

$$
\left|P_{l}\right\rangle=\sum_{k} \lambda_{k}^{l}\left|\psi_{k}\right\rangle\left\langle\phi_{k} \mid P_{0}\right\rangle=\left|\psi_{0}\right\rangle\left\langle\phi_{0} \mid P_{0}\right\rangle+\sum_{k \geq 1} \lambda_{k}^{l}\left|\psi_{k}\right\rangle\left\langle\phi_{k} \mid P_{0}\right\rangle
$$

Pelo teorema de Perron-Frobenius, $\left|\lambda_{k}^{l}\right|<1$ para $k \geq 1$, já que $\lambda_{0}=1$. Então, $\lim _{l \rightarrow \infty} \lambda_{k}^{l}=0$. Buscando uma solução estacionária $P_{\text {est }}=\lim _{l \rightarrow \infty}\left|P_{l}\right\rangle$, temos:

$$
\lim _{l \rightarrow \infty}\left|P_{l}\right\rangle=\left|\psi_{0}\right\rangle\left\langle\phi_{0} \mid P_{0}\right\rangle+\sum_{k \geq 1} \lim _{l \rightarrow \infty} \lambda_{k}^{l}\left|\psi_{k}\right\rangle\left\langle\phi_{k} \mid P_{0}\right\rangle=\left|\psi_{0}\right\rangle\left\langle\phi_{0} \mid P_{0}\right\rangle
$$

assim:

$$
\left|P_{\text {est }}\right\rangle=\left|\psi_{0}\right\rangle\left\langle\phi_{0} \mid P_{0}\right\rangle
$$

Um exemplo de aplicação de matrizes estocásticas é um sistema fermiônico binário (ocupação e desocupação) que interage com um reservatório térmico e de partículas com temperatura $T$ e potencial químico $\mu$. Estando a partícula no sistema (ocupado) no instante $l$, ela tem probabilidade $1-p_{l}$ de ir para o reservatório. Estando o sistema desocupado, o reservatório doa uma partícula com probabilidade $p_{l}$. A matrix coluna da probabilidade é dada por:

$$
\left|P_{l}\right\rangle=\left(\begin{array}{c}
1-p_{l} \\
p_{l}
\end{array}\right)
$$


No instante inicial $l=0$ :

$$
\left|P_{0}\right\rangle=\left(\begin{array}{c}
1-p_{0} \\
p_{0}
\end{array}\right)
$$

Considere a matriz estocástica na forma:

$$
T=\left(\begin{array}{cc}
1-\Omega & \alpha \\
\Omega & 1-\alpha
\end{array}\right)
$$

com $0<\Omega<1$ e $0<\alpha<1$. Note que as propriedades (2.4.12) são obedecidas. Os autovalores de $T$ são raízes do polinômio característico:

$$
\operatorname{det}(T-\lambda \hat{1})=0
$$

Como resultado, temos:

$$
\lambda_{0}=1, \lambda_{1}=1-(\alpha+\Omega),
$$

que é coerente com o que estabelece o teorema de Perron-Frobenius: $\lambda_{0}=1$ é correspondente ao estado estacionário e $\left|\lambda_{1}\right|=|1-(\alpha+\omega)|<1$. Tomando como base a normalização (2.4.19), podemos escrever os autovetores:

$$
\begin{gathered}
\left\langle\phi_{0}\left|=\left(\begin{array}{ll}
1 & 1
\end{array}\right),\right| \psi_{0}\right\rangle=\frac{1}{\alpha+\beta}\left(\begin{array}{c}
\alpha \\
\Omega
\end{array}\right), \\
\left\langle\phi_{1}\left|=\left(\begin{array}{ll}
\frac{\Omega}{\alpha+\Omega} & -\frac{\alpha}{\alpha+\Omega}
\end{array}\right),\right| \psi_{1}\right\rangle=\left(\begin{array}{c}
1 \\
-1
\end{array}\right) .
\end{gathered}
$$

Como feito na equação (2.4.25), escrevemos a soluçãoo $\left|P_{l}\right\rangle$ :

$$
\left|P_{l}\right\rangle=\frac{1}{\Omega+\alpha}\left[\left(\begin{array}{l}
\alpha \\
\Omega
\end{array}\right)+[1-(\Omega+\alpha)]^{l}\left(\begin{array}{c}
\Omega-(\Omega+\alpha) P_{0} \\
(\Omega+\alpha) P_{0}-\Omega
\end{array}\right)\right] .
$$

É importante que a soma dos elementos da columa de $\left|P_{l}\right\rangle$ é unitária. Com a expressão acima, encontramos explicitamente o valor para $p_{l}$ (probabilidade de ocupação):

$$
p_{l}=\frac{1}{(\Omega+\alpha)}\left\{\Omega+[1-(\Omega+\alpha)]^{l}\left[(\Omega+\alpha) p_{0}-\Omega\right]\right\}
$$

finalmente, obtemos a solução estacionária:

$$
p_{\text {est }}=\lim _{l \rightarrow \infty} p_{l}=\frac{\Omega}{\Omega+\alpha} .
$$


Fisicamente, na ausência de forças dirigidas, o sistema evolui para o equilíbrio em tempos longos $(l \rightarrow \infty)$. O sistema em questão é análogo a de um fermion em um Ensemble GrandCanônico, cuja a distribuição de equilíbrio é do tipo Fermi-Dirac [8]:

$$
p_{\text {eq }}=\frac{1}{1+e^{\beta(\epsilon-\mu)}},
$$

onde $\beta=\left(k_{B} T\right)^{-1}$ e $\epsilon$ a energia de transição da partícula. Uma vez que nesse caso a solução estacionária (2.4.37) seja a distribuição de equilíbrio, $p_{\text {est }}=p_{\text {eq }}$ e assim, temos a equação:

$$
\frac{\alpha}{\Omega}=e^{\beta(\epsilon-\mu)}
$$

A equação (2.4.39) expressa o balanço detalhado local (BDL). Define-se as taxas de transição entre o reservatório e o sistema de modo que sempre obedeçam o BDL em qualquer instante de tempo. Por outro lado, no caso de sistemas fora de equilíbrio a condição de Balanço Detalhado (BD) (ou balanço detalhado "global"), que expressa a reversibilidade microscópica em sistemas markovianos é violada [10].

Em termos da matriz de transição, a reversibilidade microscópica é descrita a seguir: Considere uma cadeia de Markov com taxas de transições independentes no tempo e um dado processo de transição $n_{0} \rightarrow n_{1} \rightarrow \cdots \rightarrow n_{l}$. Sendo a matriz de transição $T$ deste processo uma matriz regular, existe uma distribuição de probabilidade estacionária e pela equação (2.4.9), escrevemos a probabilidade estacionária, $P\left(n_{0}, n_{1}, \ldots, n_{l}\right)$, da seguinte forma:

$$
P\left(n_{0}, n_{1}, \ldots, n_{l}\right)=T\left(n_{l}, n_{l-1}\right) \ldots T\left(n_{1}, n_{0}\right) P\left(n_{0}\right) .
$$

Tomando o caminho o caminho inverso, temos as transições $n_{l} \rightarrow n_{l-1} \cdots n_{0}$ e podemos escrever a probabilidade $P\left(n_{l}, \ldots, n_{0}\right)$ como:

$$
P\left(n_{l}, \ldots, n_{0}\right)=T\left(n_{0}, n_{1}\right) \ldots T\left(n_{l-1}, n_{l}\right) P\left(n_{l}\right)
$$

podemos escolher uma matriz estocástica auxiliar $\tilde{T}$, obedecendo as propriedades (2.4.12) tal que:

$$
T(n, m) P(m)=\tilde{T}(m, n) P(n),
$$

assim, a expressão para $P\left(n_{l}, \ldots, n_{0}\right)$ pode ser reescrita:

$$
P\left(n_{l}, \ldots, n_{0}\right)=\tilde{T}\left(n_{l}, n_{l-1}\right) \ldots \tilde{T}\left(n_{1}, n_{0}\right) P\left(n_{l}\right)
$$


Em particular, podemos considerar um caso onde:

$$
\tilde{T}(n . m)=T(n . m),
$$

e então, pela expressão (2.4.42):

$$
T(n . m) P(m)=T(m, n) P(n) .
$$

Quando a equação acima é válida, dizemos que $T$ obedece o balanço detalhado. Mais rigorosamente, dada uma matriz estocástica regular $T$ e sua respectiva solução estacionária $P$, $T$ obedece o balanço detalhado se, e somente se, a equação (2.4.45) for válida para todo par $m, n$. Fisicamente falando, em um estado de equilíbrio, espera-se que a probabilidade da transição do estado $m \rightarrow n$ seja a mesma que a probabiliade da transição do estado $n \rightarrow m$. Assim, o balanço detalhado é uma propriedade que depende apenas da matriz estocástica que descreve a cadeia, podendo algumas delas a obedecerem, outras não [9].

\subsubsection{Equação Mestra}

A equação (2.4.11) descreve uma evolução temporal para a matriz probabilidade $P_{l}$. Assumimos nela, um tempo discretizado $l$ que pode ser escrito como $l=t / \tau$, onde $\tau$ é um intervalo de tempo qualquer e a transição temporal $l \rightarrow l+1$ seria equivalente a $t \rightarrow t+\tau$. A partir de agora, faremos uma análise da evolução temporal da probabilidade de uma cadeia de Markov de maneira contínua, tomando $\tau \rightarrow 0$.

Sabemos pela propriedade (2.4.12) que a matriz estocástica é normalizada e podemos escrever:

$$
\sum_{n} T_{n m}=T_{m m}+\sum_{n \neq m} T_{n m}=1
$$

assim, para cada $m$, podemos escrever a diagonal de $T$ por:

$$
T_{m m}=1-\sum_{n \neq m} T_{n m}
$$

Definiremos a matriz de evolução $W$ por:

$$
\left\{\begin{array}{l}
T_{n m}=\tau W_{n m} ; n \neq m, \\
T_{m m}=1-\tau \sum_{n \neq m} W_{n m} .
\end{array}\right.
$$


Na definição acima, os termos da diagonal de $W$ são arbitrários e podemos escolhê-los de modo que dos elementos da coluna seja nulo, isto é $W_{m m}=-\sum_{n \neq m} W_{n m}$. Assim, temos as seguintes propriedades para a matriz de evolução:

$$
\left\{\begin{array}{l}
W_{n m} \geq 0, \text { Para todo } m \neq n, \\
\sum_{n} W_{n m}=0
\end{array}\right.
$$

Pela equação (2.4.11), podemos escrever:

$$
P_{l}(n)=\sum_{m \neq n} T_{n m} P_{l-1}(m)+T_{n n} P_{l-1}(n)
$$

também podemos escrever, pelas relações (2.4.48):

$$
P_{l}(n)=\sum_{m \neq n} T_{n m} P_{l-1}(m)+\left(1-\tau \sum_{m \neq n} W m n\right) P_{l-1}(n)
$$

e após certa álgebra, podemos escrever:

$$
\frac{P_{l}(n)-P_{l-1}(n)}{\tau}=\sum_{m \neq n}\left(W_{n m} P_{l-1}(m)-W_{m n} P_{l-1}\left(n_{0}\right)\right) .
$$

Como já estabelecido, a transição $l-1 \rightarrow l$ equivale à evolução temporal $t-\tau \rightarrow t$. A distribuição $P_{l}(n)$ passa a ser escrita como $P(t, m)$ e tomando o limite de $\tau \rightarrow 0$ temos:

$$
\lim _{\tau \rightarrow 0} \frac{P(t, n)-P(t-\tau, n)}{\tau}=\frac{d P(t, n)}{d t},
$$

assim, reescrevendo $P(t, n)=P_{n}(t)$, a expressão (2.4.52) se torna:

$$
\frac{d P_{n}(t)}{d t}=\sum_{m \neq n}\left(W_{n m} P_{m}(t)-W_{m n} P_{n}(t)\right) .
$$

A relação acima é a equação mestra que descreve a evolução temporal de probabilidade de um dado estado $n$ a partir das taxas de transição $W_{m n}$, que podem ser interpretados como taxas de transição entre o estado $n$ para o $m$. Assim, a variação da probabilidade da ocupação de um estado $n$ no tempo é dada pelo "ganho"de transições $m \rightarrow n$ expresso por $W_{n m} P_{m}$ subtraído pela "perda"de transições de $n \rightarrow m$ expressa por $W_{m n} P_{n}$. Uma série de fenômenos podem ser descritos por uma família de equações similares a essa, como a cinética de reações químicas ou nucleares e processos de transporte em membranas, por exemplo $[1,9]$. Ela ainda pode ser reescrita como:

$$
\frac{d P_{n}(t)}{d t}=\sum_{m} W_{n m} P_{m}(t)
$$


que sugere uma notação matricial dada por:

$$
\dot{P}(t)=W P(t)
$$

onde $P(t)$ é a matriz coluna com os valores das probabilidades $P_{n}(t)$ e $W$ a matriz de evolução. Como feito com a matriz de transição $T$ anteriormente, consideraremos os elementos de $W$ independentes no tempo. A solução dessa equação matricial é dada por:

$$
P(t)=e^{t W} P(0)
$$

onde $P(0)$ é a matriz de probabilidade inicial e $e^{t W}$ é um operador linear dado por:

$$
e^{t W}=\sum_{k=0}^{\infty} \frac{t^{k} W^{k}}{k !}
$$

onde $W^{n}$ é o produto matricial $W \ldots . W$ repetido $n$ vezes e com $W^{0}=\hat{1}$, sendo $\hat{1}$ a matriz identidade.

Assim como foi feito com a matriz de transição $T$, podemos discutir em quais condições é garantida a existência e a unicidade de uma solução estacionária. Passamos agora a estudar uma distribuição $P_{n}^{\text {est }}$ tal que:

$$
\frac{d P_{n}^{\mathrm{est}}}{d t}=0
$$

Fisicamente, esperamos atingir a solução estacionária após longos intervalos de tempo $(t \rightarrow$ $\infty)$. Assim, considere a matriz $P(t)$ solução da equação (2.4.56), a matriz da solução estacionária $P^{\text {est }}$, se existir e for única, é alcançada tomando o limite:

$$
\lim _{t \rightarrow \infty} P(t)=P^{\mathrm{est}}
$$

Analogamente, podemos escrever:

$$
W P^{\mathrm{est}}=0
$$

Pela definição feita em (2.4.48), podemos escrever a matriz de transição como:

$$
T=\hat{1}+\tau W
$$

onde $\tau$ é um intervalo de tempo dado pela relação $t=l \tau$, com 1 sendo um inteiro não negativo. Pela equação (2.4.15), podemos escrever a matriz de probabilidade $P(t)$ como:

$$
P(t)=T^{l} P(0)=T^{t / \tau} P(0)
$$


assim, pela expressão (2.4.62), temos:

$$
P(t)=(\hat{1}+\tau W)^{t / \tau} P(0)
$$

No limite $\tau \rightarrow 0$ :

$$
\lim _{\tau \rightarrow 0}(\hat{1}+\tau W)^{t / \tau}=e^{t W},
$$

que recupera a equação (2.4.56) no caso contínuo. Assim, podemos estudar algumas propriedades da matriz $W$ por meio das propriedades conhecidas da matriz de transição $T$ pela relação (2.4.62). Sendo $T$ regular, pelo teorema de Perron-Frobenius, garantimos a existência e unicidade de uma solução estacionária para equação (2.4.15). Sendo ela $P^{\text {est }}$, sabemos que seu autovalor $\lambda_{0}=1$ é não degenerado e podemos escrever:

$$
T P^{\mathrm{est}}=P^{\mathrm{est}}
$$

Substituindo a expressão (2.4.62), temos:

$$
(1+\tau W) P^{\mathrm{est}}=P^{\mathrm{est}}
$$

que implica em:

$$
W P^{\mathrm{est}}=0
$$

que condiz com a condição $\dot{P^{\text {est }}}=W P^{\text {est }}=0$ para a solução de tempos longos. Assim, podemos enunciar um corolário do teorema de Perron-Frobenius: Seja $T$ uma matriz de transição regular e seja $P^{\text {est }}$ o autovetor (não degenerado) associado ao autovalor $\lambda_{0}=1$, então $P^{\text {est }}$ é também um autovetor não degenerado de $W$, com o autovalor $\lambda=0$. Como já discutido, a matriz $P^{\text {est }}$ tem como entrada números não negativos menores que um. As soluções estacionárias das equações (2.4.15) e (2.4.56) coincidem e podem ser encontradas tanto por $T$ como por $W$. A solução estacionária de ambas as matrizes sempre é obtida para tempos longos e é a mesma independente da condição inicial $P(0)$. Além disso, a parte real dos outros autovalores são menores que zero. Ainda observando a equação (2.4.54), garantimos que $\frac{d P_{n}^{\text {est }}}{d t}=0$ se

$$
W_{n m} P_{m}^{\mathrm{est}}-W_{m n} P_{n}^{\mathrm{est}}=0 .
$$

para todo o par $m$ e $n$, que expressa o balanço detalhado já discutido no contexo de matrizes estocásticas. A distribuição de probabilidade de equilíbrio é a solução estacionária da equação mestra que obedeça o balanço detalhado como expresso acima. 
Podemos repetir o procedimento feito na Seção (2.4.2) com matrizes de transição e encontrar as soluções de $P_{n}(t)$ por meio de uma expansão de autovalores. Então, seja $\left\{\lambda_{0}, \lambda_{1}, \ldots, \lambda_{m}\right\}$ o conjunto finito de autovalores de $W$. Mais uma vez, trataremos um caso geral onde $W$ não é uma matriz simétrica. Usando notação bra-ket, para cada $\lambda_{j}$, podemos escrever:

$$
\left\{\begin{array}{l}
W\left|\psi_{j}\right\rangle=\lambda_{j}\left|\psi_{j}\right\rangle, \\
\left\langle\phi_{j}\right| W=\left\langle\phi_{j}\right| \lambda_{j},
\end{array}\right.
$$

onde são válidas, respectivamente, a ortonormalização e a relação de completeza:

$$
\begin{array}{r}
\left\langle\phi_{n} \mid \psi_{m}\right\rangle=\delta_{n m}, \\
\sum_{j}\left|\psi_{j}\right\rangle\left\langle\phi_{j}\right|=\hat{1} .
\end{array}
$$

Assim, dado a matriz $W$, podemos escrever:

$$
e^{t W}=e^{t W} \hat{1}=e^{t W} \sum_{j}\left|\psi_{j}\right\rangle\left\langle\phi_{j}\left|=\sum_{j} e^{\lambda_{j} t}\right| \psi_{j}\right\rangle\left\langle\phi_{j}\right|,
$$

de acordo com a equação (2.4.57), podemos escrever:

$$
|P(t)\rangle=\sum_{k} e^{t \lambda_{k}}\left|\psi_{k}\right\rangle\left\langle\phi_{k} \mid P_{0}\right\rangle .
$$

Para uma dada matriz de probabilidade $\left|P_{0}\right\rangle$. Tendo $\lambda_{0}=0$, podemos escever:

$$
|P(t)\rangle=\left|\psi_{0}\right\rangle\left\langle\phi_{0} \mid \psi_{0}\right\rangle+\sum_{k \geq 1} e^{\lambda_{k} t}\left|\psi_{k}\right\rangle\left\langle\phi_{k} \mid P_{0}\right\rangle .
$$

Na seção seguinte, discutiremos exemplos com a equação de evolução, obtendo a partir dela, grandezas termodinâmicas como o fluxo de calor e a produção de entropia para sistemas microscópicos.

\subsubsection{Produção de Entropia de Schnakenberg}

Como já discutido na seção (2.1), podemos escrever a variação de entropia do sistema em contato com um reservatório térmico com:

$$
\frac{d S}{d t}=\Pi(t)-\Phi(t)
$$


Para um sistema Markoviano e discretizado, usamos a definição de entropia de Shannon [18]:

$$
S=-k_{\mathrm{B}} \sum_{m} P_{m}(t) \ln P_{m}(t)
$$

cuja variação temporal é dada por:

$$
\frac{d S}{d t}=-k_{\mathrm{B}} \sum_{m} \frac{d P_{m}(t)}{d t} \ln \left(P_{m}(t)\right)-\sum_{m} P_{m} \frac{1}{P_{m}} \frac{d P_{m}}{d t} .
$$

O segundo termo da somatória é nulo. Inserindo a expressão acima na equação mestra, temos Pela equação mestra (2.4.54), temos:

$$
\frac{d S}{d t}=-k_{\mathrm{B}} \sum_{m} \sum_{n \neq m}\left(W_{m n} P_{n}(t)-W_{n m} P_{m}(t)\right) \ln \left(P_{m}(t)\right) .
$$

Definamos a corrente de probabilidade entre estados $n$ e $m$ como $J_{m n}(t):=W_{m n} P_{n}(t)-$ $W_{n m} P_{m}(t)$, de forma que podemos reescrever $\frac{d S}{d t}$ como:

$$
\frac{d S}{d t}=-k_{\mathrm{B}} \sum_{m} \sum_{n \neq m} J_{m n}(t) \ln \left(P_{m}(t)\right) .
$$

Note que a corrente de probabilidade, para cada par $m, n$, tem a seguinte propriedade:

$$
J_{m n}=-J_{n m}, \text { (Anti-simetria) }
$$

Definindo uma função $\gamma_{n}(t)$ qualquer e $\gamma_{n m}(t)=\gamma_{n}(t)-\gamma_{m}(t)$, é possível demonstrar que, sendo válida a propriedade (2.4.81), a seguinte equação é válida:

$$
\sum_{m} \sum_{n \neq m} \gamma_{m}(t) J_{m n}(t)=\frac{1}{2} \sum_{m} \sum_{n \neq m} \gamma_{m n}(t) J_{m n}(t) .
$$

Assim, reescrevemos a expressão (2.1.9) usando a propriedade (2.4.82):

$$
\frac{d S}{d t}=\frac{k_{\mathrm{B}}}{2} \sum_{m} \sum_{n \neq m} J_{m n}(t) \ln \left(\frac{P_{n}(t)}{P_{m}(t)}\right)
$$

A expressão para $\frac{d S}{d t}$ pode ser escrita como:

$$
\begin{aligned}
\frac{d S}{d t} & =\frac{k_{\mathrm{B}}}{2} \sum_{m} \sum_{n \neq m} J_{m n}(t)\left[\ln \left(\frac{P_{n}(t)}{P_{m}(t)}\right)+\ln \left(\frac{W_{n m} W_{m n}}{W_{n m} W_{m n}}\right)\right], \\
& =\frac{k_{\mathrm{B}}}{2} \sum_{m} \sum_{n \neq m} J_{m n}(t) \ln \left(\frac{W_{m n} P_{n}(t)}{W_{n m} P_{m}(t)}\right)+\frac{k_{\mathrm{B}}}{2} \sum_{m} \sum_{n \neq m} J_{m n}(t) \ln \left(\frac{W_{n m}}{W_{m n}}\right) .
\end{aligned}
$$


A primeira deles corresponde à definição da produção de entropia proposta por Schnakemberg [19]. Ela é da forma $(x-y) \ln (x / y)$, que é sempre não negativo para todo $x$ e $y$ reais não nulos. Portanto ela expressa o fato de que a produção de entropia é sempre postiva. Em particular, a expressão é nula se, e somente se, $x=y$. Para a expressão do lado direito, não há restrição em relação a sinais e é denominado "fluxo"de entropia. A expressão (2.4.85), portanto, está na forma da (2.1.17), $\frac{d S}{d t}=\Pi-\Phi$, com:

$$
\begin{aligned}
& \Pi(t)=\frac{k_{\mathrm{B}}}{2} \sum_{m} \sum_{n \neq m} J_{m n}(t) \ln \left(\frac{W_{m n} P_{n}(t)}{W_{n m} P_{m}(t)}\right), \\
& \Phi(t)=-\frac{k_{\mathrm{B}}}{2} \sum_{m} \sum_{n \neq m} J_{m n}(t) \ln \left(\frac{W_{n m}}{W_{m n}}\right) .
\end{aligned}
$$

Como foi discutido na seção (2.1), $\bar{\Pi}$ é apenas nulo no equilíbrio e é escrito na forma (2.1.9), Onde $J$ se identifica com o fluxo e o logaritmo com a força/afinidade. De fato, dado a distribuição de equilíbrio do sistema $P_{m}^{\text {eq }}$ que obedeça a condição de balanço detalhado (2.4.69), $J_{m n}^{\text {eq }}=0$ para todo par $m$ e $n$. Microscopicamente, podemos interpretar que no equilíbrio as correntes entre os estados $n \rightarrow m$ se anulam. Além disso, no estado estacionário, $\frac{d S}{d t}=0$, de forma que $\Pi=0$. Se o estado estacionário for de não equilíbrio (NESS), seque que $\Pi_{\mathrm{NESS}}=\Phi_{\mathrm{NESS}}$ de forma que $\Phi_{\mathrm{NESS}}$ constitui uma forma alternativa para a produção de entropia.

\subsection{Termodinâmica Estocástica para Processos Markovi- anos}

Vamos considerar um processo markoviano descrito pela equação mestra (2.4.54). O sistema é composto por sistemas discretos e não degenerados onde para cada $m$, associamos a uma única energia $\epsilon_{m}$ e ao número de partículas $n_{m}$ pertecentes ao estado $m$. A probabilidade do sistema estar no estado $m$, com energia $\epsilon_{m}$ e com $n_{m}$ partículas em um dado instante $t$ é dado por $P_{m}(t)$, solução da equação mestra (2.4.54). Consideraremos que este sistema interage com um reservatório térmico e de partículas. Suponhamos que esta interação possa ser controlada externamente por meio de um parâmetro de ajuste $k$ e por simplicidade, consideraremos que essa variação externa dê origem a uma força que realize trabalho no sistema. Fixo um estado $m$, o parâmetro $k$ altera, sem dissipações, a energia $\epsilon_{m}$ [18]. 
Podemos construir um sistema que evolui no tempo para regiões fora do equilíbrio considerando que o parâmetro externo $k=k(t)$ varie no tempo. Podemos escrever, para um dado estado acessível $m$, a energia como uma função de $k, \epsilon_{m}(k)$ e a sua diferencial no tempo é dada por:

$$
\dot{\epsilon}_{m}=\dot{k} \frac{d \epsilon_{m}}{d k} .
$$

A energia interna e o número de partículas são dadas por:

$$
\begin{aligned}
& U=\sum_{m} P_{m}(t) \epsilon_{m}(t), \\
& N=\sum_{m} P_{m}(t) n_{m},
\end{aligned}
$$

onde assumiremos que o número de partículas em cada estado $m, n_{m}$, seja fixo no tempo. Suas respectivas diferenciais no tempo são dadas por:

$$
\begin{aligned}
& \dot{U}=\sum_{m}\left(P_{m}(t) \dot{\epsilon}_{n}(t)\right)+\sum_{m}\left(\epsilon_{n}(t) \frac{d P_{n}(t)}{d t}\right), \\
& \dot{N}=\sum_{m}\left(\frac{d P_{m}(t)}{d t} n_{m}\right) .
\end{aligned}
$$

Pela primeira lei da Termodinâmica [6,7]:

$$
\dot{U}(t)=\dot{W}(t)+\dot{Q}(t)+\dot{W}_{\text {chem }}
$$

onde $W(t)$ é o trabalho exercido pelo protocolo externo $k, Q(t)$ é o calor trocado entre o sistema e o reservatório e $W_{\text {chem }}$ o trabalho químico associado a troca de partículas entre o sistema e o reservatório. Comparando as derivadas no tempo, escrevemos:

$$
\dot{W}(t)+\dot{Q}(t)+\dot{W}_{\text {chem }}=\sum_{m}\left(P_{m}(t) \dot{\epsilon}_{m}(t)\right)+\sum_{m}\left(\epsilon_{m}(t) \frac{d P_{m}(t)}{d t}\right) .
$$

Como discutido acima e com base na equação (2.5.1), toda a variação no tempo da energia $\epsilon_{m}$ para um estado fixo $m$ é devido ao trabalho realizado pelo protocolo externo $k$, assim, podemos escrever:

$$
\begin{aligned}
\dot{W}(t) & =\sum_{m}\left(P_{m}(t) \dot{\epsilon}_{m}(t)\right) \\
\dot{Q}(t) & =\sum_{m}\left(\epsilon_{m}(t) \frac{d P_{m}(t)}{d t}\right)-\dot{W}_{\text {chem }}
\end{aligned}
$$


Além disso, podemos escrever:

$$
\dot{W}_{\text {chem }}(t)=\mu \dot{N}
$$

onde $\dot{N}$ é dado pela equação (2.5.5). Portanto, pela equação (2.5.5), podemos escrever:

$$
\dot{Q}(t)=\sum_{m} \frac{d P_{m}(t)}{d t}\left(\epsilon_{m}(t)-\mu n_{m}\right)
$$

De acordo com a equação mestra, podemos relacionar $\dot{Q}(t)$ com a corrente $J_{m n}$ :

$$
\dot{Q}(t)=\sum_{m} \sum_{n \neq m}\left(W_{m n} P_{n}(t)-W_{n m} P_{m}(t)\right)\left(\epsilon_{m}(t)-\mu n_{m}\right) .
$$

Definindo $\gamma_{m}(t)=\epsilon_{m}(t)-\mu n_{m}, \gamma_{m n}(t):=\gamma_{m}(t)-\gamma_{n}(t)$ e adotando a corrente de probabilidade $J_{m n}$, podemos aplicar mais uma vez a propriedade (2.4.82) e reescrever a expressão (2.5.12) como:

$$
\dot{Q}(t)=\sum_{m} \sum_{n \neq m} J_{m n}(t)\left(\epsilon_{m}(t)-\mu n_{m}\right),
$$

assim, o fluxo de calor $\dot{Q}(t)$ pode ser reescrito como:

$$
\dot{Q}(t)=\frac{1}{2} \sum_{m} \sum_{n \neq m}\left(\epsilon_{m}-\mu n_{m}\right) J_{m n}(t),
$$

De forma análoga, o trabalho químico $W_{\text {chem }}$ é dado por:

$$
W_{\text {chem }}^{\cdot}=\frac{1}{2} \sum_{m} \sum_{n \neq m} \mu n_{m} J_{m n}(t)
$$

\subsection{Equações de Fokker-Planck}

Em 1828, o botânico escocês Robert Brown observou um movimento irregular (aleatório) de pólens suspensos em água. Tal fenômeno, posteriormente, passou a ser chamado de Movimento Browniano e foi amplamente estudado por cientistas no século XX. Iniciando por Einstein em 1905, passando por Perrin (1908), que usou o fenômeno para estimar o número de Avogadro e, assim, provou a teoria cinética da matéria. No mesmo ano, o físico francês Paul Langevin conseguiu descrever o fenômeno por meio de uma equação estocástica (equação de Langevin), encontrado os valores exatos para as médias da posição e da velocidade de uma partícula realizando o movimento Browniano. Sendo a solução de uma equação estocástica, a velocidade da partícula é uma função aleatória e a sua distribuição de probabilidade 
pode ser obtida por meio de uma equação de Fokker-Planck [9]. Nesta seção, discutiremos esses resultados e os usaremos para estudar a termodinâmica estocástica de uma partícula Browniana que interage com um reservatório térmico.

\subsubsection{Equações de Langevin}

Considere uma partícula de massa $m$ suspensa em um dado fluido. De acordo com Langevin [20], as moléculas que o compoem colidem com o corpo suspenso e devido ao comportamento térmico, esses choques são aleatórios, gerando o movimento irregular da partícula. Além disso, uma força de arrasto com o coeficiente $\alpha$ é exercido sobre a partícula. Dessa forma, escrevemos a equação de movimento da partícula, para uma dimensão, como:

$$
m \dot{v}=-\alpha v+F_{a}(t),
$$

onde $\alpha$ é o coeficiente de arrasto do fluido e $F_{a}(t)$ é uma força aleatória devido a colisões da partícula com as moléculas do fluido. Consideramos que as forças aleatórias obedecem as seguintes condições em relação a suas médias:

$$
\begin{aligned}
& \left\langle F_{a}(t)\right\rangle=0, \\
& \left\langle F_{a}(t) F_{a}\left(t^{\prime}\right)\right\rangle=B \delta\left(t-t^{\prime}\right) .
\end{aligned}
$$

A equação (2.6.2) estabelece que, em média, a força aleatória é nula. Dessa maneira, não há uma direção ou sentido preferencial para ela. A relação (2.6.3) estabelece que colisões em instantes diferentes são independentes. O termo $B$ depende da temperatura do fluido e será encontrado a seguir. Reescrevemos a equação (2.6.1) da seguinte forma:

$$
\dot{v}=-\gamma v+\zeta(t),
$$

onde a função $\zeta(t)$ é um ruido branco, obedencendo as seguintes condições:

$$
\begin{aligned}
& \langle\zeta(t)\rangle=0, \\
& \left\langle\zeta(t) \zeta\left(t^{\prime}\right)\right\rangle=\Gamma \delta\left(t-t^{\prime}\right),
\end{aligned}
$$

onde: $\gamma=\alpha / m, \zeta(t)=F_{a}(t) / m$ e $\Gamma=B / m^{2}$. A solução da equação (2.6.4) é dada por:

$$
v(t)=v_{0} e^{-\gamma t}+\int_{o}^{t} \zeta\left(t^{\prime}\right) e^{\gamma\left(t^{\prime}-t\right)} d t^{\prime} .
$$


Dado que $\dot{x}=v(t)$, podemos escrever:

$$
x(t)=x_{0}+\frac{v_{0}}{\gamma}\left(1-e^{-\gamma t}\right)+\int_{0}^{t}\left(\int_{0}^{t^{\prime}} \zeta\left(t^{\prime \prime}\right) e^{\gamma t^{\prime \prime}} d t^{\prime \prime}\right) e^{\gamma t^{\prime}} d t^{\prime}
$$

que resulta em:

$$
x(t)=x_{0}+\frac{v_{0}}{\gamma}\left(1-e^{-\gamma t}\right)+\frac{1}{\gamma} \int_{0}^{t} \zeta\left(t^{\prime \prime}\right)\left(1-e^{\gamma\left(t^{\prime \prime}-t\right)}\right) d t^{\prime \prime} .
$$

Tomando os valores médios das expressões para a posição e a velocidade, usando a condição (2.6.2), podemos escrever:

$$
\begin{aligned}
& \langle x(t)\rangle=x_{0}+\frac{v_{0}}{\gamma}\left(1-e^{-\gamma t}\right), \\
& \langle v(t)\rangle=v_{0} e^{-\gamma t}
\end{aligned}
$$

de modo análogo, podemos obter a média da velocidade quadrática $\left\langle v^{2}(t)\right\rangle$ :

$$
\left\langle v^{2}(t)\right\rangle=\left\langle v_{0}^{2} e^{-2 \gamma t}+\int_{0}^{t} \int_{0}^{t^{\prime}} \zeta\left(t^{\prime}\right) \zeta\left(t^{\prime \prime}\right) e^{\gamma\left(t-t^{\prime}\right)} e^{\gamma\left(t-t^{\prime \prime}\right)} d t^{\prime} d t^{\prime \prime}+2 v_{0} e^{-\gamma t} \int_{0}^{t} \zeta\left(t^{\prime}\right) e^{\gamma\left(t-t^{\prime}\right)} d t^{\prime}\right\rangle
$$

Mais uma vez, usando as propriedades (2.6.2) e (2.6.3), reescrevemos a expressão acima:

$$
\left\langle v^{2}(t)\right\rangle=v_{0}^{2} e^{-2 \gamma t}+\frac{\Gamma}{2 \gamma}\left(1-e^{-2 \gamma t}\right)
$$

como expresso na equação $(2.6 .11),\langle v(t)\rangle^{2}=v_{0}^{2} e^{-2 \gamma t}$. Usando a relação acima, escrevemos a variância da velocidade dada por:

$$
b(t)=\left\langle v^{2}(t)\right\rangle-\langle v(t)\rangle^{2}=\frac{\Gamma}{2 \gamma}\left(1-e^{-2 \gamma t}\right) .
$$

Para tempos longos, $\langle v(t)\rangle \rightarrow 0$ e nesse limite, a velocidade quadrática média pode ser escrita como:

$$
\left\langle v^{2}\right\rangle=\frac{\Gamma}{2 \gamma}
$$

Pelo Teorema da Equipartição de Energia [9], podemos escrever, para um grau de liberdade: $(1 / 2) m\left\langle v^{2}\right\rangle=(1 / 2) k_{\mathrm{B}} T$. Tomando a relação (2.6.15), uma expressão explícita para $\Gamma$ é encontrada:

$$
\Gamma=\frac{2 k_{\mathrm{B}} \gamma T}{m} .
$$

O valor $\Gamma$ é chamado de temperatura reduzida. 
A equação de Langevin (2.6.4) é uma equação estocástica e a sua solução, $v(t)$, também varia aleatoriamente no tempo. Já conhecemos o seu valor médio pela expressão (2.6.11) e podemos considrerar sua distribuição de probabilidade $P=P(v, t)$, que é solução da equação de Fokker-Planck (2.6.17) [9], que pode ser escrita da seguinte forma:

$$
\frac{\partial P}{\partial t}=-\frac{\partial J(v, t)}{\partial v}
$$

onde $J(v, t)$ é a corrente de probabilidade, dada por:

$$
J(v, t)=-\gamma v P(v, t)-\frac{\Gamma}{2} \frac{\partial}{\partial v} P(v, t) .
$$

Uma dedução detalhada da equação (2.6.17) pode ser encontrado em [9]. Estando em contato com um reservatório térmico de temperatura $T$, a equação (2.6.17) tem como solução estacionária consistente com a distribuição de Maxwell-Boltzmann:

$$
P_{\mathrm{est}}=\frac{1}{Z} e^{-\beta m v^{2} / 2}
$$

onde $Z$ é a função de partição do sistema e $\beta=\left(k_{\mathrm{B}} T\right)^{-1}$. A probabilidade $P(v, t)$ é normalizada:

$$
\int_{-\infty}^{\infty} P(v, t) d v=1, \text { para todo tempo t }
$$

onde a integral acima se estende por todo o espaço de velocidades.

\subsubsection{Termodinâmica Estocástica para Partículas Brownianas}

Considere uma partícula de massa $m$, descrita por uma equação de Langevin (2.6.4). O sistema interage com um reservatório térmico de temperatura $T$. Escreveremos a entropia do sistema como uma forma contínua da expressão (2.4.77):

$$
S(t)=-k_{\mathrm{B}} \int_{-\infty}^{\infty} P(v, t) \ln [P(v, t)] d v,
$$

considerando uma variação da entropia ao longo do tempo, calculamos sua derivada:

$$
\frac{d S}{d t}=-\int_{-\infty}^{\infty} \frac{\partial}{\partial t}(P(v, t) \ln [P(v, t)])=-\int_{-\infty}^{\infty} \frac{\partial P}{\partial t} \ln [P(v, t)] d v .
$$

Pela equação de Fokker-Planck, podemos escrever:

$$
\frac{d S}{d t}=\int_{-\infty}^{\infty} \frac{\partial J}{\partial v} \ln [P(v, t)] d v
$$


Integrando por partes a expressão acima, temos:

$$
\frac{d S}{d t}=\left.J \ln [P(v, t)]\right|_{-\infty} ^{\infty}-\int_{-\infty}^{\infty} J \frac{\partial}{\partial v} \ln [P(v, t)] d v
$$

onde assumimos que $P(v, t)$ e $J(v, t)$ se anulam nas extremidades. Dessa forma,

$$
\frac{d S}{d t}=-\int_{-\infty}^{\infty} J \frac{\partial}{\partial v} \ln [P(v, t)] d v .
$$

Pela expressão (2.6.18), podemos escrever:

$$
\frac{\partial \ln [P(v, t)]}{\partial v}=\frac{1}{P(v, t)} \frac{\partial}{\partial v} P(v, t)=-\frac{2 \gamma v}{\Gamma}-\frac{2 J(v, t)}{\Gamma P(v, t)}
$$

Retornando para a expressão (2.6.25), temos:

$$
\frac{d S}{d t}=\int_{-\infty}^{\infty} \frac{2 J^{2}}{\Gamma P} d v+\int \frac{\gamma v}{\Gamma} J d v
$$

onde expressão acima tem novamente a forma $\frac{d S}{d t}=\Pi-\Phi$ :

$$
\begin{aligned}
& \Pi=\int_{-\infty}^{\infty} \frac{2 J^{2}}{\Gamma P} d v \geq 0, \\
& \Phi=-\int_{-\infty}^{\infty} \frac{\gamma v}{\Gamma} J d v
\end{aligned}
$$

Assim como no caso anterior, $\Pi \geq 0$ e mostraremos adiante que no caso de um estado estacionário de não equilíbrio, $\Pi_{\mathrm{NESS}}=\Phi_{\text {Ness }}$. Para um caso onde o sistema evoluiu para o equilíbrio, as expressões acima se anulam, como esperado, tendo $\Pi=\Phi=0$.

Em relação a energia interna do sistema $U$, sabemos que $U=\langle E\rangle$, onde no presente caso, $E=(1 / 2) m v^{2}$. De forma análoga ao caso de processos markovianos, calculamos a variação da energia interna considerando sua interação com o reservatório:

$$
\dot{U}=\int_{-\infty}^{\infty} \frac{d}{d t}[E P(v, t)]=\frac{m}{2} \int_{-\infty}^{\infty} v^{2} \frac{\partial P}{\partial t} d v .
$$

De acordo com a equação de Fokker-Planck (2.6.17), temos então:

$$
\dot{U}=-\frac{m}{2} \int_{-\infty}^{\infty} v^{2} \frac{\partial J}{\partial v} d v
$$

Considerando também a definição de corrente de probabilidade dada por (2.6.18), reescrevemos a expressão acima como:

$$
\dot{U}=\frac{m \gamma}{2}\left(\int_{-\infty}^{\infty} v^{2} \frac{\partial}{\partial v}(v P) d v+\frac{\Gamma}{2 \gamma} \int_{-\infty}^{\infty} v^{2} \frac{\partial^{2} P}{\partial v^{2}} d v\right),
$$


onde a integral da esquerda pode ser calculada usando integração por partes, considerando mais uma vez que $P(v, t)$ se anula nas extremidades:

$$
\int_{-\infty}^{\infty} v^{2} \frac{\partial}{\partial v}(v P) d v=-2\left\langle v^{2}\right\rangle
$$

Para a integral do lado direito, o método é repetido, considerando agora que $\frac{\partial P}{\partial v} \rightarrow 0$ nos limites $v \rightarrow \pm \infty$ :

$$
\frac{\Gamma}{2 \gamma} \int_{-\infty}^{\infty} v^{2} \frac{\partial^{2} P}{\partial v^{2}} d v=\frac{\Gamma}{\gamma}
$$

Portanto, na ausência de forças externas a equação (2.6.32) assume uma forma similar a da primeira lei da termodinâmica:

$$
\dot{U}=-\dot{Q}
$$

onde o fluxo de calor $\dot{Q}$ é dado por:

$$
\dot{Q}=m \gamma\left(\left\langle v^{2}\right\rangle-\frac{\Gamma}{2 \gamma}\right)
$$

Considere agora um sistema em uma dimensão com uma força externa não dissipativa dependente do tempo $F(t)$. A equação de Langevin (2.6.4) torna-se:

$$
\dot{v}=-\gamma v+f(t)+\zeta(t)
$$

$\operatorname{com} f(t)=F(t) / m$. A equação de Fokker-Planck associada é dada novamente por:

$$
\frac{\partial P}{\partial t}=-\frac{\partial J}{\partial v}-\frac{\partial(f(t) P)}{\partial v}
$$

A variação de energia interna é dada por:

$$
\dot{U}=\frac{d\langle E\rangle}{d t}
$$

Como fizemos anteriormente, a energia é dada por:

$$
\dot{U}=\langle\dot{E}\rangle=-\frac{m}{2} \int_{-\infty}^{\infty} v^{2}\left(\frac{\partial J}{\partial v}+f(t) \frac{\partial P}{\partial v}\right) d v
$$

e recalculando cada termo acima temos que:

$$
-\frac{m}{2} \int_{-\infty}^{\infty} v^{2} f \frac{\partial P}{\partial v} d v=m f(t)\langle v\rangle
$$

assim, temos a expressão $\dot{U}=-(\dot{W}+\dot{Q})$, onde:

$$
\begin{aligned}
\dot{W} & =-m f(t)\langle v\rangle, \\
\dot{Q} & =m \gamma\left(\left\langle v^{2}\right\rangle-\frac{\Gamma}{2 \gamma}\right) .
\end{aligned}
$$


de forma que a evolução temporal da energia interna tem uma forma consistente com a primeira lei da termodinâmica e o fluxo de trabalho (potência) se deve à força externa. Tomando a definição de fluxo de entropia dada por (2.6.29), podemos reescrevê-la em termos do fluxo de calor $\dot{Q}$, efetuando a integral da expressão (2.6.29):

$$
\Phi=\frac{\dot{Q}}{T}
$$

Para encontrar os valores médios de $v(t)$ e $x(t)$, precisamos encontrar explicitamente a solução geral da equação (2.6.37). Para resolvê-las, adotaremos métodos análogos ao aplicado na equação (2.6.1) e, considerando as condições de contorno $x(0)=x_{0}$ e $v(0)=v_{0}$, encontramos:

$$
v(t)=v_{0} e^{-\gamma t}+\int_{0}^{t} e^{\gamma\left(t^{\prime}-t\right)}\left(f\left(t^{\prime}\right)+\zeta\left(t^{\prime}\right)\right) d t^{\prime}
$$

e:

$$
x(t)=x_{0}+\frac{v_{0}}{\gamma}\left(1-e^{-\gamma t}\right)+\int_{0}^{t}\left[\int_{0}^{t^{\prime}} e^{\gamma\left(t^{\prime \prime}-t^{\prime}\right)}\left(f\left(t^{\prime \prime}\right)+\zeta\left(t^{\prime \prime}\right)\right) d t^{\prime \prime}\right] d t^{\prime} .
$$

Em média, obtemos:

$$
\begin{gathered}
\langle v(t)\rangle=v_{0} e^{-\gamma t}+\int_{0}^{t} e^{-\gamma\left(t^{\prime}-t\right)} f\left(t^{\prime}\right) d t^{\prime} \\
\langle x(t)\rangle=x_{0}+\frac{v_{0}}{\gamma}\left(1-e^{-\gamma t}\right)+\int_{0}^{t}\left[e^{\gamma\left(t^{\prime \prime}-t^{\prime}\right)} f\left(t^{\prime \prime}\right) d t^{\prime \prime}\right] d t^{\prime} .
\end{gathered}
$$

Considerando a equação de Langevin, a equação de Fokker-Planck e definição de variância (2.6.14), podemos escrever a evolução temporal da média e da variância da velocidade por:

$$
\begin{gathered}
\frac{d\langle v(t)\rangle}{d t}=-\gamma\langle v(t)\rangle+f(t), \\
\frac{d b(t)}{d t}=-2 \gamma b(t)+\Gamma
\end{gathered}
$$

A seguir, aplicaremos esses conceitos em modelos de máquinas colisionais em escala mesoscópica. 


\section{Capítulo 3}

\section{Modelo Colisional para um Sistema de Quantum Dot}

A performance de máquinas térmicas em máxima potência tem sido amplamente estudada na literatura $[13,16,17,21,23-26]$ e constitui um dos principais desafios da termodinâmica de não equilíbrio, tanto em sistemas clássicos quanto em quânticos. Neste capítulo estudaremos uma máquina térmica colisional composta por um quantum-dot (QD) que interage com diferentes reservatórios térmicos e de partículas. Considerando a diferença de temperatura e a interação reservatório-partícula, haverá extração de trabalho, trabalho químico e calor trocado. Conforme exemplificado na Seção (2.4.2), esse sistema é descrito por uma cadeia de Markov e no equilíbrio, a probabilidade é dada pela distribuição de Fermi-Dirac. Fora desse regime, será solução da equação mestra (2.4.54). Aplicaremos os resultados obtidos na Seção (2.5) para estudar o modelo colisional dessa interação termodinâmica.

Modelos colisionais para máquinas térmicas consistem em sistemas que interagem sequecialmente com mais de um reservatório, sendo a transição de um para o outro instantânea, em geral causada por uma perturbação externa. Eles têm sido descritos como versões mais apropriadas e realistas para estudar a performance de máquinas em alguns casos [26] em que o processo abrupto "simularia"um processo adiabático. Em outros casos, eles descreveriam diferentes interações (mais fracas em alguns casos) entre diferentes partes do sistema e do reservatório. Na mecânica quântica, eles fariam o papel de diferentes interações descor- 
relacionadas entre a partícula e o reservatório. Por simplicidade, consideraremos um caso particular de dois reservatórios térmicos que interagem ciclicamente com um sistema de QD. Similar ao exemplo discutido na Seção (2.4.2), consideraremos um sistema fermiônico que interage com um reservatório térmico e de partículas. O reservatório pode doar ou receber partículas do sistema. Por simplicidade, apenas um fermion pode ocupar o sistema por vez e estando ele ocupado, o reservatório pode receber a partícula do sistema com uma dada probabilidade em um dado instante $t$. Inicialmente, esse sistema irá interagir por um dado intervalo de tempo com um reservatório quente e, abruptamente, entrará em contato com um reservatório térmico frio, voltando para o primeiro reservatório após um período completo, formando assim um ciclo termodinâmico. A perturbação que causa a mudança de reservatório impede que o sistema evolua para um estado estacionário de equilíbrio, porém a equação mestra que a descreve tem uma solução estacionária (de não equilíbrio), alcançada para vários ciclos. Esse regime é chamado de Non-Equilibrium Steady State ou NESS e, ao contrário do estado estacionário de equilíbrio, produz entropia continuamente. Espera-se extrair desse ciclo trabalho útil dependendo da escolha apropriada dos parâmetros. Conforme discutido na Seção (2.3), tal abordagem seria mais realista para máquinas térmicas endoreversíveis, onde os processos termodinâmicos que o compoem não são quasi-estáticos. Tal modelo foi amplamente estudado nas referências [21,22] para o caso de tempos simétricos, isto é, o sistema (quantum dot) fica em contato metade do período com o reservatório quente e a outra metade no quente. Aqui introduziremos a ideia de tempos assimétricos na interação entre o sistema e reservatórios como uma forma de otimizar a performance da máquina. Em particular, estudaremos a influência da assimetria no tempo entre as interações térmicas na potência e na eficiência da máquina, ambas sendo calculadas com os resultados da seção (2.5). Os resultados desta pesquisa foram publicados em forma de artigo na revista Phisical Review Research, disponível na íntegra na seção (A) do apêndice.

\subsection{Modelo e Solução Exata}

O sistema fermiônico interage com dois reservatórios sequencialmente com temperaturas $T_{\mathrm{C}}$ e $T_{\mathrm{H}}$ e potenciais químicos $\mu_{\mathrm{C}}$ e $\mu_{\mathrm{H}}$, sendo respectivamente as temperaturas e os potenciais químicos dos reservatórios frio e quente. A interação entre o sistema e os reservatórios se 
dá de maneira cíclica com periodo $\tau$. O sistem interage com o reservatório quente durante um intervalo de tempo $\tau_{\mathrm{H}}$ e interage com um reservatório frio durante o intervalo de tempo $\tau-\tau_{H}$. Por simplicidade, a mudança entre reservatórios é assumida instantânea (intervalo de tempo próximo de zero). Este processo é considerado adiabático.

A interação termodinâmica entre o sistema e o reservatório pode ser modelado por meio de uma cadeia de Markov. Para cada reservatório $i=\{\mathrm{H}, \mathrm{C}\}$, a taxa de transição de partículas entre o reservatório para o sistema é dada por $\omega_{i}$. Estando a partícula ocupando o sistema, a taxa de transição para o reservatório é dado por $\bar{\omega}_{i}$. A matriz de evolução que descreve esse sistema é dada por:

$$
W_{i}=\left(\begin{array}{cc}
-\omega_{i} & \bar{\omega}_{i} \\
\omega_{i} & -\bar{\omega}_{i}
\end{array}\right),
$$

assim, a equação mestra para cada reservatório $i$ é dada por:

$$
\dot{P}_{i}(t)=W_{i} P_{i}(0)
$$

A solução da equação mestra pode ser ser encontrada de acordo com os métodos descritos na seção (2.4.3). Para cada reservatório $i$, escrevemos:

$$
P_{i}(t)=\left(\begin{array}{c}
1-p_{i}(t) \\
p_{i}(t)
\end{array}\right),
$$

onde $p_{i}(t)$ é a probabilidade de ocupação do sistema quando está em contato com o reservatório $i$. Para cada reservatório $i$, escrevemos a solução geral da equaçao (3.1.3), após uma solução transiente, como:

$$
\begin{aligned}
& p_{\mathrm{H}}(t)=\frac{\omega_{\mathrm{H}}}{\omega_{\mathrm{H}}+\bar{\omega}_{\mathrm{H}}}+\left[p_{\mathrm{H}}(0)-\frac{\omega_{\mathrm{H}}}{\bar{\omega}_{\mathrm{H}}+\omega_{\mathrm{H}}}\right] e^{-\left(\omega_{\mathrm{H}}+\bar{\omega}_{\mathrm{H}}\right) t} \\
& p_{\mathrm{C}}(t)=\frac{\omega_{\mathrm{C}}}{\bar{\omega}_{\mathrm{C}}+\omega_{\mathrm{C}}}+\left[p_{\mathrm{C}}\left(\tau_{\mathrm{H}}\right)-\frac{\omega_{\mathrm{C}}}{\omega_{\mathrm{C}}+\bar{\omega}_{\mathrm{C}}}\right] e^{-\left(\omega_{\mathrm{C}}+\bar{\omega}_{\mathrm{C}}\right)\left(t-\tau_{\mathrm{H}}\right)}
\end{aligned}
$$

A expressão da probabilidade deve ser contínua (mesmo com a mudança instantânea de reservatórios), assim, é necessário estabelecer que entre as transições, $p_{\mathrm{C}}\left(\tau_{\mathrm{H}}\right)=p_{\mathrm{H}}\left(\tau_{\mathrm{H}}\right)$. Uma vez que o sistema retorna ao estado inicial após um período, segue que $p_{\mathrm{C}}(\tau)=p_{\mathrm{H}}(0)$. Aplicando as condições de contorno explicitamente nas expressões $(3.1 .4,3.1 .5)$, encontramos:

$p_{\mathrm{H}}(t)=\frac{\omega_{\mathrm{H}}}{\omega_{\mathrm{H}}+\bar{\omega}_{\mathrm{H}}}-\frac{e^{-\left(\omega_{\mathrm{H}}+\bar{\omega}_{\mathrm{H}}\right) t}\left[1-e^{-\left(\omega_{\mathrm{C}}+\bar{\omega}_{\mathrm{C}}\right)\left(\tau-\tau_{\mathrm{H}}\right)}\right]}{1-e^{-\left(\omega_{\mathrm{H}}+\bar{\omega}_{\mathrm{H}}\right) \tau_{\mathrm{H}}-\left(\omega_{\mathrm{C}}+\bar{\omega}_{\mathrm{C}}\right)\left(\tau-\tau_{\mathrm{H}}\right)}} \frac{\omega_{\mathrm{H}} \bar{\omega}_{\mathrm{C}}-\bar{\omega}_{\mathrm{H}} \omega_{\mathrm{C}}}{\left(\omega_{\mathrm{H}}+\bar{\omega}_{\mathrm{H}}\right)\left(\omega_{\mathrm{C}}+\bar{\omega}_{\mathrm{C}}\right)}, t=\left[0, \tau_{\mathrm{H}}\right](\bmod \tau)$, 
$p_{\mathrm{C}}(t)=\frac{\omega_{\mathrm{C}}}{\omega_{\mathrm{C}}+\bar{\omega}_{\mathrm{C}}}+\frac{e^{-\left(\omega_{\mathrm{C}}+\bar{\omega}_{\mathrm{C}}\right)\left(t-\tau_{\mathrm{H}}\right)}\left[1-e^{-\left(\omega_{\mathrm{H}}+\bar{\omega}_{\mathrm{H}}\right) \tau_{\mathrm{H}}}\right]}{1-e^{-\left(\omega_{\mathrm{H}}+\bar{\omega}_{\mathrm{H}}\right) \tau_{\mathrm{H}}-\left(\omega_{\mathrm{C}}+\bar{\omega}_{\mathrm{C}}\right)\left(\tau-\tau_{\mathrm{H}}\right)}} \frac{\omega_{\mathrm{H}} \bar{\omega}_{\mathrm{C}}-\bar{\omega}_{\mathrm{H}} \omega_{\mathrm{C}}}{\left(\omega_{\mathrm{H}}+\bar{\omega}_{\mathrm{H}}\right)\left(\omega_{\mathrm{C}}+\bar{\omega}_{\mathrm{C}}\right)}, t=\left[\tau_{\mathrm{H}}, \tau\right]\left(\bmod \tau_{\mathrm{H}}\right)$.

As expressões (3.1.7) recuperam os resultados obtidos pelas referências [21,22] para $\tau_{\mathrm{H}}=\tau / 2$. Podemos escrever a corrente de probabilidade conforme descrita na seção (2.4.4) em termos de $\omega_{i}$ e $\bar{\omega}_{i}$. Assim, para cada reservatório $i$, a corrente de probabilidade $J_{i}(t)$ é dada por:

$$
J(t)=\left\{\begin{array}{l}
J_{\mathrm{H}}(t)=\omega_{\mathrm{H}}\left(1-p_{\mathrm{H}}(t)\right)-\bar{\omega}_{\mathrm{H}} p_{\mathrm{H}}(t), t=\left[0, \tau_{\mathrm{H}}\right](\bmod \tau), \\
J_{\mathrm{C}}(t)=\omega_{\mathrm{C}}\left(1-p_{\mathrm{C}}(t)\right)-\bar{\omega}_{\mathrm{C}} p_{\mathrm{C}}(t), t=\left[\tau_{\mathrm{H}}, \tau\right](\bmod \tau) .
\end{array}\right.
$$

É conveniente calcularmos as médias sobre o período de oscilação, de forma que podemos encontrar o valor médio da corrente de probabilidade ao longo de um ciclo $\tau$ :

$$
\bar{J}=\frac{1}{\tau} \int_{0}^{\tau} J(t) d t=\frac{1}{\tau}\left(\int_{0}^{\tau_{\mathrm{H}}} J_{\mathrm{H}}(t) d t+\int_{\tau_{\mathrm{H}}}^{\tau} J_{\mathrm{C}}(t) d t\right) .
$$

que pode ser reescrito como:

$$
\bar{J}=\overline{J_{\mathrm{H}}}+\overline{J_{\mathrm{C}}}
$$

com:

$$
\begin{aligned}
& \bar{J}_{\mathrm{H}}=\frac{1}{\tau}\left(\int_{0}^{\tau_{\mathrm{H}}} J_{\mathrm{H}}(t) d t\right) \\
& \bar{J}_{\mathrm{C}}=\frac{1}{\tau}\left(\int_{\tau_{\mathrm{H}}}^{\tau} J_{\mathrm{C}}(t) d t\right)
\end{aligned}
$$

Podemos encontrar explicitamente o valor de $\bar{J}$ usando a equação mestra na forma (2.4.54), que pode ser reescrita como:

$$
\dot{p}(t)=\left\{\begin{array}{l}
\left.\dot{p}_{\mathrm{H}}(t)=\omega_{\mathrm{H}}\left(1-p_{\mathrm{H}}\right)\right)-\bar{\omega}_{\mathrm{H}} p_{\mathrm{H}}(t)=J_{\mathrm{H}}(t) ; t \in\left[0, \tau_{\mathrm{H}}\right], \\
\left.\dot{p}_{\mathrm{C}}(t)=\omega_{\mathrm{H}}\left(1-p_{\mathrm{C}}\right)\right)-\bar{\omega}_{\mathrm{H}} p_{\mathrm{C}}(t)=J_{\mathrm{C}}(t) ; t \in\left[\tau_{\mathrm{H}}, \tau\right] .
\end{array}\right.
$$

Note que:

$$
\bar{J}_{\mathrm{H}}+\bar{J}_{\mathrm{C}}=\frac{1}{\tau}\left(\int_{0}^{\tau_{\mathrm{H}}} \dot{p}_{\mathrm{H}}(t) d t+\int_{\tau_{\mathrm{H}}}^{\tau} \dot{p}_{\mathrm{C}} d t\right)=\frac{1}{\tau} \int_{0}^{\tau} \dot{p}(t) d t=\frac{1}{\tau}(p(\tau)-p(0)) .
$$

Pelas condições de continuidade que adotamos, $p(\tau)=p_{\mathrm{C}}(\tau)=p_{\mathrm{H}}(0)=p(0)$, assim, a expressão (3.1.14). pode ser reescrita como:

$$
\bar{J}_{\mathrm{H}}+\bar{J}_{\mathrm{C}}=0
$$


O mesmo resultado pode ser obtido integrando explicitamente as expressões em (3.1.9), tomando a solução geral encontrada (3.1.6,3.1.7). Assim:

$$
\bar{J}_{\mathrm{H}}=-\bar{J}_{\mathrm{C}}=\frac{1}{\tau} \frac{e^{-\left(\omega_{\mathrm{H}}+\bar{\omega}_{\mathrm{H}}\right) \tau_{\mathrm{H}}}\left[1-e^{-\left(\omega_{\mathrm{C}}+\bar{\omega}_{\mathrm{C}}\right)\left(\tau-\tau_{\mathrm{H}}\right)}\right]}{1-e^{-\left(\omega_{\mathrm{H}}+\bar{\omega}_{\mathrm{H}}\right) \tau_{\mathrm{H}}-\left(\omega_{\mathrm{C}}+\bar{\omega}_{\mathrm{C}}\right)\left(\tau-\tau_{\mathrm{H}}\right)}} \frac{\omega_{\mathrm{H}} \bar{\omega}_{\mathrm{C}}-\bar{\omega}_{\mathrm{H}} \omega_{\mathrm{C}}}{\left(\omega_{\mathrm{H}}+\bar{\omega}_{\mathrm{H}}\right)\left(\omega_{\mathrm{C}}+\bar{\omega}_{\mathrm{C}}\right)} .
$$

Assim, a média das correntes de probabilidades $\bar{J}$ é nula ao longo do ciclo. Podemos interpre$\operatorname{tar} \bar{J}_{i}$ para ambos os reservatórios como um fluxo de partículas, de forma que as expressões (3.1.15, 3.1.16) denotam a conservação delas: em média, o fluxo de entrada de partículas é igual ao fluxo de saída, uma vez que não é permitido acúmulo de partículas no quantum-dot. Quando $\bar{J}_{\mathrm{H}}>0\left(\overline{J_{\mathrm{C}}}<0\right)$, o fluxo de partícula vai do reservatório quente para o frio. Esse regime ocorre quando $\omega_{\mathrm{H}} / \bar{\omega}_{\mathrm{H}}>\omega_{\mathrm{C}} / \bar{\omega}_{\mathrm{C}}$. O oposto ocorre quando $\omega_{\mathrm{C}} / \bar{\omega}_{\mathrm{C}}>\omega_{\mathrm{H}} / \bar{\omega}_{\mathrm{H}}$. Podemos interpretar a fração $\omega_{i} / \bar{\omega}_{i}$ como a facilidade ou não do reservatório $i$ doar (ou receber) uma partícula para o (do) sistema. Como feito na seção (2.5), usaremos o fluxo de partícula para calcular explicitamente grandezas termodinâmicas como o fluxo de calor médio $\overline{\dot{Q}}$, fluxo de trabalho médio $\overline{\dot{W}}$ e a produção de entropia.

Conforme discutido anteriormente, desconsiderando a perturbação que causa a mudança de reservatórios, o sistema fermiônico interagindo com um único reservatório evolui para o equilíbrio termodinâmico após um certo tempo. Pelas expressões (3.1.6, 3.1.7), para um dado reservatório $i$, o limite para tempos longos da solução da equação mestra é dada por:

$$
p_{i}^{(\text {est })}=\frac{\omega_{i}}{\omega_{i}+\bar{\omega}_{i}}
$$

A conexão com as variáveis macroscópicas é considerada relacionando a razão entre as taxas $\omega_{i}$ e $\bar{\omega}_{i}$ com a probabilidade $p_{i}^{\text {eq }}$

$$
\frac{\omega_{i}}{\bar{\omega}_{i}}=e^{-\left(\epsilon-\mu_{i}\right) / T_{i}}
$$

que expressa o balanço detalhado local. Os valores $\mu_{i}$ e $\epsilon$ são respectivamente o potencial químico e a energia de ocupação do fermion. A partir dessa expressão, conseguimos escrever a temperatura $T_{i}$ de cada reservatório em função das taxas de transição $\omega_{i}$ e $\bar{\omega}_{i}$ :

$$
T_{i}=\frac{\mu_{i}-\epsilon}{\ln \left(\omega_{i} / \bar{\omega}_{i}\right)}
$$

Considerando a escala absoluta de temperatura, $T_{i}>0$, implicando que $\mu_{i}>\epsilon$ e $\omega_{i}>\bar{\omega}_{i}$ ou $\mu<\epsilon \mathrm{e} \omega_{i}<\bar{\omega}_{i}$ 
Podemos encontrar a produção de entropia média em cada ciclo, usando a definição de corrente $J_{\mathrm{H}}$. Tomando como base a expressão dada por (2.4.86), podemos escrever a produção de entropia para cada reservatório como:

$$
\Pi(t)=\left\{\begin{array}{l}
\Pi_{\mathrm{H}}(t)=k_{\mathrm{B}} J_{\mathrm{H}}(t) \ln \left(\frac{\omega_{\mathrm{H}}\left(1-p_{\mathrm{H}}(t)\right)}{\bar{\omega}_{\mathrm{H}} p_{\mathrm{H}}(t)}\right) ; t \in\left[0, \tau_{\mathrm{H}}\right], \\
\Pi_{\mathrm{C}}(t)=k_{\mathrm{B}} J_{\mathrm{C}}(t) \ln \left(\frac{\omega_{\mathrm{C}}\left(1-p_{\mathrm{C}}(t)\right)}{\bar{\omega}_{\mathrm{C}} p_{\mathrm{C}}(t)}\right) ; t \in\left[\tau_{\mathrm{H}}, \tau\right] .
\end{array}\right.
$$

Por meio dessa expressão, podemos encontrar a produção de entropia média ao longo de um ciclo:

$$
\bar{\Pi}=\frac{1}{\tau} \int_{0}^{\tau} \Pi(t) d t=\frac{1}{\tau}\left(\int_{0}^{\tau_{\mathrm{H}}} \Pi_{\mathrm{H}}(t) d t+\int_{\tau_{\mathrm{H}}}^{\tau} \Pi_{\mathrm{C}}(t) d t\right) .
$$

Considerando a equação mestra na forma (3.1.13) e as condições de contorno para a probabilidade, podemos reescrever a expressão acima como:

$$
\bar{\Pi}=\frac{k_{\mathrm{B}}}{\tau} \bar{J}_{\mathrm{H}} \ln \left(\frac{\omega_{\mathrm{H}} \bar{\omega}_{\mathrm{C}}}{\bar{\omega}_{\mathrm{H}} \omega_{\mathrm{C}}}\right) .
$$

\subsection{Descrição Termodinâmica}

Uma vez que a probabilidade de ocupação e o fluxo médio de partículas são conhecidos, podemos encontrar o fluxo de calor e trabalho realizado pela máquina, conforme apresentado na seção (2.5), de forma que escrevemos o fluxo de calor trocado entre o sistema e o reservatório depende do fluxo de partículas, da energia $\epsilon$ e do potencial químico de cada reservatório $\mu_{i}$, dado por:

$$
\overline{\dot{Q}}_{i}=\left(\epsilon-\mu_{i}\right) \bar{J}_{i}
$$

Pela expressão acima, não haverá fluxo de calor se o fluxo médio de partículas de cada reservatório for nulo. O fluxo de calor $\overline{\dot{Q}}_{\mathrm{H}}$ é doado para o sistema pelo reservatório quente. O fluxo $\overline{\dot{Q}}_{\mathrm{C}}$ é extraído do sistema pelo reservatóro frio. Uma vez que $\epsilon$ não varia no tempo no presente estudo, não haverá trabalho neste caso, de forma que será o trabalho químico resultante da troca de partículas entre os reservatórios a fonte para a extração de potência útil. Usamos a expressão (2.5.10) para calcular a sua média.

$$
\overline{\dot{W}}_{i}^{\text {chem }}=\mu_{i} \bar{J}_{i}
$$


assim como ocorre no fluxo de calor, o trabalho químico médio também é proporcional ao fluxo médio de partículas. Os fluxos para cada reservatório $i$ devem obedecer a primeira lei da termodinâmica. Adotaremos a seguinte condição de sinal:

$$
\overline{\dot{Q}}_{\mathrm{C}}+\overline{\dot{Q}}_{\mathrm{H}}+\overline{\dot{W}}_{\mathrm{C}}^{\text {chem }}+\overline{\dot{W}}_{\mathrm{H}}^{\text {chem }}=0 .
$$

A potência útil média é dada por:

$$
\bar{P}=-\left(\overline{\dot{W}}_{\mathrm{C}}^{\text {chem }}+\overline{\dot{W}}_{\mathrm{H}}^{\text {chem }}\right)
$$

A expressão acima mostra que a origem do trabalho útil extraído está relacionado com a troca de partículas entre o reservatório e o sistema. Essa ideia é similar ao conceito de particleexchange heat engine, conforme verificado experimentalmente por [23]. Dessa maneira, dada a expressão (3.1.19), podemos escrever o rendimento da máquina dada por:

$$
\eta=\frac{\bar{P}}{\dot{Q}_{\mathrm{H}}}=1-\frac{T_{\mathrm{C}}}{T_{\mathrm{H}}} \frac{\ln \left(\omega_{\mathrm{C}} / \bar{\omega}_{\mathrm{C}}\right)}{\ln \left(\omega_{\mathrm{H}} / \bar{\omega}_{\mathrm{H}}\right)} .
$$

Note que no caso particular onde $\omega_{\mathrm{C}} / \bar{\omega}_{\mathrm{C}}=\omega_{\mathrm{H}} / \bar{\omega}_{\mathrm{H}}$, a eficiência (3.2.5) se reduz a expressão de Carnot,

$$
\eta=1-\frac{T_{\mathrm{C}}}{T_{\mathrm{H}}}
$$

expressando o fato de máquinas térmicas reversíveis terem uma eficiência máxima. Também é importante notar que nesse caso em particular, a produção de entropia dada pela expressão (3.1.22) se anula, caracterizando o estado de equilíbrio. Com base na equação (3.1.18), podemos reescrever a equação (3.2.5) na forma:

$$
\eta=1-\frac{\mu_{\mathrm{C}}-\epsilon}{\mu_{\mathrm{H}}-\epsilon}=\frac{\mu_{\mathrm{C}}-\mu_{\mathrm{H}}}{\epsilon-\mu_{\mathrm{H}}} .
$$

O conjunto de valores $\left\{\mu_{i}, \epsilon, \bar{\omega}_{i}, \omega_{i}\right\}$ deve ser de tal modo que a segunda lei da termodinâmica seja garantida de acordo com o que enunciamos na seção (2.3). A eficiência deve ser sempre menor que um no regime de máquina. Tomando a expressão (3.2.7), temos que:

$$
0<\frac{\mu_{\mathrm{C}}-\mu_{\mathrm{H}}}{\epsilon-\mu_{\mathrm{H}}}<1
$$

Nesse regime, concluímos que $\omega_{\mathrm{C}} / \bar{\omega}_{\mathrm{C}}<\omega_{\mathrm{H}} / \omega_{\mathrm{H}}<1$, em consistência com a condição de que o fluxo de partículas vá do reservatório quente para o frio e o fluxo de calor do reservatório frio é negativo, $\overline{\dot{Q}}_{\mathrm{C}}<0$. 
Conforme mencionamos anteriormente, nosso objetivo visa em maximizar a potência média e a eficiência da máquina, considerarmos os protocolos estabelecidos pelas desigualdades acima. Existem várias formas de se estudar as maximizações. Alguns trabalhos anteriores consideram tempos de interação simétricos $\left(\tau_{\mathrm{H}}=\tau / 2\right)$ [21,22] e visam a maximização em termos das taxas de transição $\omega_{i}$ e $\bar{\omega}_{i}$. Neste capítulo exploraremos a influência de uma assimetria na interação do tempo $\left(\tau_{\mathrm{H}} \neq \tau / 2\right)$ e faremos a comparação com a maximização em relação aos $\omega_{i}^{\prime} s$. Consideraremos, nestes regimes, a fração $T_{\mathrm{C}} / T_{\mathrm{H}}$ fixa. Com a relação (3.1.18), conseguimos correlacionar as taxas de transição que permitem a maximização com as grandezas físicas $\epsilon$ e $\mu_{i}$ de cada reservatório.

\subsection{Protocolos de Maximização}

Inicialmente, começaremos a estudar o comportamento da potência em termos da duração das interações térmicas do sistema com os reservatórios. Neste caso, é interessante definir o conceito de tempo característico $t_{\text {char, dado por: }}$

$$
t_{\text {char }}=\max \left\{\frac{1}{\omega_{\mathrm{C}}+\bar{\omega}_{\mathrm{C}}}, \frac{1}{\omega_{\mathrm{H}}+\bar{\omega}_{\mathrm{H}}}\right\},
$$

onde $\left(\omega_{\mathrm{C}}+\bar{\omega}_{\mathrm{C}}\right)^{-1}$ e $\left(\omega_{\mathrm{H}}+\bar{\omega}_{\mathrm{H}}\right)^{-1}$ são tempos de relaxação do sistema quando interage, respectivamente, com os reservatórios $\mathrm{C}$ e $\mathrm{H}$. Para que o reservatório aja efetivamente no QD, é necessário que o sistema interaja durante um intervalo de tempo comparável ao seu tempo característico. Assim, o tempo característico pode nos dar uma noção de quanto tempo os reservatórios devem interagir com o QD para se obter uma máquina eficiente. Por exemplo, se $\left(\omega_{\mathrm{C}}+\bar{\omega}_{\mathrm{C}}\right)^{-1}<<\left(\omega_{\mathrm{H}}+\bar{\omega}_{\mathrm{H}}\right)^{-1}$, a interação entre o sistema com o reservatório quente deverá ser mais curta em comparação com reservatório frio, para a melhor extração de trabalho útil. Neste caso, portanto, espera-se um valor pequeno para a razão $\tau_{\mathrm{H}} / \tau$. Porém, espera-se também que, para $\tau_{\mathrm{H}} / \tau$ próximo de zero (e também para $\tau_{\mathrm{H}} / \tau$ próximo de $u m$ ), a potência média extraída seja aproximadamente nula, já que nesses regimes, o QD interage a maior parte do seu tempo com apenas um reservatório e quase nenhum trabalho útil pode ser extraído.

Note que de acordo com a equação (3.2.5) a eficiência não depende de $\tau, \tau_{\mathrm{H}}$ ou de $\tau_{\mathrm{C}}=\tau-\tau_{\mathrm{H}}$, de forma que a maximização dos parâmetros acima não afetarão a eficiência, 
mas afetarão a potência útil $\bar{P}$. Dessa forma, para maximizar a potência média encontrada na expressão (3.2.4), consideraremos o ponto crítico da potência em termos de $\tau_{\mathrm{H}}$ pela seguinte equação transcedental, que é obtida por derivação de $P$ por $\tau_{\mathrm{H}}$ :

$$
\frac{\left(\omega_{\mathrm{H}}+\bar{\omega}_{\mathrm{H}}\right)}{\left(\omega_{\mathrm{C}}+\bar{\omega}_{\mathrm{C}}\right)} \frac{e^{-\left(\omega_{\mathrm{H}}+\bar{\omega}_{\mathrm{H}}\right) \tau_{\mathrm{H}}}\left[1-e^{-\left(\omega_{\mathrm{C}}+\bar{\omega}_{\mathrm{C}}\right)\left(\tau-\tau_{\mathrm{H}}\right)}\right]^{2}}{e^{-\left(\omega_{\mathrm{C}}+\bar{\omega}_{\mathrm{C}}\right)\left(\tau-\tau_{\mathrm{H}}\right)}\left[1-e^{-\left(\omega_{\mathrm{H}}+\bar{\omega}_{\mathrm{H}}\right)\left(\tau_{\mathrm{H}}\right)}\right]^{2}}=1 .
$$

Existe um único valor de $\tau_{\mathrm{H}}$ que é solução da equação acima e maximiza potência média. Encontrar a solução da equação (3.3.2) para $\tau_{\mathrm{H}}$ é suficiente para garantir a máxima potência, isto é, não há necessidade do teste da segunda derivada, já que no intervalo onde ela é postiva (onde o sistema funciona como máquina, podendo ser extraído trabalho útil), a função $\bar{P}$ é sempre côncava, tendo como o único ponto crítico no intervalo o ponto de máximo local.

Fixando alguns valores para $T_{\mathrm{C}}, T_{\mathrm{H}}, \bar{\omega}_{i}$ e $\omega_{i}$, estudaremos os pontos que maximizam a

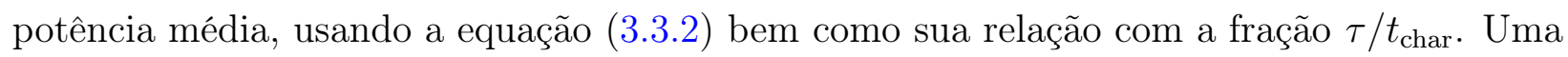
vez que a equação de maximização é transcedental, optamos por uma análise numérica de suas soluções, expressas nas figuras (3.1-3.4). Nessas figuras, expressamos os valores da potência média em termos de $\tau / t_{\text {char }}$ e $\tau_{\mathrm{H}} / \tau$, para vários protocolos (valores de $\omega_{i}$ e $\bar{\omega}_{i}$ ). Cada curva pontilhada preta representa uma variação de $10^{-4}$ no valor da potência. A curva pontilhada branca representa os pontos de $\tau / t$ e $\tau / t_{\text {char }}$ onde a potência é máxima em cada protocolo. Para cada gráfico, consideramos $\mu_{\mathrm{H}}=0.4, \mu_{\mathrm{C}}=0.6$ e a energia $\epsilon=1$. Nesse caso, considerando a expressão (3.2.7), a eficiência é fixa, resultando em $\eta=1 / 3$. 


\section{CAPÍTULO 3. MODELO COLISIONAL PARA UM SISTEMA DE QUANTUM DOT}

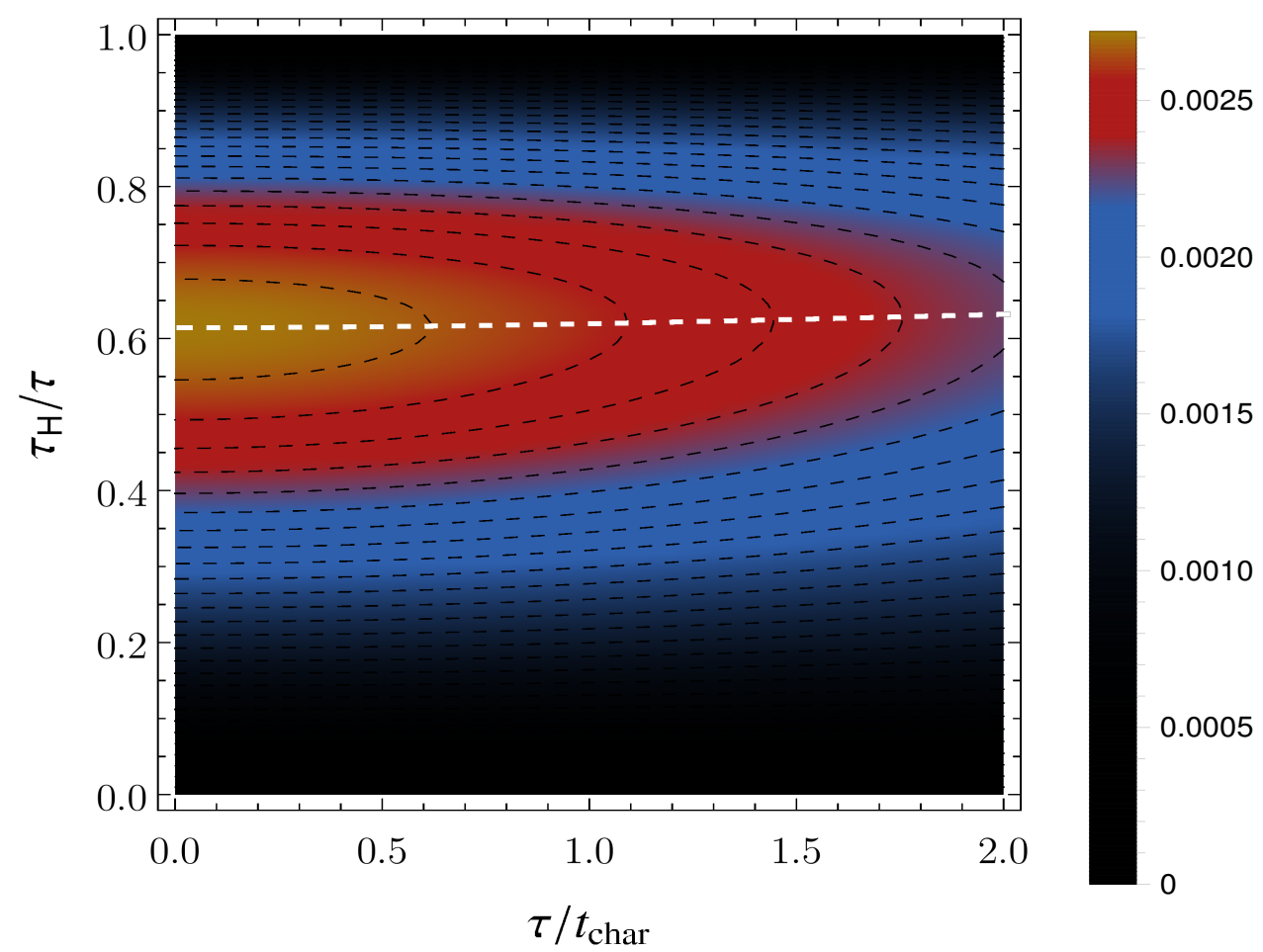

Figura 3.1: Valores da potência para diferentes valores das frações $\tau_{\mathrm{H}} / \tau$ e $\tau / t_{\text {char }}$ para $T_{\mathrm{H}}=$ $0.4, T_{\mathrm{C}}=0.1, \omega_{\mathrm{H}}=0.04, \bar{\omega}_{\mathrm{H}}=0.18, \omega_{\mathrm{C}}=0.01 \mathrm{e} \bar{\omega}_{\mathrm{C}}=0.55$. A barra lateral indica o valor da potência média $P$. 


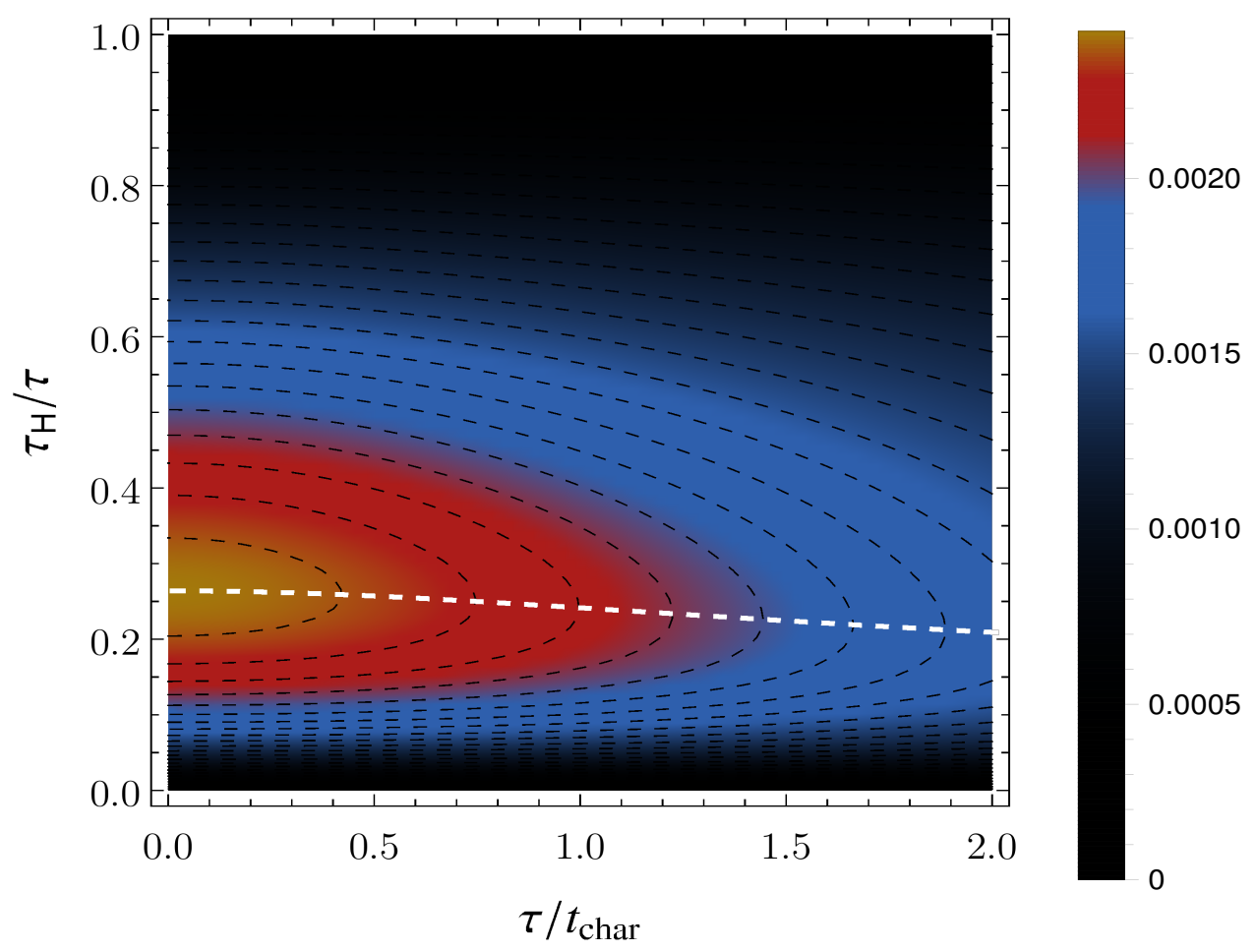

Figura 3.2: Valores da potência para diferentes valores das frações $\tau_{\mathrm{H}} / \tau$ e $\tau / t_{\text {char }}$ para $T_{\mathrm{H}}=$ $2.0, T_{\mathrm{C}}=0.5, \omega_{\mathrm{H}}=0.64, \bar{\omega}_{\mathrm{H}}=0.86, \omega_{\mathrm{C}}=0.06$ e $\bar{\omega}_{\mathrm{C}}=0.13$. A barra lateral indica o valor da potência média $P$. 


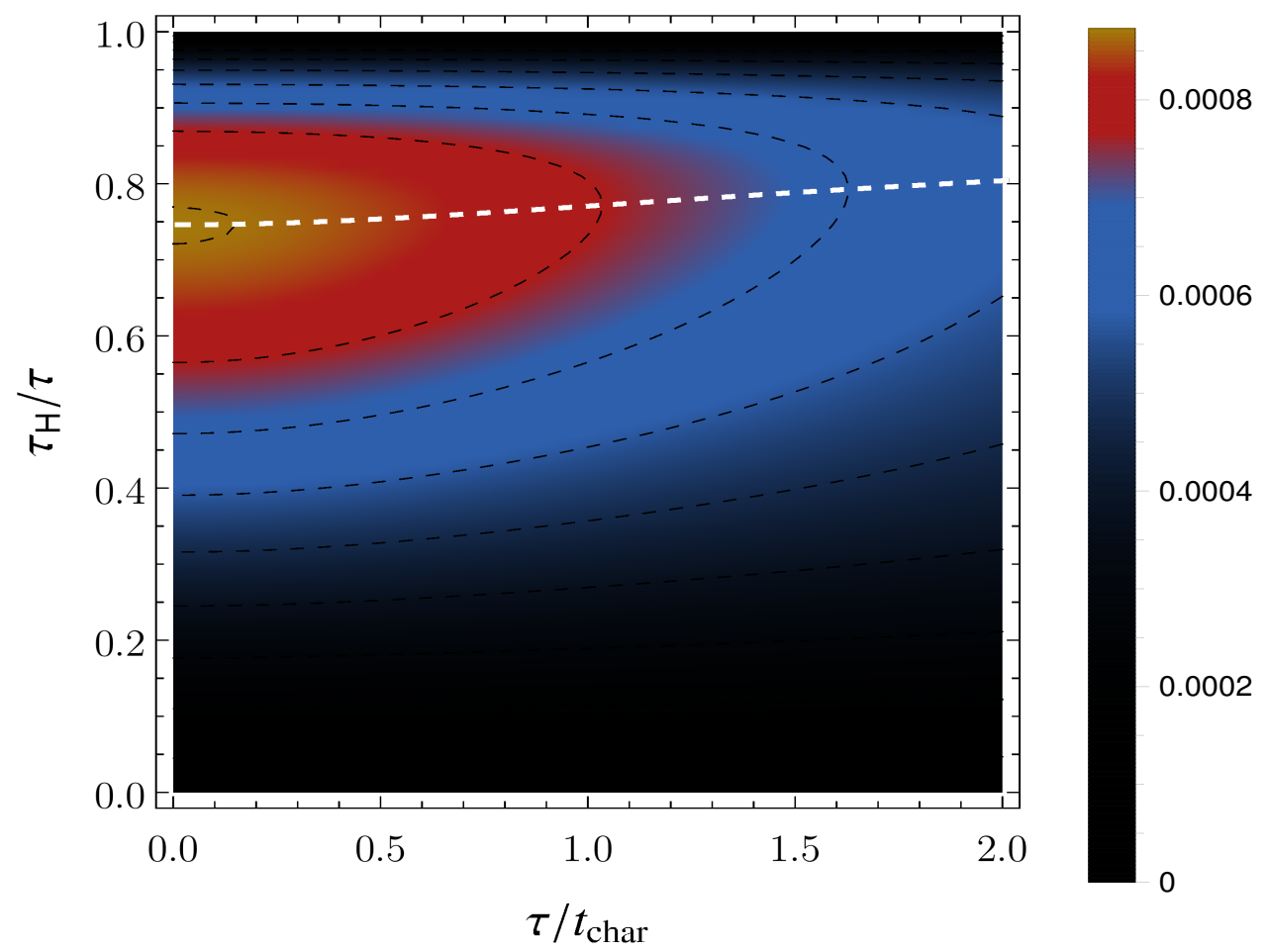

Figura 3.3: Valores da potência para diferentes valores das frações $\tau_{\mathrm{H}} / \tau$ e $\tau / t_{\text {char }}$ para $T_{\mathrm{H}}=$ $3.5, T_{\mathrm{C}}=1.2, \omega_{\mathrm{H}}=0.09, \bar{\omega}_{\mathrm{H}}=0.11, \omega_{\mathrm{C}}=0.71 \mathrm{e} \bar{\omega}_{\mathrm{C}}=0.99$. A barra lateral indica o valor da potência média $P$.

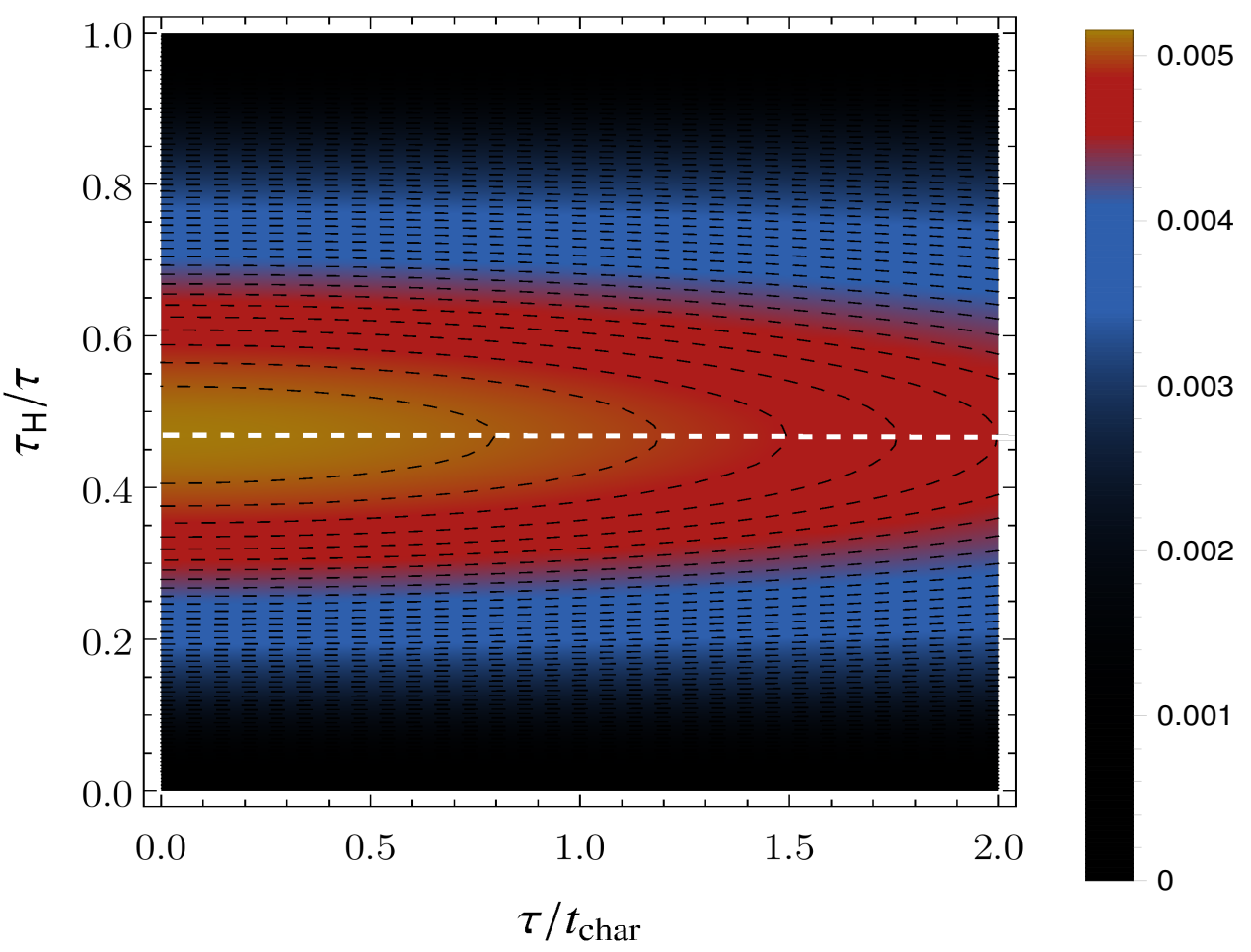

Figura 3.4: Valores da potência para diferenteg valores das frações $\tau_{\mathrm{H}} / \tau$ e $\tau / t_{\text {char }}$ para $T_{\mathrm{H}}=$ $0.4, T_{\mathrm{C}}=1.2, \omega_{\mathrm{H}}=0.13, \bar{\omega}_{\mathrm{H}}=0.58, \omega_{\mathrm{C}}=0.01 \mathrm{e} \bar{\omega}_{\mathrm{C}}=0.55$. A barra lateral indica o valor da potência média $P$. 
É interessante notar que, ao analisar as curvas pontilhadas brancas, os valores de $\tau_{\mathrm{H}} / \tau$ que maximizam a potência são numericamente pouco dependentes dos valores de $\tau / t_{\text {char }}$. Em contrapartida, muito variam em relação a outras características da máquina. Assim, podemos estabelecer três possíveis escalas de tempo para a nossa análise: a primeira envolve a fração $\tau_{\mathrm{H}} / \tau$, que representa a fração do tempo de interação entre o reservatório quente e o sistema de QD. A segunda envolve a análise do tempo característico de cada reservatório e o terceiro, envolve o tempo de interação necessário para garantir que as taxas de transição e a matriz de evolução $W_{i}$ sejam constantes durante a interação com cada reservatório. Esta última está relacionada com o tempo de troca entre a interação com os reservatórios quente e frio. No nosso modelo, consideramos que a terceira escala é muito pequena em comparação com as outras duas, de modo que ela pode ser negligenciada. Esta hipótese é assumida por trabalhos anteriores que também estudam máquinas de QD [21-24]. Assim, para e escolha de $\tau$, consideraremos o menor período possível, em concordância com [24], mas ainda assim maior que o tempo de troca entre os reservatórios, considerado aqui instantâneo. Também é interessante ressaltar que as curvas expressas pelas figuras anteriores corroboram a estimativa do tempo característico para maximização. Ao calcular a fração $\left(\omega_{\mathrm{H}}+\bar{\omega}_{\mathrm{H}}\right)^{-1} /\left[\left(\omega_{\mathrm{H}}+\bar{\omega}_{\mathrm{H}}\right)^{-1}+\right.$ $\left(\omega_{\mathrm{C}}+\bar{\omega}_{\mathrm{C}}\right)^{-1}$ ] para as figuras $(3.1,3.2,3.3,3.4)$, encontramos, respectivamente, os valores 0.7 , 0.1, 0.9 e 0.4, que são valores próximos aos da fração $\tau_{\mathrm{H}} / \tau$ nos mesmos regimes.

Como $\tau_{\mathrm{H}} / \tau$ que maximiza a potência pouco varia em relação aos correspondentes valores de $\tau / t_{\text {char }}$, consideraremos apenas a análise de $\tau_{\mathrm{H}}$ para a maximização da potência. Além disso, podemos notar pelas figuras (3.1-3.4) que a fração $\tau_{\mathrm{H}} / \tau$ que maximiza a potência podem ser diferentes de $1 / 2$, correspondendo assim a interações assimétricas entre os reservatórios. Em outras palavras, a maximização simétrica $\left(\tau_{\mathrm{H}} / \tau=1 / 2\right)$ pode ocorrer para um conjunto específicos de valores do protocolo.

Uma indagação que deve surgir nesto ponto é se a maximização considerando a interação $\tau_{\mathrm{H}}$ é mais interessante do que analisar outros protocolos fixando $\tau_{\mathrm{H}}=\tau / 2$. Para responder esse questionamento, faremos a comparação entre as potências em função da razão $T_{\mathrm{C}} / T_{\mathrm{H}}$, por meio da fração $P=\bar{P}\left(\tau_{\mathrm{H}}\right) / \bar{P}(\tau / 2)$. Usando os mesmos valores de potenciais químicos e energia adotados nas figuras (3.1-3.4), fixamos $T_{\mathrm{H}}, \bar{\omega}_{\mathrm{H}}$ e $\bar{\omega}_{\mathrm{C}}$. Tomando um ponto de $T_{\mathrm{C}} / T_{\mathrm{H}}$, os valores de $\omega_{\mathrm{C}}$ e $\omega_{\mathrm{H}}$ podem ser deduzidos pelo balanço detalhado local (3.1.18). Fixamos $\tau=1$, em concordância com a discussão anterior. 


\section{CAPÍTULO 3. MODELO COLISIONAL PARA UM SISTEMA DE QUANTUM DOT}

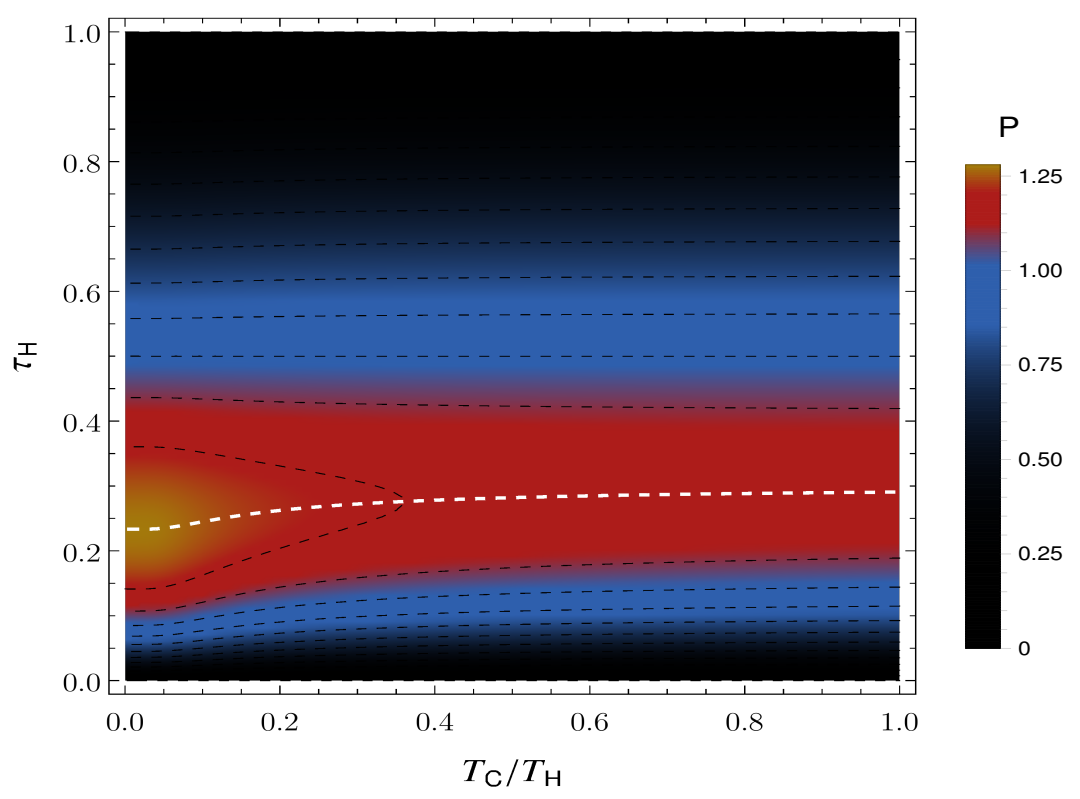

Figura 3.5: Valores da potência para diferentes valores de $\tau_{\mathrm{H}}$ versus $T_{\mathrm{C}} / T_{\mathrm{H}}$ para $T_{\mathrm{H}}=2.0$, $\bar{\omega}_{\mathrm{H}}=0.86, \bar{\omega}_{\mathrm{C}}=0.14$ e $\tau=1$. A barra lateral indica o valor da fração $P=\bar{P}\left(\tau_{\mathrm{H}}\right) / \bar{P}(\tau / 2)$.

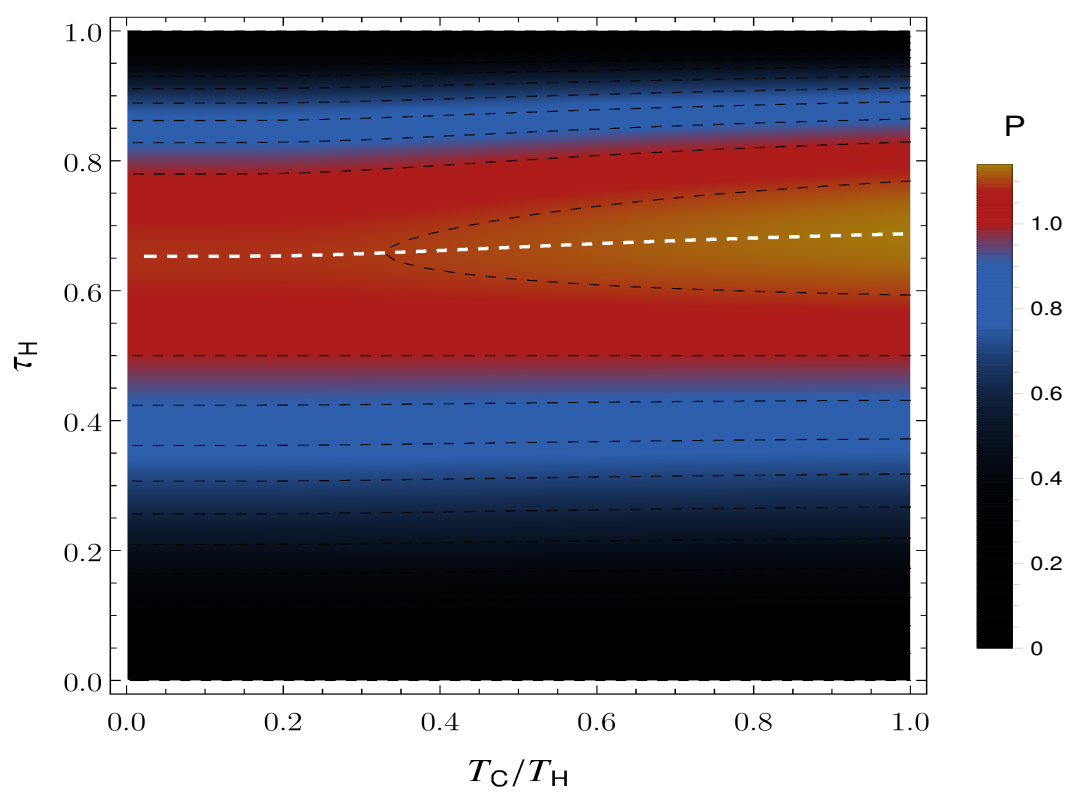

Figura 3.6: Valores da potência para diferentes valores de $\tau_{\mathrm{H}}$ versus $T_{\mathrm{C}} / T_{\mathrm{H}}$ para $T_{\mathrm{H}}=0.4$, $\bar{\omega}_{\mathrm{H}}=0.16$ e $\bar{\omega}_{\mathrm{C}}=0.69$. A barra lateral indica o valor da fração $P=\bar{P}\left(\tau_{\mathrm{H}}\right) / \bar{P}(\tau / 2)$. 


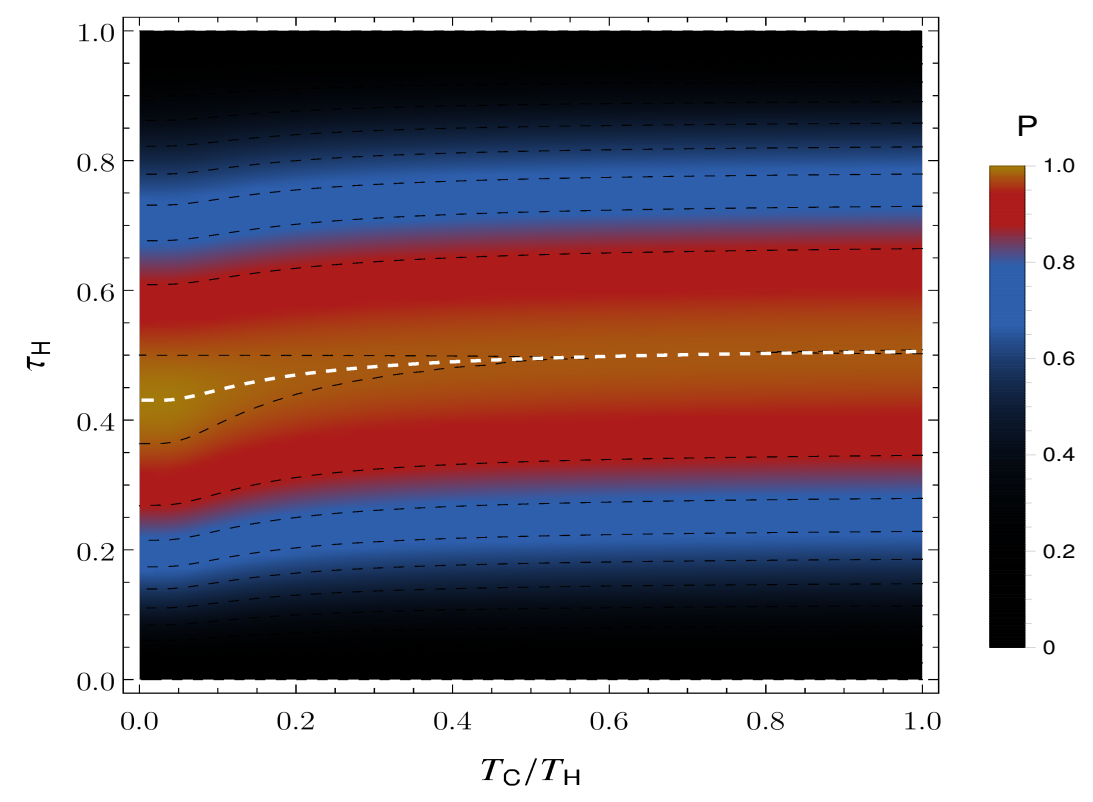

Figura 3.7: Valores da potência para diferentes valores de $\tau_{\mathrm{H}}$ versus $T_{\mathrm{C}} / T_{\mathrm{H}}$ para $T_{\mathrm{H}}=2.0$, $\bar{\omega}_{\mathrm{H}}=0.5$ e $\bar{\omega}_{\mathrm{C}}=0.5$. A barra lateral indica o valor da fração $P=\bar{P}\left(\tau_{\mathrm{H}}\right) / \bar{P}(\tau / 2)$.

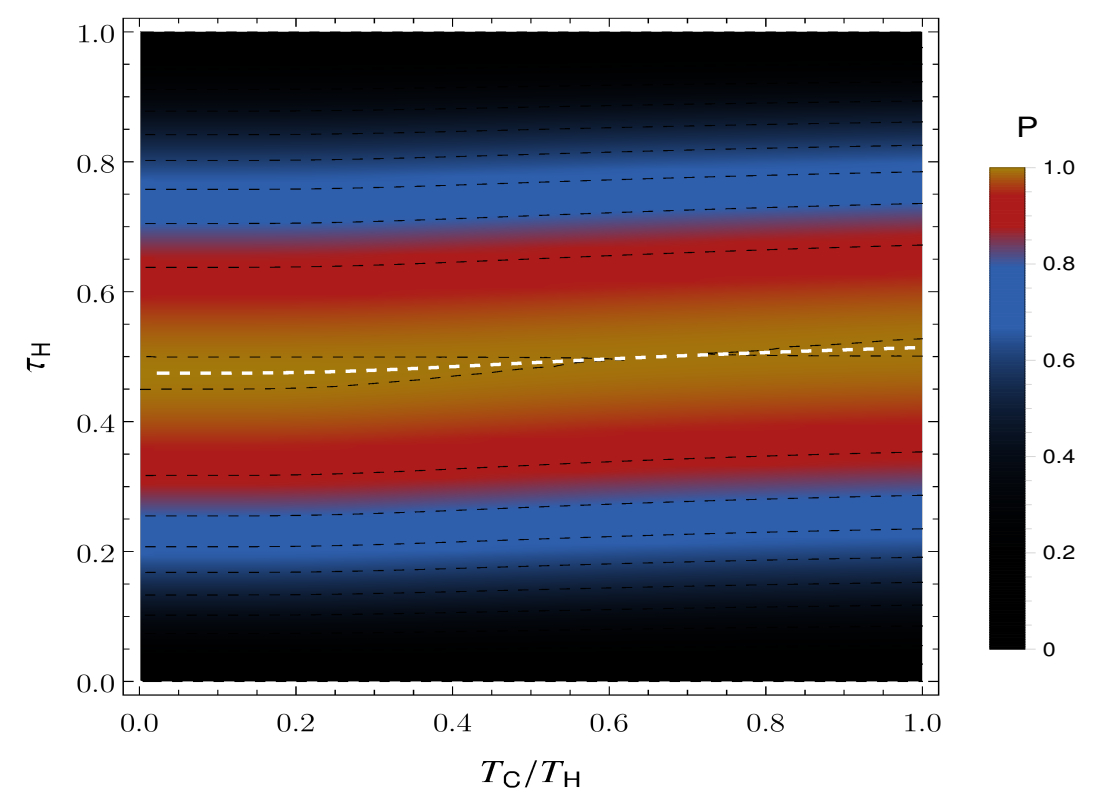

Figura 3.8: Valores da potência para diferentes valores de $\tau_{\mathrm{H}}$ versus $T_{\mathrm{C}} / T_{\mathrm{H}}$ para $T_{\mathrm{H}}=0.4$, $\bar{\omega}_{\mathrm{H}}=0.5$ e $\bar{\omega}_{\mathrm{C}}=0.5$. A barra lateral indica o valor da fração $P=\bar{P}\left(\tau_{\mathrm{H}}\right) / \bar{P}(\tau / 2)$.

A curva pontilhada branca representa a região de maior potência. Cada pontilhado preto signfica um incremento de 0.1 no valor da fração das potências. Podemos observar pelas 
figuras $(3.5,3.6)$ que a potência para tempos assimétricos é maior em até $25 \%$ em relação à potência para $\tau_{\mathrm{H}}=\tau / 2$. Porém, para os casos onde $\bar{\omega}_{\mathrm{C}}=\bar{\omega}_{\mathrm{H}}(3.7,3.8)$, observamos que a potência máxima ocorre em regiões próximas ao regime simétrico. Também podemos notar que para o caso onde $\bar{\omega}_{\mathrm{H}}>\bar{\omega}_{\mathrm{C}}$, a potência máxima é alcançada para regiões onde $T_{\mathrm{C}}>>T_{\mathrm{H}}$. O oposto ocorre para $\bar{\omega}_{\mathrm{H}}<\bar{\omega}_{\mathrm{C}}$. Como já discutimos, $\omega_{\mathrm{H}}>\omega_{\mathrm{C}}$ significa que mais partículas em média são doadas para o reservatório quente do que para o reservatório frio.

\subsection{Maximização Completa}

Discutimos na seção anterior a maximização da potência em termos dos protocolos da máquina (tempo de interação do sistema com a reservatório), fixando valores para as taxas de transição. Agora, adotaremos um método global: em relação a essas duas grandezas, qual delas oferece o melhor regime de otimização. A figura (3.9) nos dá uma base para essa maximização. Para construí-la, consideramos as frações $\tau_{\mathrm{H}} / \tau, \omega_{\mathrm{C}} / \bar{\omega}_{\mathrm{C}}$ e $\omega_{\mathrm{H}} / \bar{\omega}_{\mathrm{H}}$ em função de $T_{\mathrm{C}} / T_{\mathrm{H}}$. Para diferentes valores de $\bar{\omega}_{\mathrm{H}}$ e $\bar{\omega}_{\mathrm{C}}$ que variam entre 0.1 e 2.0 com intervalo de 0.05 de um valor a outro, tomamos um par de taxas de transição $\left(\bar{\omega}_{\mathrm{H}}, \bar{\omega}_{\mathrm{C}}\right)$, isto é, tomamos valores de $\bar{\omega}_{\mathrm{C}}$ e $\bar{\omega}_{\mathrm{H}}$ entre 0.1 e 2 , variando 0.05 a cada valor. A partir daí, encontramos a maior potência para esses valores escolhidos, para dados $T_{\mathrm{C}} / T_{\mathrm{H}}$ e $\tau$ previamente fixados. Os valores para $\tau_{\mathrm{H}} / \tau, \omega_{\mathrm{H}} / \bar{\omega}_{\mathrm{H}}$ e $\omega_{\mathrm{C}} / \bar{\omega}_{\mathrm{C}}$ onde são registrados a maior potência são expressas nas curvas a seguir. Seus valores máximos, médios e mínimos em função de $T_{\mathrm{C}} / T_{\mathrm{H}}$ são dados pelas três curvas expressas na figura (3.9). Fixamos $\tau=1$ e $T_{\mathrm{H}}=1$. Valores intermediários dessas frações que também maximizam a potência estão na áreas hachuradas delimitadas pelas curvas máximas e mínimas. 


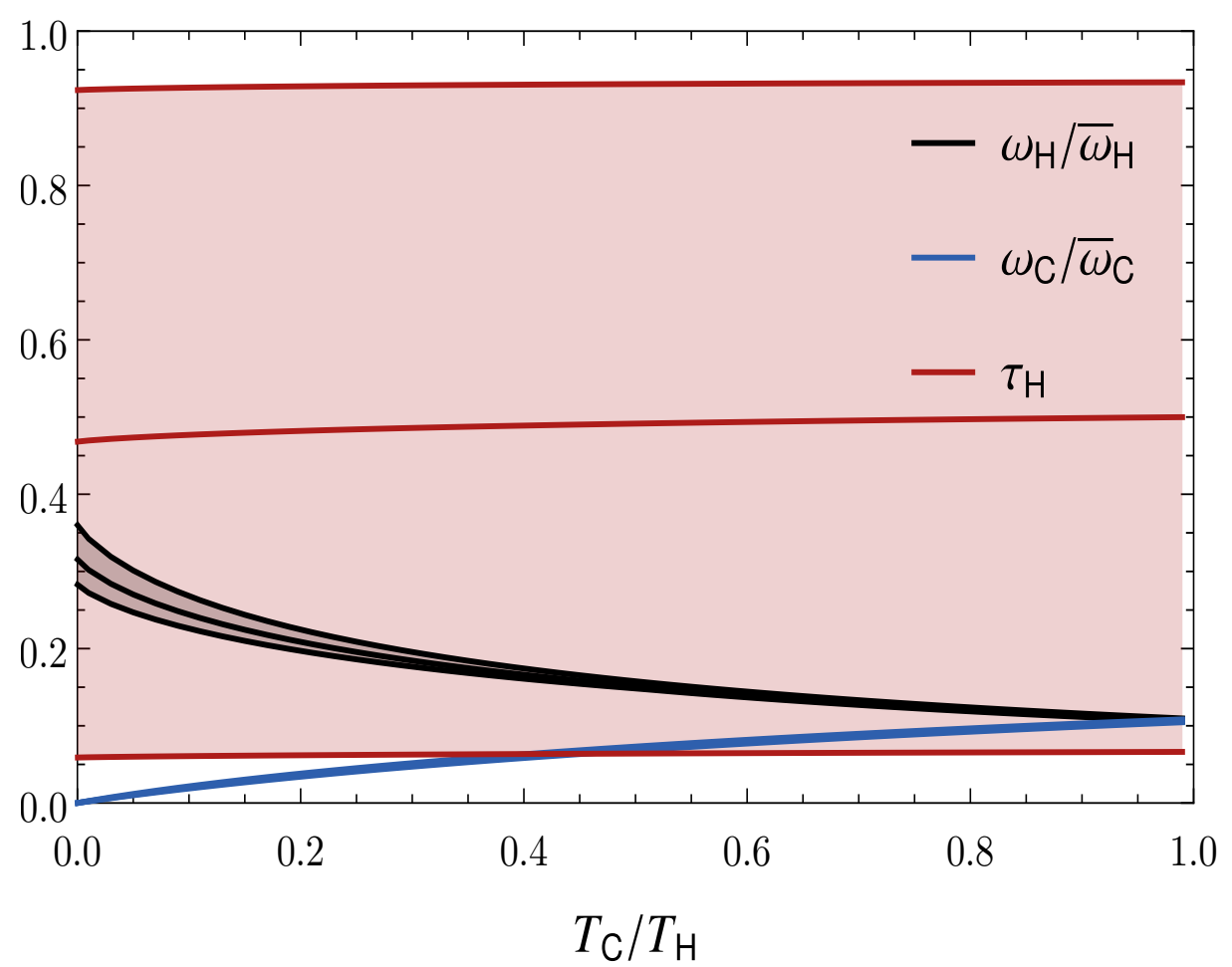

Figura 3.9: Valores de $\tau_{\mathrm{H}} / \tau$ (curva vermelha), $\omega_{\mathrm{H}} / \bar{\omega}_{\mathrm{H}}$ (curva preta) e $\omega_{\mathrm{C}} / \bar{\omega}_{\mathrm{C}}$ (curva azul) após a maximização da potência para temperaturas fixas e $\tau=1$. Os valores máximos, médios e mínimos para cada quantidade são dadas pelas três curvas, da maior para a menor. Valores intermediários que também maximizam a potência estão entre as áreas delimitadas pelas curvas.

O resultado expresso na Figura (3.9) revela que $\tau_{\mathrm{H}}$ é muito mais sensível em relação a troca de valores das taxas de transição $\bar{\omega}_{\mathrm{C}}$ e $\bar{\omega}_{\mathrm{H}}$, de forma que os valores de $\omega_{\mathrm{C}} / \bar{\omega}_{\mathrm{C}}$ e $\omega_{\mathrm{H}} / \bar{\omega}_{\mathrm{H}}$ pouco variam em relação a troca desses parâmetros. No caso da curva correspondente à fração $\omega_{\mathrm{C}} / \bar{\omega}_{\mathrm{C}}$ (curva azul), as curvas de máximo, mínimo e média coincidem na escala do gráfico. Isso revela a importância de se considerar o tempo de interação ao se estudar a maximização de potência da máquina, de forma que sua escolha é mais importante na maximização da potência em termos de outros parâmetros. Essa conclusão é reforçada pelos resultados expressos na Figura (3.10). Usando o mesmo grid adotado para $\bar{\omega}_{\mathrm{H}}$ e $\bar{\omega}_{\mathrm{C}}$, expressamos $\bar{P}_{\max }$, inicialmente maximizando em termos de apenas $\tau_{\mathrm{H}}$ (curvas contínuas) e depois uma maximizando globalmente, considerando $\tau_{\mathrm{H}}, \omega_{\mathrm{C}}$ e $\omega_{\mathrm{H}}$ (curvas tracejadas). 


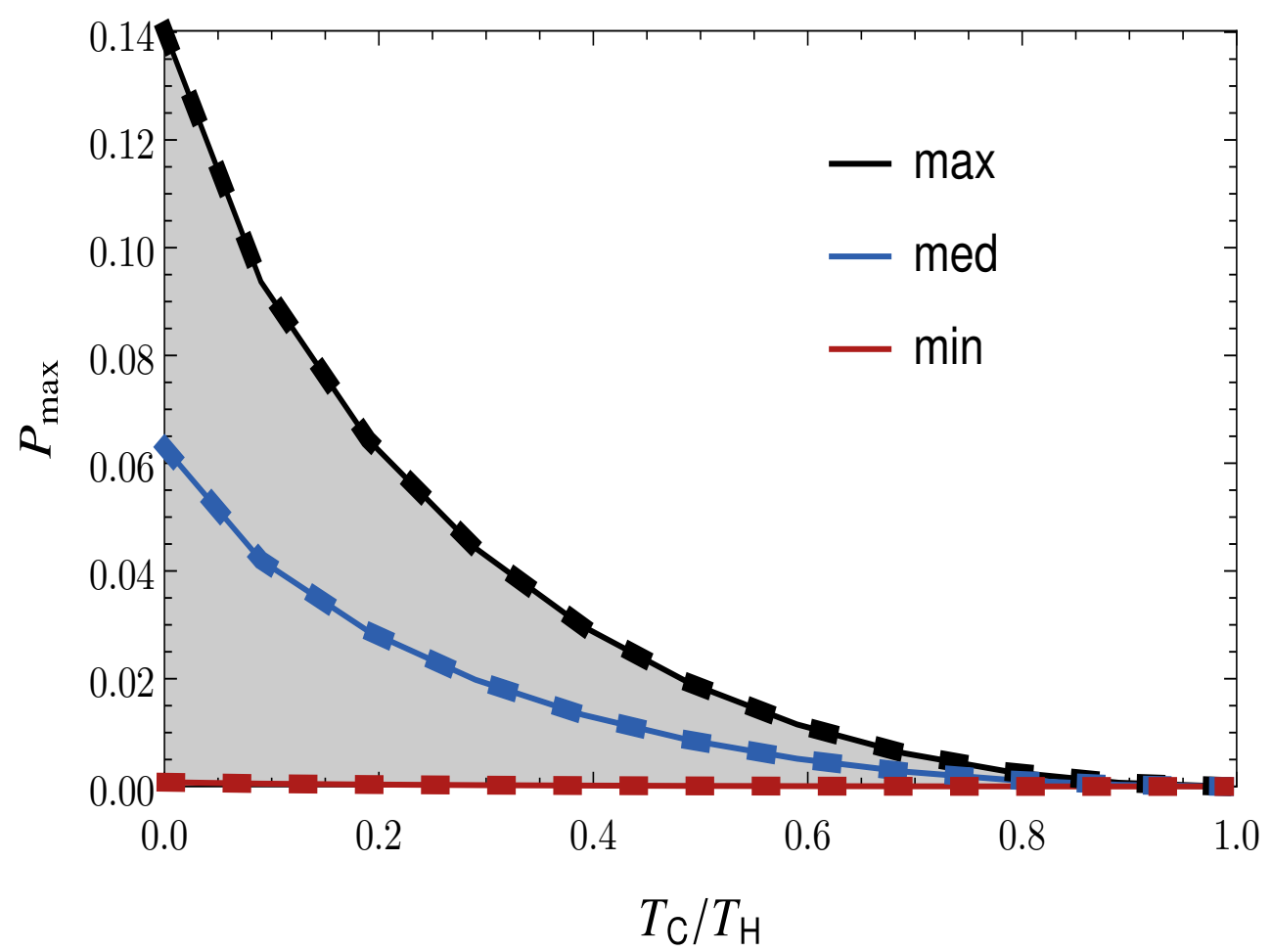

Figura 3.10: $\bar{P}_{\max }$ em relação a $T_{\mathrm{C}} / T_{\mathrm{H}}$ para $\tau=1$ com o mesmo grid da Fig. (3.9). As curvas pretas, azuis e vermelhas representam, respectivamente, o valor máximo, médio e mínimo de $P_{\max }$. Linhas contínuas representam a maximização apenas em termos de $\tau_{\mathrm{H}}$ e linhas pontilhadas representam maximização tem termos de $\tau_{\mathrm{H}}, \omega_{\mathrm{C}}$ e $\omega_{\mathrm{H}}$

Notamos que as diferenças entre as maximizações são muito pequenas. Mais especificamente, nota-se pelo gráfico expresso na Figura (3.10) que a potência máxima obtida apenas otimizando em relação a $\tau_{\mathrm{H}}$ isoladamente é aproximadamente igual àquela obtida na maximização global (com relação a $\tau_{\mathrm{H}}, \omega_{\mathrm{C}}$ e $\omega_{\mathrm{H}}$ ). Na escala do gráfico, elas são coincidentes em seus valores máximos, médios e mínimos. A diferença entre eles se dá pelo aumento dos valores de $\bar{\omega}_{\mathrm{C}}$ e $\bar{\omega}_{\mathrm{H}}$, que também influenciam na maximização.

Compararemos agora $\eta_{\mathrm{MP}}$, isto é a eficiência da máquina para máxima potência, com $\eta_{\mathrm{CA}}$ e $\eta_{\mathrm{C}}$, sendo respectivamente as eficiência de Curzon-Alhborn (CA) e Carnot (C). Fazemos a comparação $\eta_{\mathrm{MP}} / \eta_{\mathrm{CA}}$ em relação a $T_{\mathrm{C}} / T_{\mathrm{H}}$ na Figura (3.11). Repetimos o mesmo grid para $\bar{\omega}_{\mathrm{C}}$ e $\bar{\omega}_{\mathrm{H}}$. Com exceção de alguns valores de $\bar{\omega}_{\mathrm{C}}$ e $\bar{\omega}_{\mathrm{H}}$ em um pequeno intervalo da fração $T_{\mathrm{C}} / T_{\mathrm{H}}$ entre 0.4 e 1.0, a maximização fornece uma eficiência um pouco maior que 
a da CA. Isso nos confere uma segunda vantagem da maximização com relação ao período assimétrico. Como já foi discutido neste trabalho, embora a eficiência de CA não tenha o mesmo valor de universidade que a eficiência de Carnot e eficiências que ultrapassam seu valor já sejam conhecidas na literatura [17], ela é importante pelo fato de depender apenas das temperaturas dos reservatórios e não dos detalhes da máquina, além ser uma boa aproximação para a eficiência máxima para a maioria dos casos.

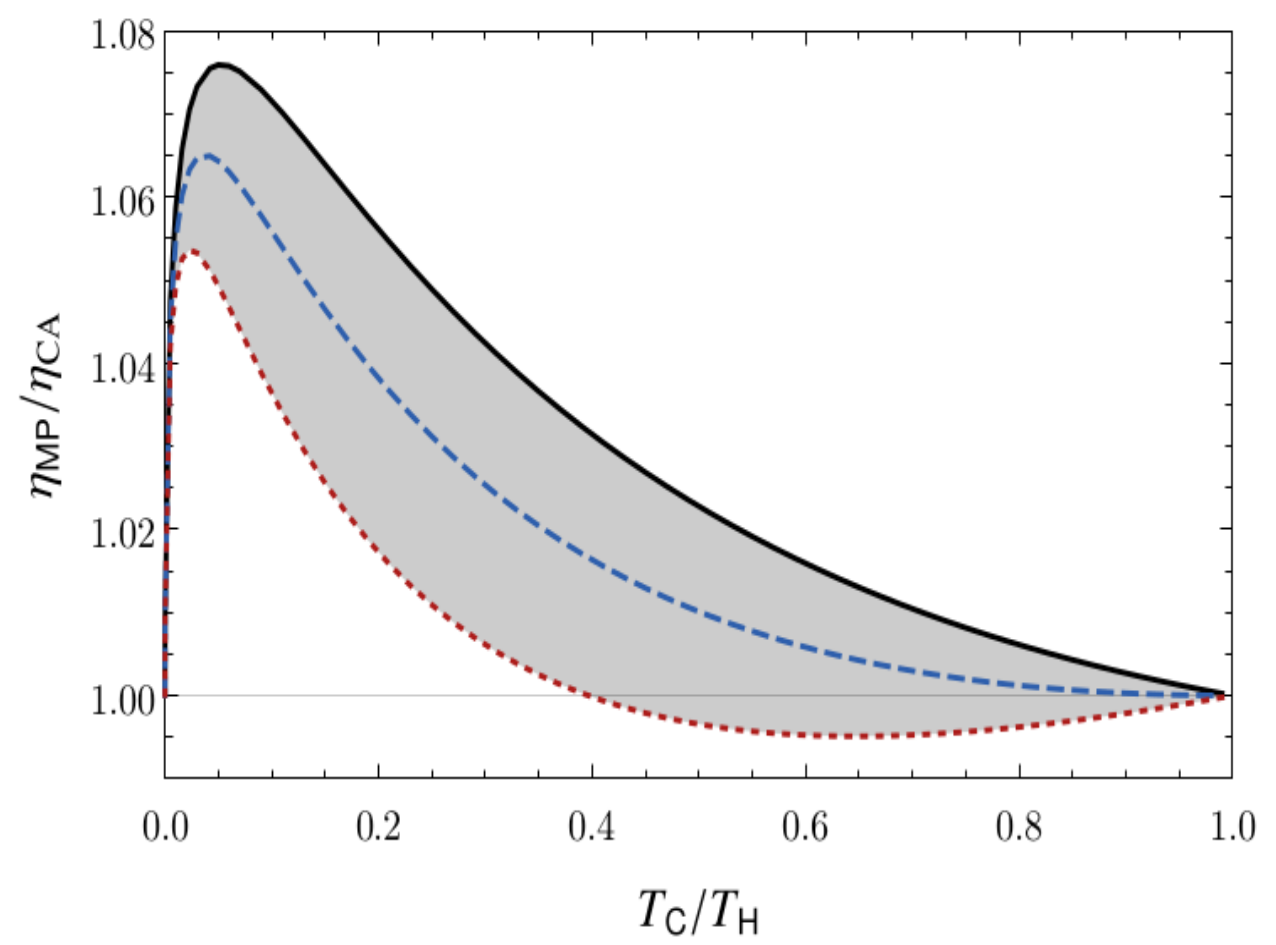

Figura 3.11: Razão entre $\eta_{\mathrm{MP}}$ e $\eta_{\mathrm{CA}}$ em relação $T_{\mathrm{C}} / T_{\mathrm{H}}$ para $\epsilon=1$ e $\tau=1$. A curva perta (linha contínua) representa a eficiência máxima local. Os valores médios e mínimos são representados por, respectivamente, pelos tracejados azuis e pontos vermelhos. Todas as curvas (e valores intermediários) são obtidos para o mesmo grid adotado na Fig.(3.9).

Finalmente, $\eta_{\mathrm{MP}}$ é sempre menor que ela, como deve ser. A Figura (3.12) expressa essa comparação. Note que $\eta_{\mathrm{MP}}$ é assintoticamente igual à $\eta_{\mathrm{C}}$ nos limites $T_{\mathrm{C}}<<T_{\mathrm{H}}$ e no limite $T_{\mathrm{C}} \rightarrow T_{\mathrm{H}}, \eta_{\mathrm{MP}} \rightarrow \frac{1}{2} \eta_{\mathrm{C}}$, como esperado, considerando as aproximações feitas em (2.3.20, 2.3.21). 


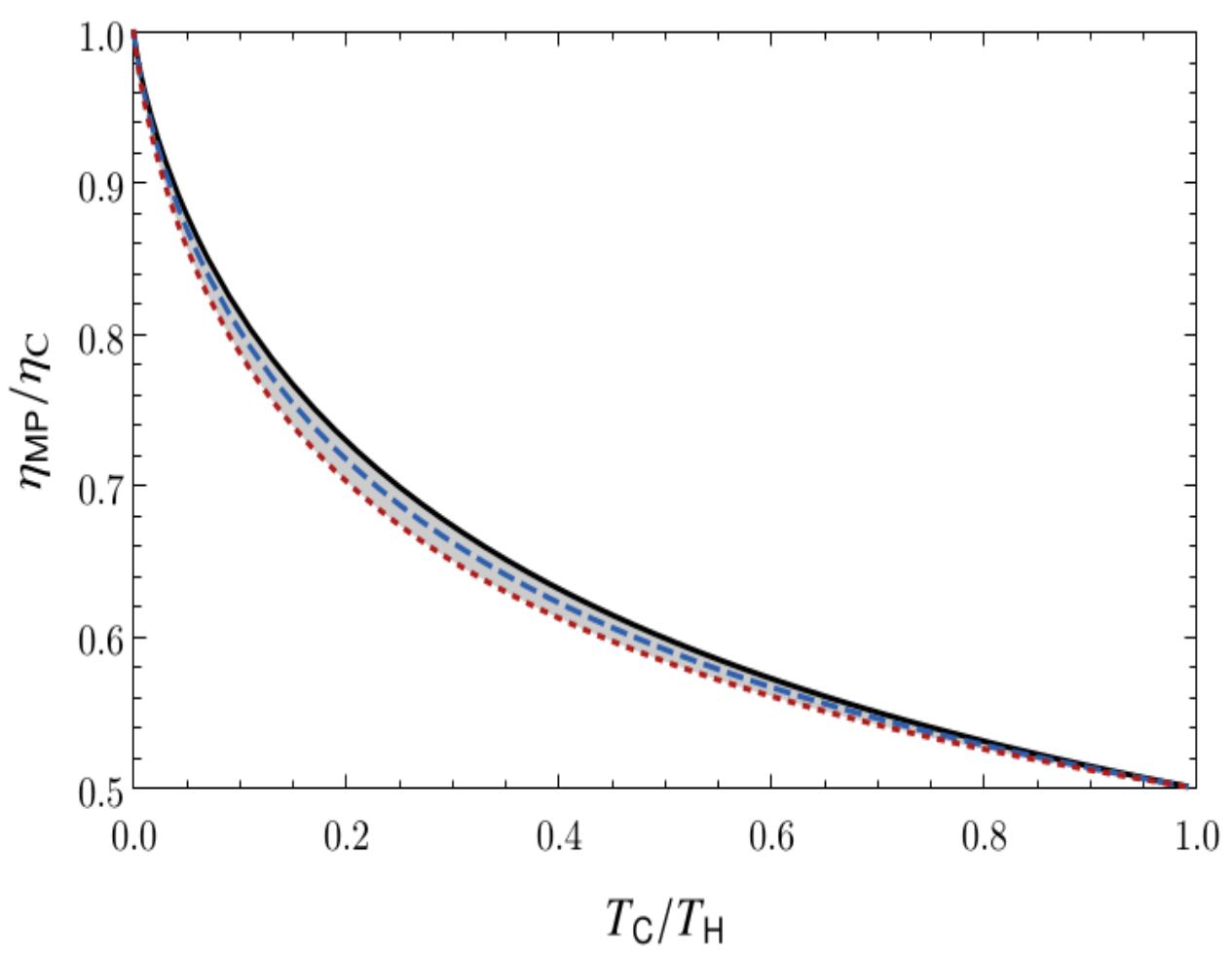

Figura 3.12: Razão entre o $\eta_{\mathrm{MP}}$ e $\eta_{\mathrm{C}}$ para diferentes valores de $T_{\mathrm{C}} / T_{\mathrm{H}}$, considerando as mesmas condições que a Fig (3.11).

Em suma, apresentamos um estudo sobre o papel de intervalos de tempo assimétricos num modelo colisional para o" quantum-dot". Tal sistema tem sido intensamente estudado na literatura para fins de máquinas térmicas, refrigeradores, dentre outros. Mostramos que a maximização do tempo de contato entre o sistema e o reservatório constitui uma estratégia bastante apropriada para maximizar a potência. Como um ganho adicional, a eficiência em alguns casos é superior a $\eta_{\mathrm{CA}}$. 


\section{Capítulo 4}

\section{Modelo Colisional para uma Partícula Browniana}

Consideraremos uma extensão da ideia de interação colisional aplicada agora a uma partícula Browniana que interage sequencialmente com reservatórios térmicos. Analogamente ao que foi tratado no capítulo anterior, o processo ocorre em um ciclo, onde a interação com o primeiro reservatório dura um determinado intervalo de tempo e depois é abruptamente forçado a entrar em contato com o segundo reservatório,. Após um tempo, o sistema volta para o primeiro, compondo assim um ciclo termodinâmico. Neste caso, não há troca de partículas entre o sistema e o reservatório e a extração de trabalho útil se dá por meio de uma força externa ao sistema. Estando apenas em contato com um único reservatório, o sistema evoluirá para o equilíbrio no regime de tempos longos, tendo sua produção de entropia anulada. A perturbação que força o sistema a mudar de reservatório tira o sistema do equilíbrio termodinâmico constantemente de forma que a solução da equação de FokkerPlanck apresenta uma forma estacionária (para tempos longos) de não equilíbrio (NonEquilibrium Steady State, NESS). Para esse regime, a produção de entropia média é não negativa. Em outras palavras, o sistema produz entropia continuamente. Nosso objetivo aqui consiste em analisar no caso mais simples (partículas livres com amortecimento supercrítico) o papel do "drives"(força externa) que maximiza a potência e a eficiência. Inicialmente, reproduzimos alguns resultados contidos na referência [25] para tempos simétricos e para alguns exemplos de forças externas (constates e lineares). Em seguida, iremos ao nosso 
objetivo de analisar o papel da força externa para diversas dependências temporais.

\subsection{Modelo e Solução Exata}

Consideramos dois reservatórios térmicos de temperatura $T_{1}$ e $T_{2}$, sendo respectivamente as temperaturas dos reservatórios quente e frio $\left(T_{1}>T_{2}\right)$. A interação térmica entre os dois ocorre em ciclos com período $\tau$. A interação com o primeiro ocorre durante um intervalo de tempo $\tau_{1}=\tau / 2$ e após a mudança instatânea de reservatório, a interação com o segundo ocorre durante o intervalo de tempo $\tau_{2}=\tau-\tau_{1}=\tau / 2$. Consideramos que em cada reservatório $i(i \in\{1,2\})$, há uma força externa ao sistema $F_{i}(t)=m f_{i}(t)$ interagindo com ele. De acordo com o que discutimos na seção (2.6.1), escrevemos a equação de Langevin para cada reservatório $i$ como:

$$
\begin{gathered}
\dot{v}_{i}(t)=-\gamma_{i} v_{i}(t)+f_{i}(t)+\zeta_{i}(t), \\
\frac{d x_{i}(t)}{d t}=v_{i}(t) .
\end{gathered}
$$

Os valores médios de $v_{i}(t)$ e $x_{i}(t)$ podem ser encontrados de maneira análoga ao discutido na seção (2.6.1). Como foi discutido no capítulo anterior, a continuidade da distribuição de probabilidade do sistema deve ser garantida, de forma que a distribuição de probabilidade $P_{i}(v, t)$ para cada reservatório térmico $i$ seja solução da equação de Fokker-Planck:

$$
\frac{\partial P_{i}}{\partial t}=-\frac{\partial J_{i}}{\partial v}-f_{i}(t) \frac{\partial P_{i}}{\partial v}
$$

com:

$$
J_{i}(t)=-\gamma v_{i}(t)-\frac{\Gamma_{i}}{2} \frac{\partial P}{\partial v}
$$

Embora o sistema evolua para um NESS, a distribuição de probabilidade é ainda da forma Gaussiana:

$$
P_{i}(v, t)=\frac{\exp \left(-\left(v_{i}(t)-\left\langle v_{i}(t)\right\rangle\right)^{2} / 2 b_{i}(t)\right)}{\sqrt{2 \pi b_{i}(t)}},
$$

agora com diferentes valores de $\left\langle v_{i}(t)\right\rangle$ e $b_{i}(t)$. Assim, as condições de contorno que expressam a continuidade da distribuição são aplicadas para essas grandezas. Estudaremos a evolução, 


\section{CAPÍTULO 4. MODELO COLISIONAL PARA UMA PARTÍCULA BROWNIANA}

para cada reservatório, da média da velocidade da variância pelas equações (2.6.49) e (2.6.50):

$$
\begin{aligned}
\frac{d\left\langle v_{i}(t)\right\rangle}{d t} & =-\gamma\left\langle v_{i}(t)\right\rangle+f_{i}(t), \\
\frac{d b_{i}(t)}{d t} & =-2 \gamma b_{i}(t)+\Gamma_{i} .
\end{aligned}
$$

As condições de continuidade de $P(v, t)$ exigem que a variância e a velocidade sejam iguais para os dois reservatórios no instante da mudança: $b_{1}(\tau / 2)=b_{2}(\tau / 2)$ e $\left\langle v_{1}(\tau / 2)\right\rangle=\left\langle v_{2}(\tau / 2)\right\rangle$. Além disso, a média da velocidade e a variância finais devem ser iguais aos valores iniciais, devido ao comportamento cíclico. Assim: $b_{2}(\tau)=b_{1}(0)$ e $\left\langle v_{2}(\tau)\right\rangle=\left\langle v_{1}(0)\right\rangle$. Dessa forma, para um ciclo e considerando as condições impostas acima, temos:

$$
\begin{aligned}
\left\langle v_{1}(t)\right\rangle & =e^{-t \gamma}\left[\int_{0}^{t} e^{-\gamma t^{\prime}} f_{1}\left(t^{\prime}\right) d t^{\prime}+\frac{1}{e^{\gamma \tau}-1}\left(\int_{0}^{\tau / 2} e^{\gamma t^{\prime}} f_{1}\left(t^{\prime}\right) d t^{\prime}+e^{\gamma \tau / 2} \int_{\tau / 2}^{\tau} e^{\gamma\left(t^{\prime}-\tau / 2\right)} f_{2}\left(t^{\prime}\right) d t^{\prime}\right)\right] \\
& t \in[0, \tau / 2] \\
\left\langle v_{2}(t)\right\rangle & =e^{-\gamma(t-\tau / 2)}\left\{\int_{\tau / 2}^{t} e^{\gamma(x-\tau / 2)} f_{2}\left(t^{\prime}\right) d t^{\prime}+e^{-\gamma \tau / 2}\left[\int_{0}^{\tau / 2} e^{\gamma t^{\prime}} f_{1}\left(t^{\prime}\right) d t^{\prime}+\right.\right. \\
& \left.\left.+\frac{1}{e^{\gamma \tau}-1}\left(\int_{0}^{\tau / 2} e^{\gamma t^{\prime}} f_{1}\left(t^{\prime}\right) d t^{\prime}+e^{\gamma \tau / 2} \int_{\tau / 2}^{\tau} e^{\gamma\left(t^{\prime}-\tau / 2\right)} f_{2}\left(t^{\prime}\right) d t^{\prime}\right)\right]\right\} t \in[\tau / 2, \tau]
\end{aligned}
$$

Para as médias e

$$
\begin{aligned}
& b_{1}(t)=\frac{\Gamma_{1}}{2 \gamma}-\frac{\left(\Gamma_{1}-\Gamma_{2}\right)}{2 \gamma\left(1+e^{\gamma \tau}\right)} e^{-2 \gamma t+\gamma \tau} ; t \in[0, \tau / 2], \\
& b_{2}(t)=\frac{\Gamma_{2}}{2 \gamma}+\frac{\left(\Gamma_{1}-\Gamma_{2}\right)}{2 \gamma\left(1+e^{\gamma \tau}\right)} e^{-2 \gamma(t-\tau / 2)+\gamma \tau} ; t \in[\tau / 2, \tau],
\end{aligned}
$$

para as variâncias, respectivamente. É interessante notar que a expressão da variância é independente da força externa aplicada.

A força $f_{i}(t)$ pode ser dadas por funções arbitrárias com o tempo $t$. Neste capítulo, focaremos em forças externas na forma:

$$
f(t)=\left\{\begin{array}{l}
X_{1} g_{1}(t) ; t \in[0, \tau / 2[, \\
X_{2} g_{2}(t) ; t \in[\tau / 2, \tau[
\end{array}\right.
$$

onde estudaremos a influência das funções $g_{i}(t)$ nas principais grandezas termodinâmicas, como a eficiência e a potência média, para alguns valores de drives $X_{1}$ e $X_{2}$. 


\subsection{Descrição Termodinâmica}

Uma vez obtidos os valores de $\left\langle v_{i}(t)\right\rangle$ e $\left\langle v_{i}^{2}(t)\right\rangle$, podemos obter as principais propriedades termodinâmicas, bem como a eficiência $\eta$. Iniciaremos por definir a eficiência $\eta$ para uma máquina browniana colisional. Tomando as definições de trabalho e calor dadas por (2.6.42, 2.6.43), escrevemos para cada reservatório:

$$
\begin{gathered}
\dot{W}(t)=\left\{\begin{array}{l}
\dot{W}_{1}(t)=-m f_{1}(t)\left\langle v_{1}(t)\right\rangle ; t \in[0, \tau / 2[, \\
\dot{W}_{2}(t)=-m f_{2}(t)\left\langle v_{2}(t)\right\rangle ; t \in[\tau / 2, \tau[,
\end{array}\right. \\
\dot{Q}(t)=\left\{\begin{array}{l}
\dot{Q}_{1}(t)=m \gamma\left(\left\langle v_{1}^{2}(t)\right\rangle-\frac{\Gamma_{1}}{2 \gamma}\right) ; t \in[0, \tau / 2[, \\
\dot{Q}_{2}(t)=m \gamma\left(\left\langle v_{2}^{2}(t)\right\rangle-\frac{\Gamma_{2}}{2 \gamma}\right) ; t \in[\tau / 2, \tau[,
\end{array}\right.
\end{gathered}
$$

respectivamente. Conforme fizemos no capítulo anterior, seus valores médios ao longo de um ciclo são dados por:

$$
\begin{aligned}
& \overline{\dot{W}}=\frac{1}{\tau}\left(\int_{0}^{\tau} \dot{W}(t) d t\right)=\frac{1}{\tau}\left(\int_{0}^{\tau / 2} \dot{W}_{1}(t) d t+\int_{\tau / 2}^{\tau} \dot{W}_{2}(t) d t\right)=\overline{\dot{W}}_{1}+\bar{W}_{2} \\
& \overline{\dot{Q}}=\frac{1}{\tau}\left(\int_{0}^{\tau} \dot{Q}(t) d t\right)=\frac{1}{\tau}\left(\int_{0}^{\tau / 2} \dot{Q}_{1}(t) d t+\int_{\tau / 2}^{\tau} \dot{Q}_{2}(t) d t\right)=\overline{\dot{Q}}_{1}+\overline{\dot{Q}}_{2}
\end{aligned}
$$

respectivamente. Podemos econtrar as expressões gerais para $\overline{\dot{W}}$ em função de forças arbitrárias $f_{1}(t)$ e $f_{2}(t)$. Tomando as expressões (4.1.8) e (4.1.9) e substituindo nas expressões (4.2.2), podemos escrever:

$$
\begin{aligned}
\bar{W}_{1}=-\frac{m}{\tau\left(e^{\gamma \tau}-1\right)} & {\left[\left(e^{\gamma \tau}-1\right) \int_{0}^{\tau / 2} e^{-\gamma t} f_{1}(t)\left(\int_{0}^{t} e^{\gamma t^{\prime}} f_{1}\left(t^{\prime}\right) d t^{\prime}\right) d t+\right.} \\
& \left.+\int_{0}^{\tau / 2} e^{\gamma t^{\prime}} f_{1}\left(t^{\prime}\right) d t^{\prime} \int_{0}^{\tau / 2} e^{-\gamma t} f_{1}(t) d t+e^{\gamma \tau / 2} \int_{\tau / 2}^{\tau} e^{\gamma\left(t^{\prime}-\tau / 2\right)} f_{2}\left(t^{\prime}\right) d t^{\prime} \int_{0}^{\tau / 2} e^{-\gamma t} f_{1}(t) d t\right]
\end{aligned}
$$

$$
\begin{aligned}
\overline{\dot{W}}_{2}=-\frac{m}{\tau\left(e^{\gamma \tau}-1\right)} & \left\{\left(e^{\gamma \tau}-1\right)\left[\int_{\tau / 2}^{\tau} f_{2}(t)\left(\int_{\tau / 2}^{t} f_{2}\left(t^{\prime}\right) d t^{\prime}\right) d t+e^{-\gamma / 2} \int_{0}^{\tau / 2} f_{1}\left(t^{\prime}\right) d t^{\prime} \int_{\tau / 2}^{\tau} f_{2}(t) d t\right]+\right. \\
& \left.+\int_{\tau / 2}^{\tau} e^{-\gamma(t-\tau / 2)} f_{2}(t) d t\left[e^{-\gamma \tau / 2} \int_{0}^{\tau / 2} e^{\gamma t^{\prime}} f_{1}\left(t^{\prime}\right) d t^{\prime}+\int_{\tau / 2}^{\tau} e^{\gamma\left(t^{\prime}-\tau / 2\right)} f_{2}\left(t^{\prime}\right) d t^{\prime}\right]\right\}
\end{aligned}
$$


A eficiência pode ser definida como a razão entre o trabalho útil extraído pela energia total fornecida. Assim, escrevemos:

$$
\eta=\frac{-P}{\overline{\bar{W}}_{1}+\overline{\dot{Q}}_{i}}
$$

onde a potência média $P$ é o fluxo de trabalho extraído no segundo reservatório, $P=\bar{W}_{2} \geq 0$. $\overline{\dot{Q}}_{i}$ é o calor médio cedido do reservatório ao sistema, que pode ser $\overline{\dot{Q}}_{1}$ ou $\overline{\dot{Q}}_{2}$ dependendo das temperaturas $T_{1}$ e $T_{2}$. É importante lembrarmos que o calor trocado depende de $\left\langle v_{i}^{2}(t)\right\rangle$ e $T_{i}$ (temperatura do reservatório) e o trabalho está associado à força externa e $\left\langle v_{i}(t)\right\rangle$. O reservatório não troca partículas com o sistema, de modo que, ao contrário da máquina de quantum dot, não há trabalho químico.

No caso em que as temperaturas são iguais, a eficiência é escrita como:

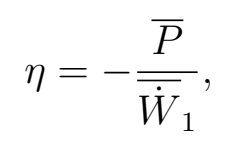

e o sistema se reduz a um "work-work converter"(conversor de um tipo de trabalho em outra forma de trabalho). Como feito na referência [25], essa expressão é ainda uma boa aproximação para o caso onde $\Delta \Gamma \approx 0$. A produção de entropia média calculada sobre um período é dada por:

$$
\bar{\Pi}=\frac{1}{\tau}\left(\int_{0}^{\tau} \Pi(t) d t\right)
$$

onde:

$$
\bar{\Pi}(\tau)=\frac{1}{\tau}\left(\int_{0}^{\tau} \Phi(t) d t\right)=\frac{1}{\tau}\left(\int_{0}^{\tau / 2} \Phi_{1}(t) d t+\int_{\tau / 2}^{\tau} \Phi_{2}(t) d t\right)=\bar{\Phi}_{1}+\bar{\Phi}_{2}
$$

que é como expresso na equação (2.6.44) como a razão entre o calor trocado e a temperatura:

$$
\bar{\Phi}_{i}=\frac{\overline{\dot{Q}}_{i}}{T_{i}}
$$

No estado estacionário, o valor da produção de entropia é dada por:

$$
\bar{\Pi}_{\mathrm{NESS}}=\bar{\Phi}_{\mathrm{NESS}}
$$

Podemos calcular o valor médio da produção de entropia, considerando a expressão geral para $\bar{Q}_{i}$ dada por (4.2.4). Para uma força genérica $f_{i}(t)=X_{i} g_{i}(t)$ na forma descrita por (4.1.12), podemos calcular a produção de entropia média. A expressão pode ser escrita em uma forma bilinear em termos dos drives e em função dos coeficientes de Onsager, conforme 
discutido na seção (2.2) e expresso na equação (2.1.9). Assim, podemos escrever a expressão acima como:

$$
\bar{\Pi}=L_{11} X_{1}^{2}+L_{22} X_{2}^{2}+\left(L_{12}+L_{21}\right) X_{1} X_{2}+L_{T T} f_{T}^{2}
$$

A expressão de $L_{T T}$ depende apenas da diferença de temperatura e é a mesma para qualquer força aplicada. Os coeficientes são dados por (considerando $\gamma=1$ e $k_{\mathrm{B}}=1$ ):

$$
\begin{aligned}
L_{11}=\frac{\csc ^{2}(\tau / 2)}{4 \Gamma_{2}}\left(\int_{0}^{\tau / 2} e^{t} g_{1}(t) d t\right)^{2}+\frac{2}{\tau \Gamma_{1}} & {\left[\int_{0}^{\tau / 2}\left(\int_{0}^{t} e^{t^{\prime}} g_{1}\left(t^{\prime}\right) d t^{\prime}\right)\left(\int_{0}^{t} e^{t^{\prime}} g_{1}\left(t^{\prime}\right) d t^{\prime}+2 e^{-\tau}\right) d t+\right.} \\
& \left.+\left(\int_{0}^{\tau / 2} e^{t^{\prime}-\tau} g_{1}\left(t^{\prime}\right) d t^{\prime}\right)^{2}\right] \\
L_{22}=\frac{1}{\Gamma_{1}}\left(\int_{\tau / 2}^{\tau} e^{t^{\prime}-\tau} g_{2}\left(t^{\prime}\right) d t^{\prime}\right)^{2}+\frac{1}{2 \tau \Gamma_{2}}\left[4 \int_{\tau / 2}^{\tau} \int_{\tau / 2}^{t} e^{t^{\prime}-\tau / 2} g_{2}\left(t^{\prime}\right) d t^{\prime}\left((\operatorname{coth}(\tau / 2)-1) \int_{t}^{\tau} e^{t^{\prime}-\tau / 2} g_{2}\left(t^{\prime}\right) d t^{\prime}\right) d t+\right. & \left.+(\operatorname{coth}(\tau / 2)-1)^{2}\left(\int_{\tau / 2}^{\tau} e^{t^{\prime}-\tau / 2} g_{2}\left(t^{\prime}\right) d t^{\prime}\right)^{2} \tau / 2\right]
\end{aligned}
$$

$$
\begin{aligned}
L_{12}=\frac{2}{\Gamma_{1} \tau} \int_{\tau / 2}^{\tau} e^{t^{\prime}-\tau / 2} g_{2}\left(t^{\prime}\right) d t^{\prime}\left(\int_{\tau / 2}^{t}\right. & \left.\int_{0}^{t} e^{t^{\prime}-\tau / 2} g_{1}\left(t^{\prime}\right) d t^{\prime} d t+\frac{\tau e^{-3 \tau / 2}}{2} \int_{0}^{\tau / 2} e^{t^{\prime}} g_{1}\left(t^{\prime}\right) d t^{\prime}\right)+ \\
+\frac{1}{4 \Gamma_{2} \tau} \int_{0}^{\tau / 2} e^{t^{\prime}} g_{1}\left(t^{\prime}\right) d t^{\prime} & \left(4 \int_{\tau / 2}^{\tau} \csc (\tau / 2) \int_{\tau / 2}^{t} e^{t^{\prime}-\tau / 2} g_{2}\left(t^{\prime}\right) d t^{\prime} d t+\right. \\
& \left.+\tau \int_{\tau / 2}^{\tau} e^{t^{\prime}-\tau / 2} g_{2}\left(t^{\prime}\right) d t^{\prime} \csc (\tau / 2)(\operatorname{coth}(\tau / 2)-1)\right)
\end{aligned}
$$

$$
\begin{aligned}
L_{21}=\frac{2}{\Gamma_{2} \tau} \int_{\tau / 2}^{\tau} e^{t^{\prime}-\tau / 2} g_{2}\left(t^{\prime}\right) d t^{\prime} & \left(\int_{\tau / 2}^{t} \int_{0}^{t} e^{t^{\prime}-\tau / 2} g_{1}\left(t^{\prime}\right) d t^{\prime} d t+\frac{\tau e^{-3 \tau / 2}}{2} \int_{0}^{\tau / 2} e^{t^{\prime}} g_{1}\left(t^{\prime}\right) d t^{\prime}\right)+ \\
+\frac{1}{4 \Gamma_{1} \tau} \int_{0}^{\tau / 2} e^{t^{\prime}} g_{1}\left(t^{\prime}\right) d t^{\prime} & \left(4 \int_{\tau / 2}^{\tau} \csc (\tau / 2) \int_{\tau / 2}^{t} e^{t^{\prime}-\tau / 2} g_{2}\left(t^{\prime}\right) d t^{\prime} d t+\right. \\
& \left.+\tau \int_{\tau / 2}^{\tau} e^{t^{\prime}-\tau / 2} g_{2}\left(t^{\prime}\right) d t^{\prime} \csc (\tau / 2)(\operatorname{coth}(\tau / 2)-1)\right)
\end{aligned}
$$

$$
\begin{gathered}
L_{T T}=\frac{\Gamma_{1} \Gamma_{2}}{2 \tau} \tanh \left(\frac{\tau}{2}\right), \\
f_{T}=\frac{1}{\Gamma_{2}}-\frac{1}{\Gamma_{1}} .
\end{gathered}
$$


A simetria dos coeficientes $L_{12}=L_{21}$ pode ou não ser válida, dependendo das forças $f_{1}(t)$ e $f_{2}(t)$. Para o caso polinomial aqui estudado na relação (4.2.55), essa simetria é sempre válida quando $\alpha=\beta$. Para o caso senoidal, a validade da simetria (ou eventual assimetria) depende do ângulo de defasagem $\phi$. Porém, as propriedades (2.2.6) sempre são válidas independente das forças externas. Simetrias relacionadas à troca $L_{11} \leftrightarrow L_{22}$ quando $\Gamma_{1} \leftrightarrow \Gamma_{2}$ também são observadas quando $\alpha=\beta$. Para o caso work-work converter, os coeficientes obtidos do trabalho coincidem com osobtidos da produção de entropia [25].

Distintos trabalhos abordam as condições em que sistemas colisionais como o modelado aqui operam como máquina [16,25,27-30]. Em geral, é necessária a existência de um fluxo termodinâmica externo ao sistema $J_{2} X_{2}<0$ contrabalanceando um fluxo $J_{1} X_{1}+L_{T T} f_{T}$. Em particular, para um caso onde as temperaturas dos reservatórios são iguais, o fluxo se opoe a $J_{1} X_{1}$. Fixando um valor para $X_{1}$, em geral o intervalo de valores de $X_{2}$ onde o sistema opera como máquina e portanto tem potência e eficiência maiores que zero e finitos corresponde aproximadamente ao intervalo $0 \leq\left|X_{2}\right| \leq\left|X_{2 m S}\right|$, onde $X_{2 m S}$ é o ponto de $X_{2}$ onde a produção de entropia média é mínima. Quando $L_{12}=L_{21}$, o valor o intervalo onde o sistema opera como máquina coincide exatamente ao intervalo acima.

A seguir, estudaremos o modelo colisional para dois reservatórios para vários exemplos de forças externas. Nosso objetivo é avançar os estudos em relação a referência [25], estudando a maximização da potência e da eficiência para diferentes drives. Para tal, vamos considerar os seguintes casos: forças constantes, polinomiais e trigonométricas. Inicialmente, recuperaremos alguns de seus principais resultados, começando com o caso de forças nulas, constates não nulas e polinomiais. Nosso estudo aqui é preliminar e algumas questões serão melhor respondidas em pesquisas futuras. 


\subsubsection{Ausência de Forças Externas}

Considerando o caso específico onde $f_{1}(t)=f_{2}(t)=0$, podemos reescrever as equações $(4.1 .6,4.1 .7)$, respectivamente para a velocidade e para a variância na forma:

$$
\begin{gathered}
\frac{d\left\langle v_{1}(t)\right\rangle}{d t}=-\gamma\left\langle v_{1}(t)\right\rangle, t \in[0, \tau / 2], \\
\frac{d\left\langle v_{2}(t)\right\rangle}{d t}=-\gamma\left\langle v_{2}(t)\right\rangle, t \in[\tau / 2, \tau], \\
\frac{d b_{1}(t)}{d t}=-2 \gamma b_{1}(t)+\Gamma_{1}, t \in[0, \tau / 2], \\
\frac{d b_{2}(t)}{d t}=-2 \gamma b_{2}(t)+\Gamma_{2}, t \in[\tau / 2, \tau] .
\end{gathered}
$$

Considerando as condições de continuidade, concluímos que $\left\langle v_{1}(t)\right\rangle=\left\langle v_{2}(t)\right\rangle=0$ e a variância repete os valores dados por $(4.1 .10,4.1 .11)$. Considerando que $b_{i}(t)=\left\langle v_{i}^{2}(t)\right\rangle-\left\langle v_{i}(t)\right\rangle^{2}$, escrevemos, para cada reservatório:

$$
\begin{aligned}
& \left\langle v_{1}^{2}(t)\right\rangle=\frac{\Gamma_{1}}{2 \gamma}+\frac{\Gamma_{2}-\Gamma_{1}}{2 \gamma\left(1+e^{\gamma \tau}\right)} e^{\gamma / 2(-t+\tau / 2)} ; t \in[0, \tau / 2], \\
& \left\langle v_{2}^{2}(t)\right\rangle=\frac{\Gamma_{2}}{2 \gamma}-\frac{\Gamma_{2}-\Gamma_{1}}{2 \gamma\left(1+e^{\gamma \tau}\right)} e^{-2 \gamma(t-\tau / 2)+\gamma \tau} ; t \in[\tau / 2, \tau] .
\end{aligned}
$$

Pela expressão (4.2.10), podemos encontrar a produção de entropia, com:

$$
\begin{aligned}
& \bar{\Phi}_{1}=\frac{1}{\tau T_{i}}\left(\int_{0}^{\tau / 2} \dot{Q}_{1}(t) d t\right), \\
& \bar{\Phi}_{2}=\frac{1}{\tau T_{i}}\left(\int_{\tau / 2}^{\tau} \dot{Q}_{2}(t) d t\right),
\end{aligned}
$$

onde $\dot{Q}_{i}(t)$ é dada pelas expressões (4.2.2). Assim, para o caso sem forças externas, temos:

$$
\bar{\Pi}(\tau)=\frac{\Gamma_{1} \Gamma_{2}}{2 \tau}\left(\frac{1}{\Gamma_{1}}-\frac{1}{\Gamma_{2}}\right)^{2} \tanh \left(\frac{\gamma \tau}{2}\right) .
$$

A seguir, exibimos o gráfico da expressão (4.2.28) em relação a $\tau$, fixando a temperatura do reservatório frio para $\Gamma_{2}=1$. 


\section{CAPÍTULO 4. MODELO COLISIONAL PARA UMA PARTÍCULA BROWNIANA}

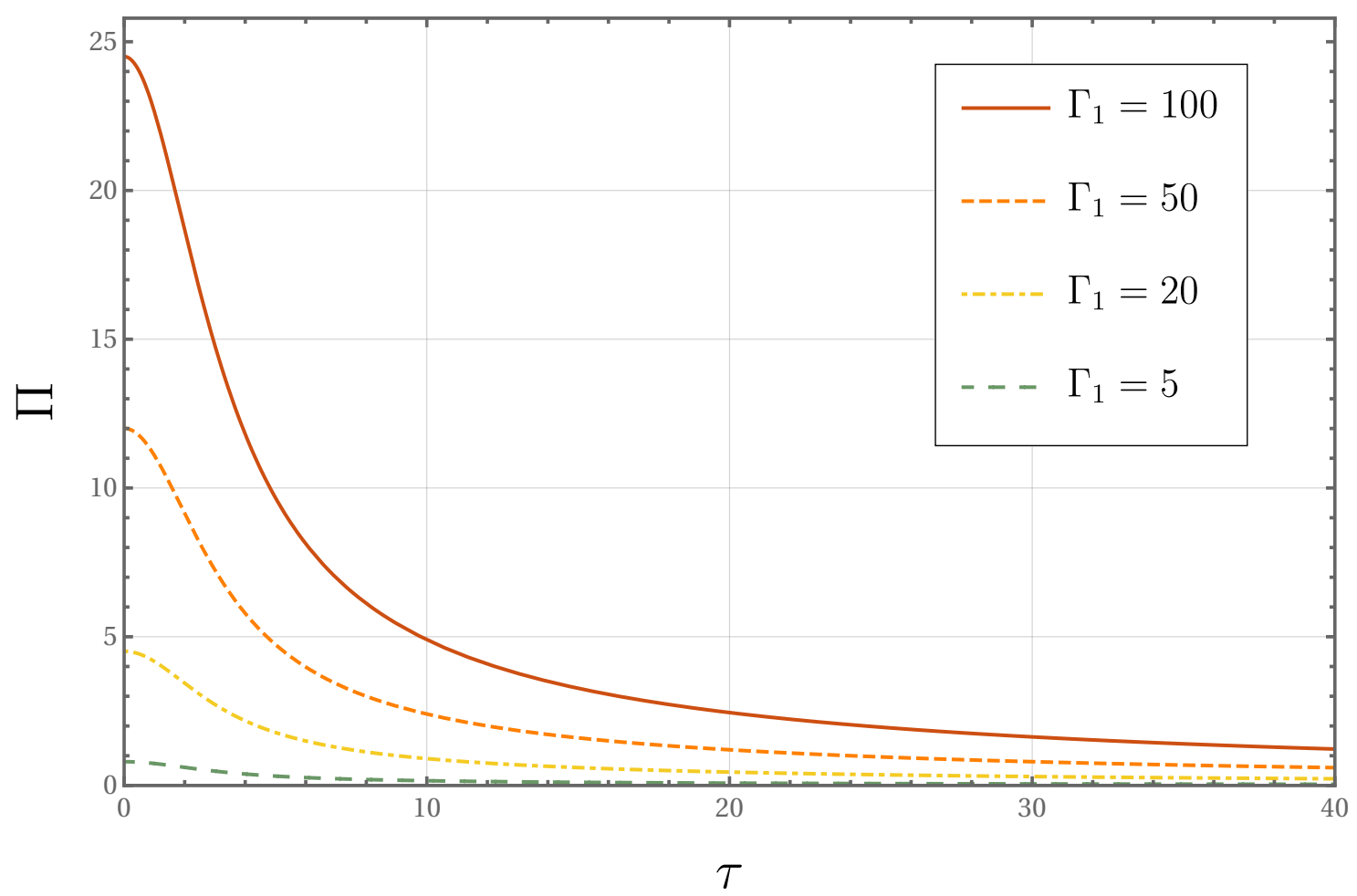

Figura 4.1: Produção de entropia pelo período $\tau$ para diferentes temperaturas. Fixamos $k_{\mathrm{B}}=1, \gamma=1$ e $\Gamma_{2}=1$

Como discutimos anteriormente, podemos escrever a produção de entropia em termos dos coeficientes de Onsager, como na forma geral (4.2.13). Na expressão (4.2.28), reconhecemos explicitamente a força associada a grandeza intensiva $(1 / \Gamma), f_{\mathrm{T}}$ dada por:

$$
f_{\mathrm{T}}=\frac{1}{\Gamma_{2}}-\frac{1}{\Gamma_{1}} .
$$

Da expressão (4.2.28), escrevemos:

$$
\bar{\Pi}=L_{\mathrm{TT}} f_{\mathrm{T}}^{2},
$$

com:

$$
L_{\mathrm{TT}}=\frac{\Gamma_{1} \Gamma_{2}}{2 \tau} \tanh \left(\frac{\gamma \tau}{2}\right) .
$$

O plot da grandeza $L_{\mathrm{TT}}$ para diferentes temperaturas é dado a seguir: 


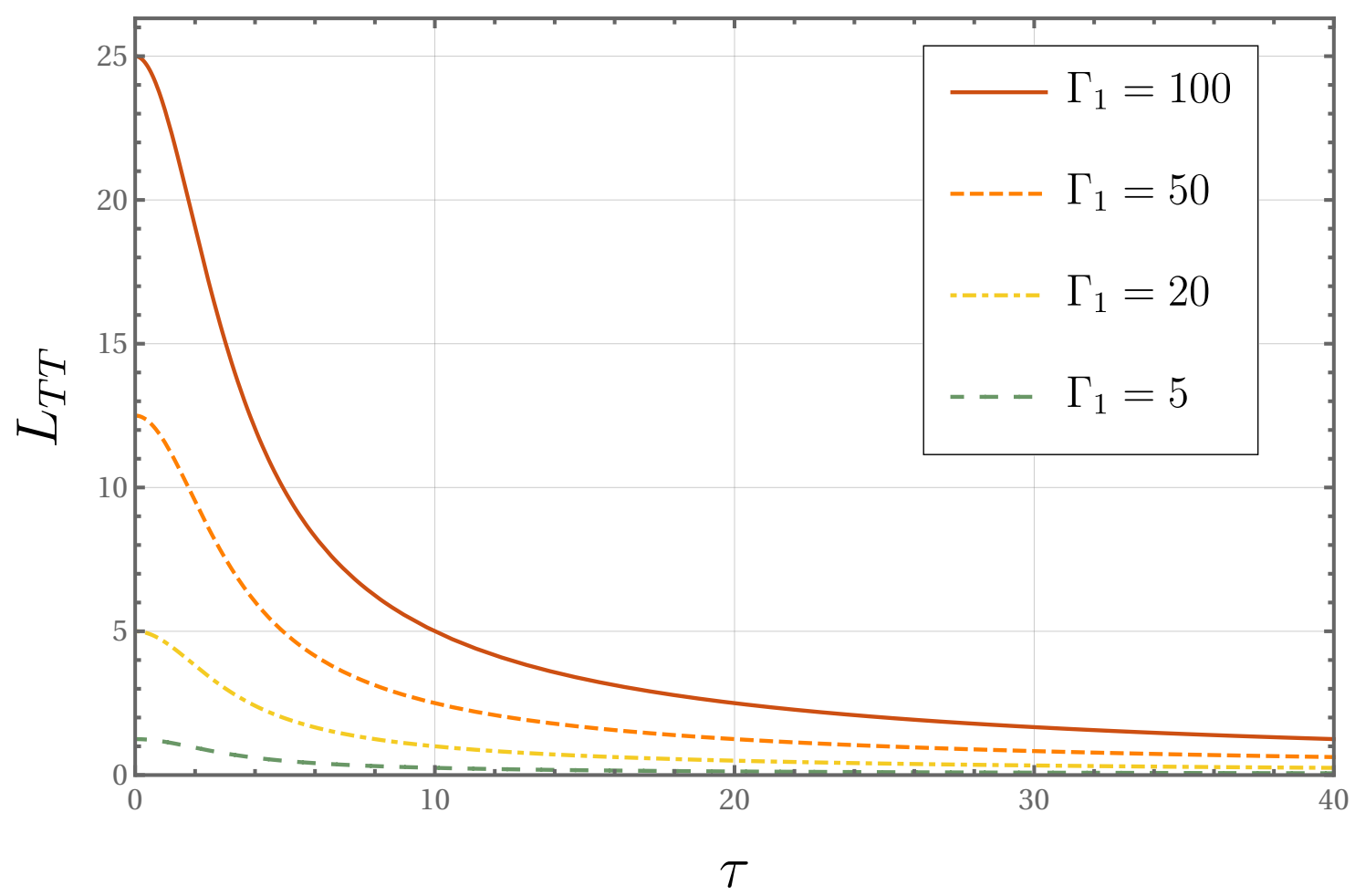

Figura 4.2: $L_{\text {TT }}$ pelo período $\tau$ para diferentes temperaturas. Fixamos $k_{\mathrm{B}}=1, \gamma=1 \mathrm{e}$ $\Gamma_{2}=1$

Tanto o coeficiente de Onsager $L_{\mathrm{TT}}$ e a produção de entropia $\bar{\Pi}$ são grandezas não negativas, como esperado. Em particular, a produção de entropia se anula quando $\Gamma_{1}=\Gamma_{2}$. Na ausência de forças externas, o sistema não realiza trabalho e portanto, a potência média extraída do sistema é nulo. Toda a energia extraída do reservatório quente em forma de fluxo de calor é doada ao reservatório frio, sem dissipações. Os resultados acima reproduzem os da referência [25] para o caso onde há ausências de forças externas.

\subsubsection{Forças Constantes}

Agora, consideraremos em cada reservatório $i$ seja exercida uma força constante $X_{i}$, como descrito a seguir:

$$
f(t)=\left\{\begin{array}{l}
f_{1}(t)=X_{1}, t \in[0, \tau / 2], \\
f_{2}(t)=X_{2}, t \in[\tau / 2, \tau] .
\end{array}\right.
$$


Para cada reservatório, podemos escrever a evolução temporal das velocidades médias como:

$$
\begin{aligned}
& \frac{d\left\langle v_{1}(t)\right\rangle}{d t}=-\gamma\left\langle v_{1}(t)\right\rangle+X_{1}, t \in[0, \tau / 2], \\
& \frac{d\left\langle v_{2}(t)\right\rangle}{d t}=-\gamma\left\langle v_{2}(t)\right\rangle+X_{2}, t \in[\tau / 2, \tau] .
\end{aligned}
$$

As variâncias de cada reservatório são dadas pelas equações (4.1.10, 4.1.11). Considerando as mesmas condições de contorno para garantir a continuidade da distribuição de probabilidade $\left(\left\langle v_{1}(\tau / 2)\right\rangle=\left\langle v_{2}(\tau / 2)\right\rangle\right.$ e $\left.b_{1}(\tau / 2)=b_{2}(\tau / 2)\right)$. Assim, escrevemos:

$$
\begin{aligned}
& \left\langle v_{1}(t)\right\rangle=\frac{e^{-t \gamma}}{\gamma}\left(\left(e^{t \gamma}-1\right) X_{1}+\frac{X_{1}+X_{2} e^{\gamma \tau / 2}}{1+e^{\gamma \tau / 2}}\right) \\
& \left\langle v_{2}(t)\right\rangle=\frac{e^{-\gamma(t-\tau / 2)}}{\gamma}\left[e^{-\gamma \tau / 2}\left(\left(e^{\gamma \tau / 2}-1\right) X_{1}+\frac{X_{1}+X_{2} e^{\gamma \tau / 2}}{1+e^{\gamma \tau / 2}}\right)+\left(e^{t \gamma-\gamma \tau / 2}-1\right) X_{2}\right] .
\end{aligned}
$$

Com base no valor das variâncias de cada reservatório dadas pelas expressões $(4.1 .10,4.1 .11)$ :

$$
\begin{aligned}
& \left\langle v_{1}^{2}(t)\right\rangle=\frac{e^{-2 t \gamma}}{\gamma^{2}}\left[\left(e^{t \gamma}-1\right) X_{1}+\frac{X_{1}-X_{2}}{1+e^{\gamma \tau / 2}}+X_{2}\right]^{2}+\frac{e^{\gamma(\tau-2 t)}\left(\Gamma_{2}-\Gamma_{1}\right)}{2 \gamma\left(1+e^{\tau \gamma}\right)}+\frac{\Gamma_{1}}{2 \gamma}, \\
& \left\langle v_{2}^{2}(t)\right\rangle=\frac{e^{-2 t \gamma}}{\gamma^{2}}\left[\frac{e^{\gamma \tau}\left(X_{1}-X_{2}\right)+e^{t \gamma}\left(1+e^{\gamma \tau / 2}\right) X_{2}}{1+e^{\gamma \tau / 2}}\right]^{2}+\frac{e^{2 \gamma(\tau-t)}\left(\Gamma_{1}-\Gamma_{2}\right)}{2 \gamma\left(1+e^{\gamma \tau}\right)}+\frac{\Gamma_{2}}{2 \gamma} .
\end{aligned}
$$

Inicialmente, analisaremos a eficiência para o caso onde os dois reservatórios têm a mesma temperatura, $\Gamma_{1}=\Gamma_{2}$. Nesse caso, conforme mencionamos anteriormente a eficiência é dada pela expressão:

$$
\eta=\frac{-P}{\overline{\dot{W}}_{1}} .
$$

Onde a potência média $P=\bar{W}_{2} \geq$. Podemos expressar a o valor exato para os trabalhos médios, tomando as formas gerais dadas por $(4.2 .5,4.2 .6)$, fazendo $m=1$ e $\gamma=1$ :

$$
\begin{aligned}
& \overline{\dot{W}}_{1}=-\frac{X_{1}\left(X_{1} \tau+2\left(X_{2}-X_{1}\right) \tanh (\tau / 4)\right)}{2 \tau}, \\
& \overline{\dot{W}}_{2}=-\frac{X_{2}\left(X_{2} \tau+2\left(X_{1}-X_{2}\right) \tanh (\tau / 4)\right)}{2 \tau} .
\end{aligned}
$$

A partir daí, considerando a expressão geral da eficiência, escrevemos:

$$
\eta=-\frac{X_{2}\left(X_{2} \gamma \tau+2\left(X_{1}-X_{2}\right) \tanh (\gamma \tau / 4)\right)}{X_{1}\left(X_{1} \gamma \tau+2\left(X_{2}-X_{1}\right) \tanh (\gamma \tau / 4)\right)}
$$

Fixando $X_{1}=1$, devemos encontrar o intervalo de valores de $X_{2}$ onde o sistema opera como uma máquina, isto é: a potência média é positiva e valor da eficiência positiva e 
finita. Este regime pode ser dado pelo valor de $X_{2}$ onde a produção de entropia em função de $X_{2}$ seja mínima até $X_{2}=0,\left[-\left|X_{2 m S}\right|, 0\right]$. Isso é verdade quando há simetria nos coeficientes $L_{12}=L_{21}$ [25]. A seguir, o gráfico da eficiência é exibido em função dos valores de $X_{2}$ pertecentes a esse intervalo. Com o objetivo de se maximizar potência e eficiência da máquina neste regime, as grandezas $X_{2 m S}, X_{2 m E}$ e $X_{m P}$ que representam os valores de $X_{2}$ que, respectivamente, minimizam a entropia, maximizam a eficiência e a potência têm fundamental importância. Uma vez encontrada as expressões da eficiência, entropia e potência, podemos encontrar essas grandezas de maneira direta.

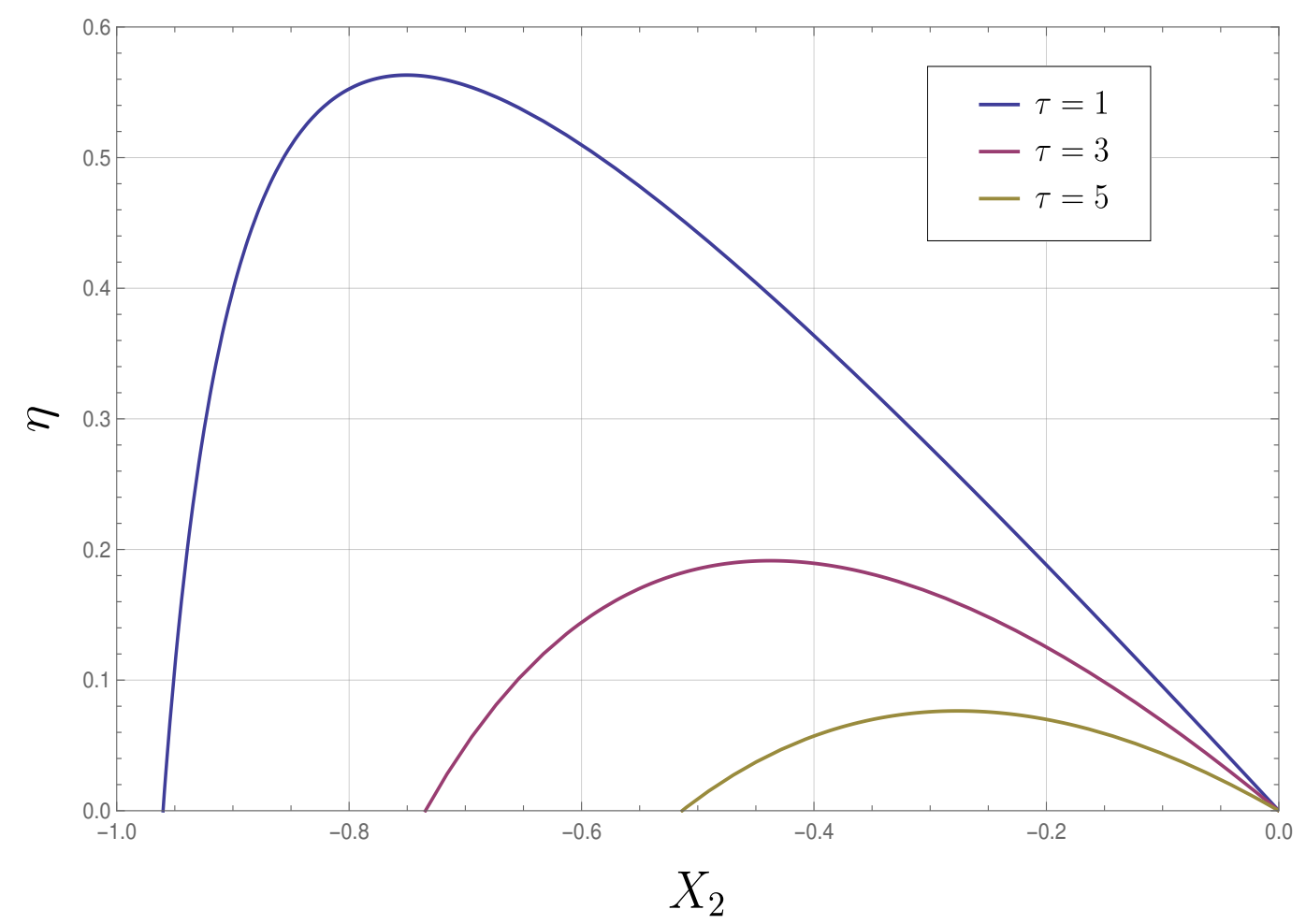

Figura 4.3: Eficiência em relação a $X_{2}$, fixando em $X_{1}=1$ para alguns valores de períodos $\tau$, para $\Gamma_{1}=\Gamma_{2}=2$.

Nesse mesmo intervalo, a máquina deve ter a potência média $P$ positiva. A seguir, apresentamos as curvas da expressão (4.2.41) variando $\tau$. Observamos que, ao aumentar o intervalo de tempo do período da máquina, há uma queda significativa da potência e da eficiência. 


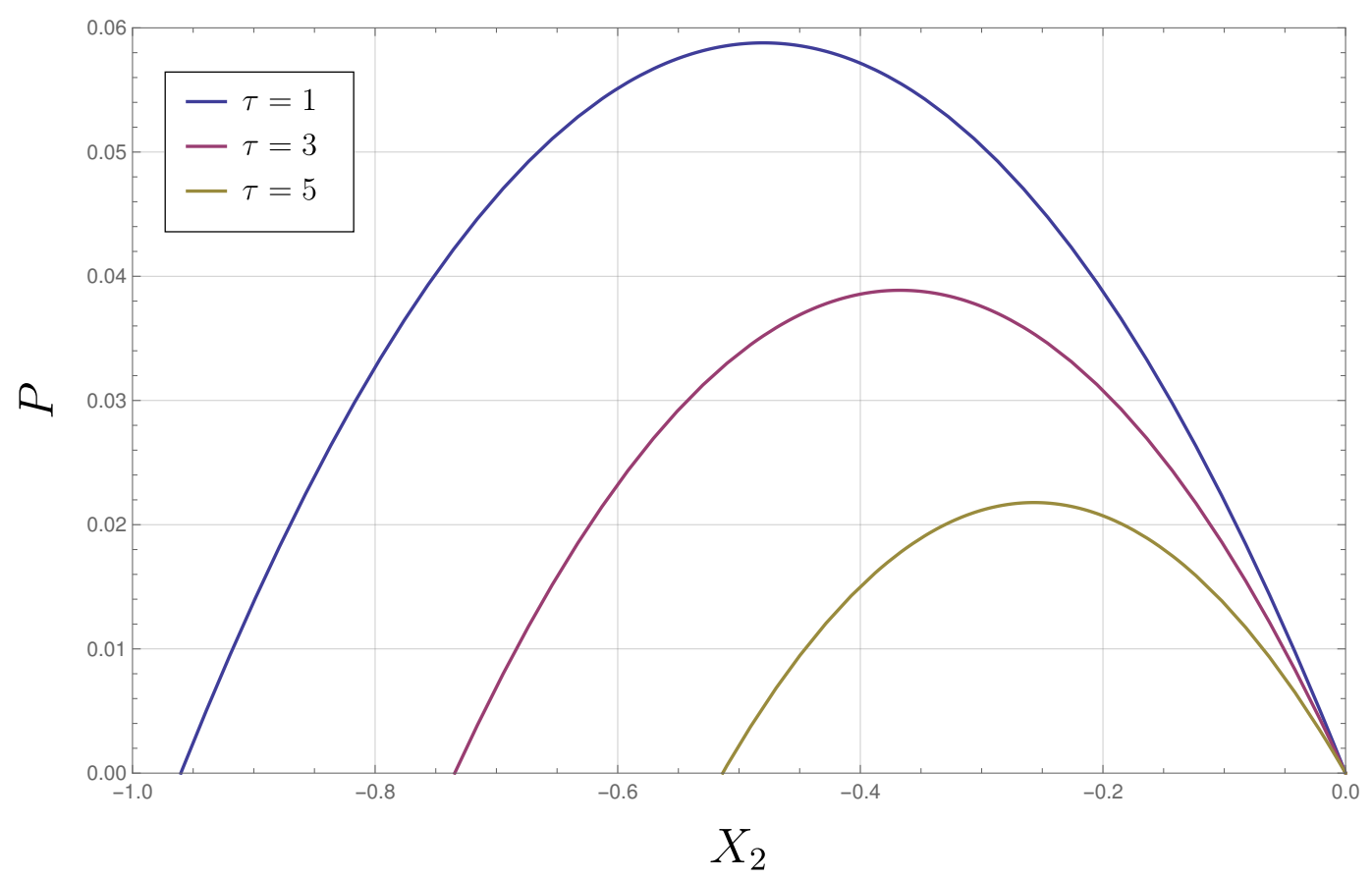

Figura 4.4: Potência $P$ em função da força $X_{2}$ para alguns valores de $\tau$, fixando $X_{1}=1$, e $\Gamma_{1}=\Gamma_{2}=2$.

Podemos escrever a produção de entropia média do sistema usando a expressão (4.2.10), de forma que, tomando $m=1$ e $\gamma=1$ :

$$
\begin{aligned}
\bar{\Pi}=\frac{1}{\tau}[ & X_{1}^{2}\left(\frac{\Gamma_{1} \tau+\left(\Gamma_{2}-3 \Gamma_{1}\right) \tanh (\tau / 4)}{\Gamma_{1} \Gamma_{2}}\right)+X_{2}^{2}\left(\frac{\Gamma_{2} \tau+\left(\Gamma_{1}-3 \Gamma_{2}\right) \tanh (\tau / 4)}{\Gamma_{1} \Gamma_{2}}\right)+ \\
& \left.+2 X_{1} X_{2}\left(\frac{1}{\Gamma_{1}}+\frac{1}{\Gamma_{2}}\right) \tanh (\tau / 4)+\frac{\Gamma_{1} \Gamma_{2}}{2 \tau} \tanh (\tau / 2)\left(\frac{1}{\Gamma_{2}}-\frac{1}{\Gamma_{1}}\right)^{2}\right]
\end{aligned}
$$

onde:

$$
\begin{aligned}
f_{T} & =\frac{1}{\Gamma_{2}}-\frac{1}{\Gamma_{1}}, \\
L_{T T} & =\frac{\Gamma_{1} \Gamma_{2}}{2 \tau} \tanh (\tau / 2),
\end{aligned}
$$

e os coeficientes $L_{11}$ e $L_{12}$ são dados por:

$$
\begin{aligned}
& L_{11}=\frac{\Gamma_{1} \tau+\left(\Gamma_{2}-3 \Gamma_{1}\right) \tanh (\tau / 4)}{\Gamma_{1} \Gamma_{2}}, \\
& L_{12}=\left(\frac{1}{\Gamma_{1}}+\frac{1}{\Gamma_{2}}\right) \tanh (\tau / 4) .
\end{aligned}
$$


Os coeficientes $L_{22}$ e $L_{21}$ são obtidos substituindo respectivamente nas expressões (4.2.46, 4.2.47) $\Gamma_{1} \leftrightarrow \Gamma_{2}$. O coeficiente $L_{T T}$ é não negativo e os coeficientes $L_{i j}$ devem obedecer as propriedades (2.2.6).

A expressão da eficiência (4.2.42) pode ser melhor escrito em termos dos coeficientes de Onsager:

$$
\eta=-\frac{L_{22} X_{2}^{2}+L_{21} X_{1} X_{2}}{L_{12} X_{1} X_{2}+L_{11} X_{1}^{2}}
$$

que é uma expressão válida para $\Delta \Gamma=0$ [16], onde $L_{12}$ são escritas como funções dos parâmetros que descrevem o drive. Fixando $X_{1}=1$, podemos encontrar o ponto onde a eficiência é máxima, $X_{2 m E}$, por meio da seguinte equação:

$$
\left.\frac{\partial \eta}{\partial X_{2}}\right|_{X_{2}=X_{2 m E}}=0
$$

Considerando $X_{1}=1$ e dada a simetria $L_{12}=L_{21}$ :

$$
X_{2 m E}=-\frac{L_{11} L_{22}+\sqrt{L_{11} L_{22}\left(L_{11} L_{22}-L_{12}^{2}\right)}}{L_{12} L_{22}}
$$

A seguir, apresentamos os gráficos da produção de entropia. A região das curvas contínuas representam o intervalo de $X_{2}$ onde o sistema opera como máquina. Os pontos representam os valores de mínima entropia, correspondentes aos valores de $X_{2 m S}$ para cada ciclo. 


\section{CAPÍTULO 4. MODELO COLISIONAL PARA UMA PARTÍCULA BROWNIANA}

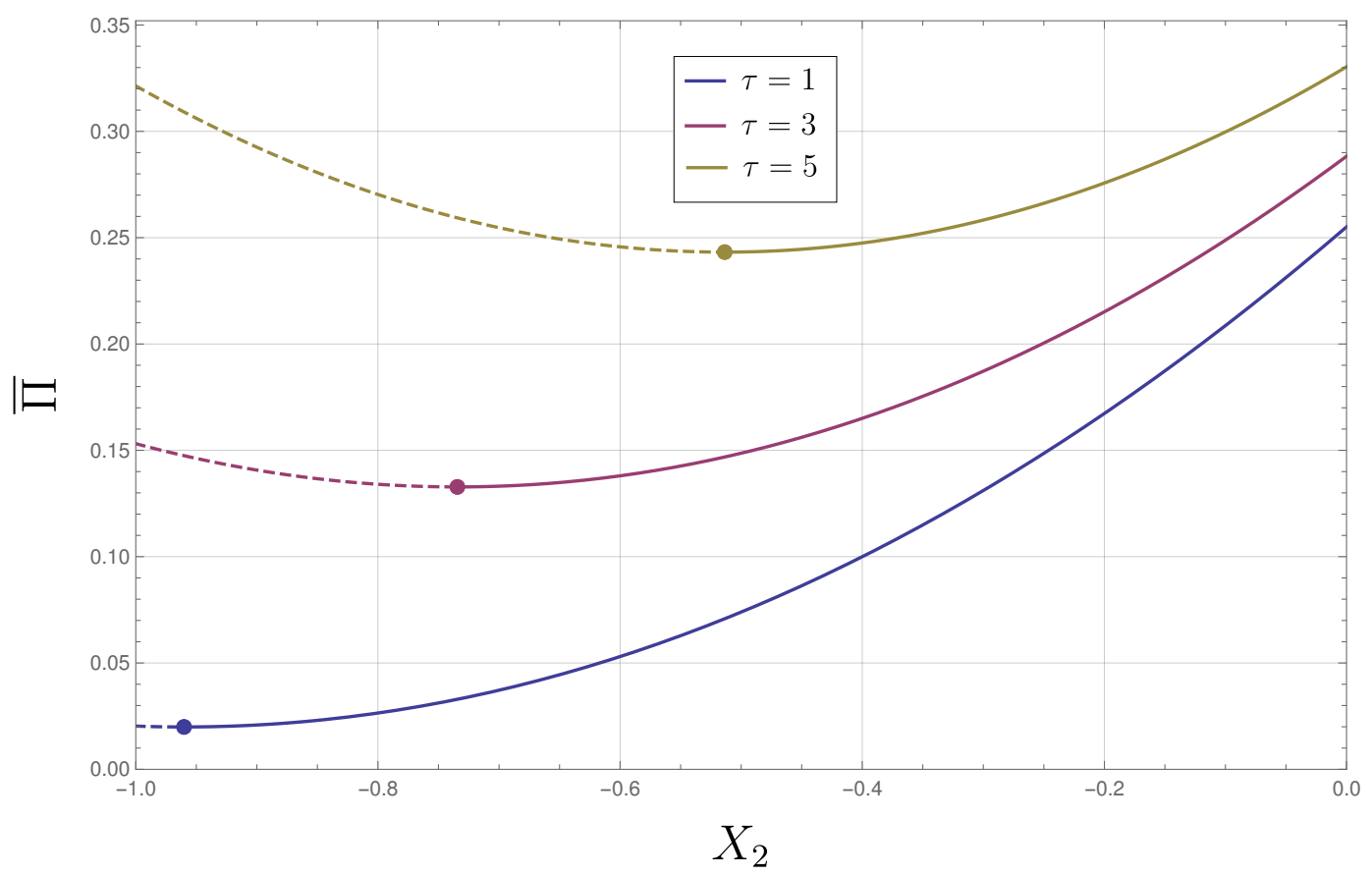

Figura 4.5: Produção de entropia $\bar{\Pi}$ em função da força $X_{2}$ para alguns valores de $\tau$, fixando $X_{1}=1$, e $\Gamma_{1}=\Gamma_{2}=2$. Os pontos representam o mínimo da produção de entropia. As linhas contínuas representam o intervalo onde o ciclo funciona como máquina (potência útil extraída positiva).

Outra grandeza fundamental a ser estudada nesse tipo de sistema é a eficiência em máxima potência. Dada a expressão exata da potência calculada em (4.4), podemos encontrar $X_{2 m P}$ partindo da equação:

$$
\left.\frac{\partial P}{\partial X_{2}}\right|_{X_{2}=X_{2 m P}}=\left.\frac{\partial \overline{\dot{W}}_{2}}{\partial X_{2}}\right|_{X_{2}=X_{2 m P}}=0
$$

Esse ponto é exatamente igual a:

$$
X_{2 m P}=-\frac{X_{1} \tanh (\tau / 4)}{\tau-2 \tanh (\tau / 4)} .
$$

Substituindo esse ponto na expressão da eficiência, para $X_{1}=1$, temos a eficiência em máxima potência $\eta_{m P}$ dada por:

$$
\eta_{m P}=\frac{2 \sinh ^{2}(\tau / 4)}{-2+\tau^{2}+\left(2+\tau^{2}\right) \cosh (\tau / 2)-4 \tau \sinh (\tau / 2)}
$$

A potência maximizada é dada por $P_{m P}$. Ambas as grandezas variam em relação ao período do ciclo, como expresso na figura abaixo: 


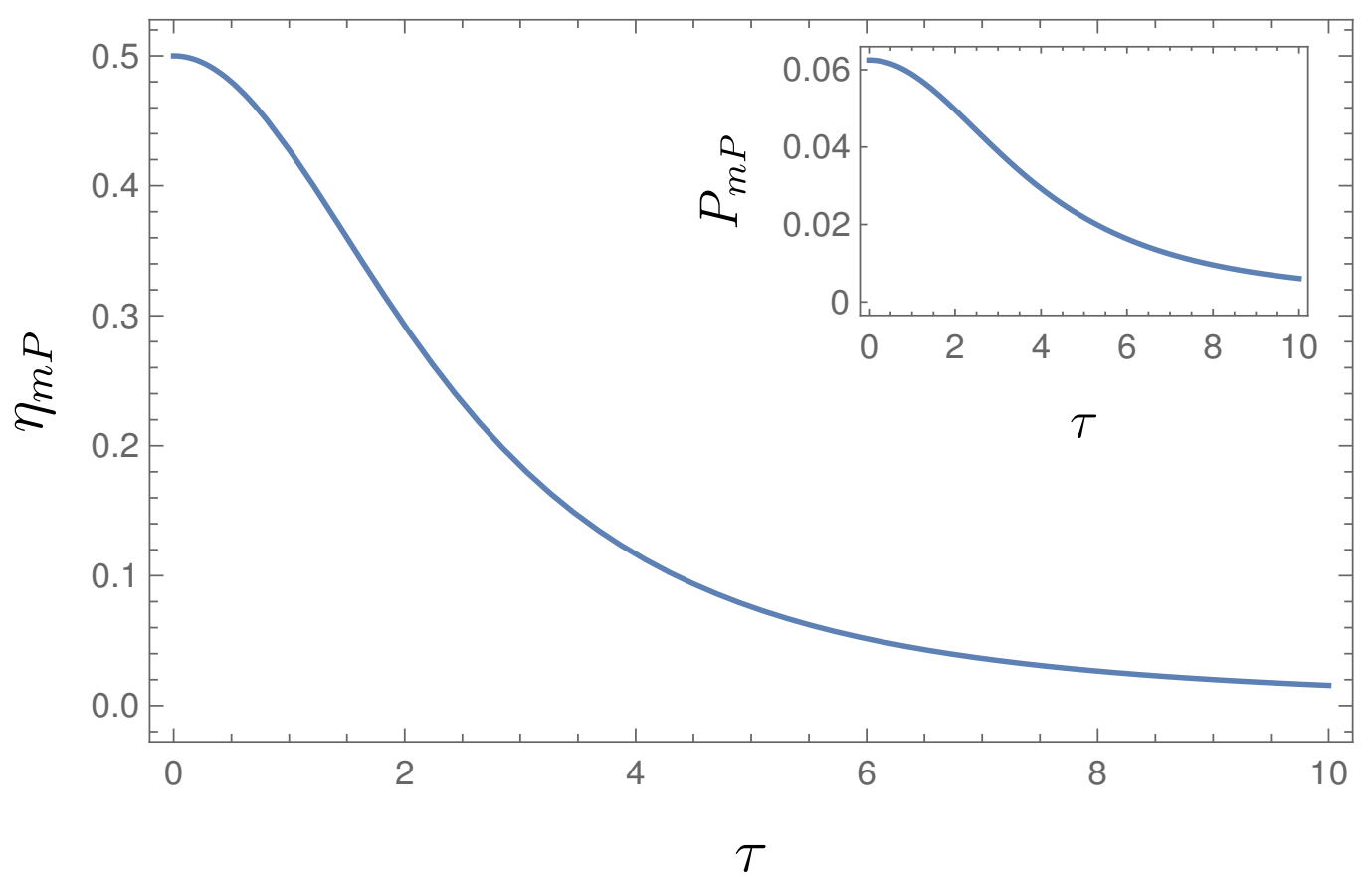

Figura 4.6: Curvas da eficiência em máxima potência $\eta_{m P}$ e da potência máxima $P_{m P} \mathrm{em}$ relação a $\tau$ para $\Gamma_{1}=\Gamma_{2}=2$ e fixando $X_{1}=1$.

Notamos que para o regime de ciclos rápidos, $\tau<<1, \eta_{m P} \rightarrow 1 / 2$, resultado já expressado na referência [25]. A expressão da potência nesse mesmo regime é dada por:

$$
P_{m P} \rightarrow \frac{X_{1}^{2}}{16}
$$

\subsubsection{Forças Polinomiais}

A seguir, estudaremos um caso geral para a força na forma discutida na seção (4.2.2), considerando uma forma polinomial, como descrita a seguir:

$$
f(t)=\left\{\begin{array}{l}
f_{1}(t)=X_{1}\left(\frac{2 t}{\tau}\right)^{\alpha}, t \in[0, \tau / 2], \\
f_{2}(t)=X_{2}\left(1-\frac{2 t}{\tau}\right)^{\beta}, t \in[\tau / 2, \tau] .
\end{array}\right.
$$

A nível deste estudo e por simplicidade, consideraremos que $\alpha$ e $\beta$ são inteiros não negativos. O caso da força constante discutido na seção (4.2.2) é reobtida tomando $\alpha=\beta=0$. Faremos aqui uma análise numérica e mais geral da influência dos fatores $\alpha$ e $\beta$ na eficiência, potência 


\section{CAPÍTULO 4. MODELO COLISIONAL PARA UMA PARTÍCULA BROWNIANA}

e na produção de entropia. É importante enfatizar que a análise para este caso feita aqui é preliminar e introdutória, generalizando os resultados obtidos pela referência [25].

Mais uma vez, consideraremos que $\Gamma_{1}=\Gamma_{2}$, com a eficiência dada pela expressão (4.2.39). Podemos encontrar a expressão geral para $\bar{W}_{1}$ e $\bar{W}_{2}$ pelas expressões $(4.2 .5,4.2 .6)$.

$$
\begin{aligned}
& \overline{\dot{W}}_{1}=-\frac{1}{\tau\left(e^{\gamma \tau}-1\right)}\left[\left(e^{\gamma \tau}-1\right) X_{1}^{2} \int_{0}^{\tau / 2} e^{-\gamma t}\left(\frac{2 t}{\tau}\right)^{\alpha}\left(\int_{0}^{t} e^{\gamma t^{\prime}}\left(\frac{2 t^{\prime}}{\tau}\right)^{\alpha} d t^{\prime}\right) d t+\right. \\
& \left.X_{1}^{2} \int_{0}^{\tau / 2} e^{\gamma t^{\prime}}\left(\frac{2 t^{\prime}}{\tau}\right)^{\alpha} d t^{\prime} \int_{0}^{\tau / 2} e^{-\gamma t}\left(\frac{2 t}{\tau}\right)^{\alpha} d t+e^{\gamma \tau / 2} X_{1} X_{2} \int_{\tau / 2}^{\tau} e^{\gamma\left(t^{\prime}-\tau / 2\right)}\left(1-\frac{2 t^{\prime}}{\tau}\right)^{\beta} d t^{\prime} \int_{0}^{\tau / 2} e^{-\gamma t}\left(\frac{2 t}{\tau}\right)^{\alpha} d t\right]
\end{aligned}
$$

$$
\begin{aligned}
\bar{W}_{2} & =-\frac{1}{\tau\left(e^{\gamma \tau}-1\right)}\left\{( e ^ { \gamma \tau } - 1 ) \left[X_{2}^{2} \int_{\tau / 2}^{\tau} e^{-\gamma(t-\tau / 2)}\left(1-\frac{2 t}{\tau}\right)^{\beta}\left(\int_{\tau / 2}^{\tau} e^{\gamma\left(t^{\prime}-\tau / 2\right)}\left(1-\frac{2 t^{\prime}}{\tau}\right)^{\beta} d t^{\prime}\right) d t+\right.\right. \\
& \left.e^{-\gamma \tau / 2} X_{1} X_{2} \int_{0}^{\tau / 2} e^{\gamma t^{\prime}}\left(\frac{2 t^{\prime}}{\tau}\right)^{\alpha} d t^{\prime} \int_{\tau / 2}^{\tau} e^{-\gamma(t-\tau / 2)}\left(1-\frac{2 t}{\tau}\right)^{\beta} d t\right]+X_{2}\left[X_{1} \int_{0}^{\tau / 2} e^{\gamma\left(t^{\prime}-\gamma \tau / 2\right)}\left(\frac{2 t^{\prime}}{\tau}\right)^{\alpha} d t^{\prime}+\right. \\
& \left.\left.X_{2} \int_{\tau / 2}^{\tau} e^{\gamma\left(t^{\prime}-\tau / 2\right)}\left(1-\frac{2 t^{\prime}}{\tau}\right)^{\beta} d t^{\prime}\right] \int_{\tau / 2}^{\tau} e^{-\gamma(t-\tau / 2)}\left(1-\frac{2 t}{\tau}\right)^{\beta} d t\right\}
\end{aligned}
$$

A solução completa da expressão acima, para $\alpha$ e $\beta$ inteiros positivos, envolve a manipulação de funções Gamma e funções Gamma incompletas. Por simplicidade, neste estudo faremos uma análise numérica na potência e eficiência para alguns valores de $\alpha$ e $\beta$ inteiros.

Com as temperaturas iguais, temos novamente um sistema do tipo "work-work converter", cuja eficiência é dada novamente pela expressão:

$$
\eta=-\frac{\overline{\dot{W}}_{2}}{\overline{\dot{W}}_{1}} .
$$

Para a nossa análise, considerarmos um valor inteiro fixo para $\beta$, estudando a variação dos comportamentos com a escolha de $\alpha$. Considere inicialmente $\beta=0$ e tomando $\alpha \in\{0,1,2\}$. Para $\alpha=0$, retornamos ao caso estudado na seção (4.2.2). A seguir, exibimos as curvas para a eficiência e a potência: 

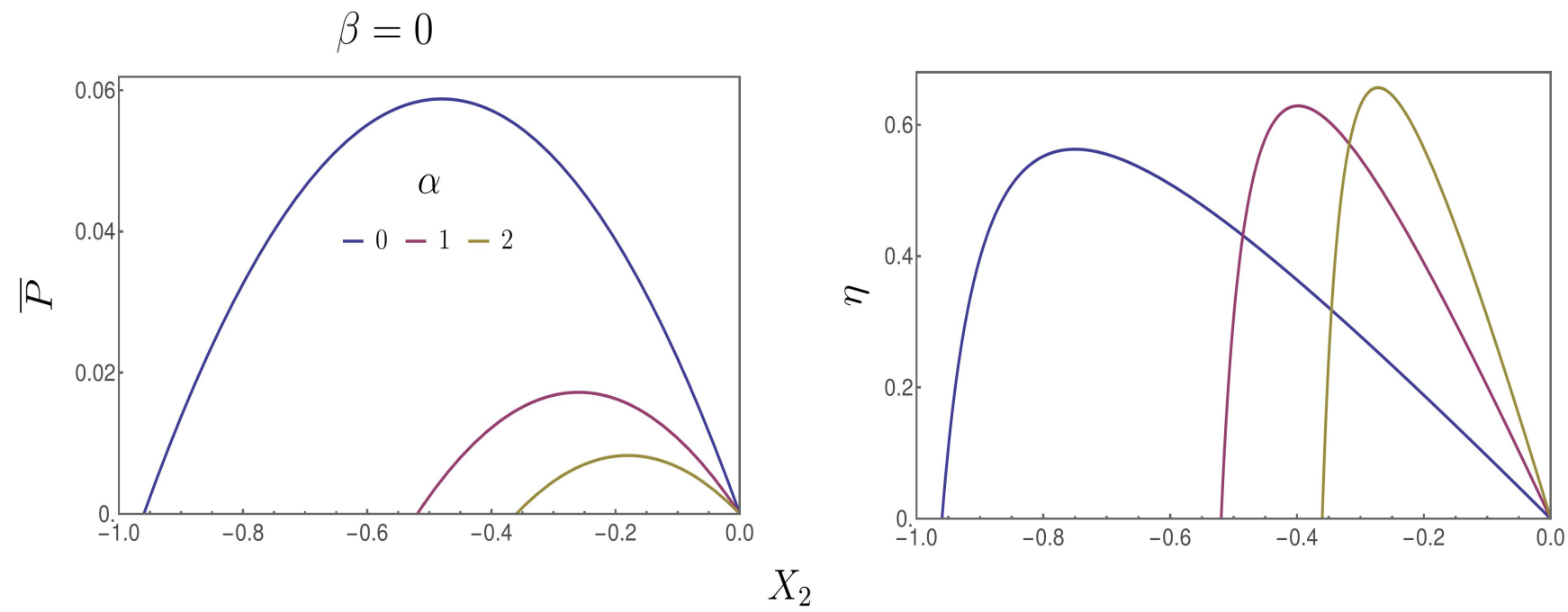

Figura 4.7: Eficiência $\eta$ e potência $\bar{P}$ em função da força $X_{2}$ para três valores de $\alpha$, fixando $\beta=0, X_{1}=1, \tau=1$ e $\beta=0$.

Primeiro, notamos um ganho na eficiência da máquina ao aumentar $\alpha$ em comparação com o caso constante $(\alpha=\beta=0)$. Por outro lado, o intervalo de valores de $X_{2}$ onde o sistema opera como máquina diminui. Em outras palavras, por mais que melhoramos a performance da máquina para $\beta=0$ ao aumentar $\alpha$, diminuimos o intervalo de força onde a potência útil é extraída. Além disso, há uma diminuição significativa da potência da máquina, como expresso na Fig. (4.7).

A diminuição no intervalo de valores $X_{2}$ onde o sistema opera como máquina pode ser estudada pela análise da produção de entropia. A seguir, exibimos a variação da produção de entropia média do sistema $\bar{\Pi}$, calculada pela expressão (4.2.9), em termos de $X_{2}$. A região de linhas contínuas expressa a região onde o sistema opera como máquina para cada valor de $\alpha$ e $\beta$ e o ponto, o valor mínimo de $\Pi$ em relação a $X_{2}$. Podemos observar que, como discutimos para o caso de forças constantes, o sistema opera como máquina aproximadamente no intervalo $\left[-\left|X_{2 m S}\right|, 0\right]$. Mais precisamente, esse intervalo descreve exatamente o regime de máquina para o caso $\alpha=\beta=0$. Para outros valores, o ponto de mínima entropia não corresponde exatamente ao intervalo onde sistema opera como máquina. Isso se deve ao fato da simetria dos coeficientes $L_{12}$ e $L_{21}$ não ser verificado. Mesmo assim, ainda é uma boa estimativa para a condição de $X_{2}$ para o sistema gerar potência útil. 


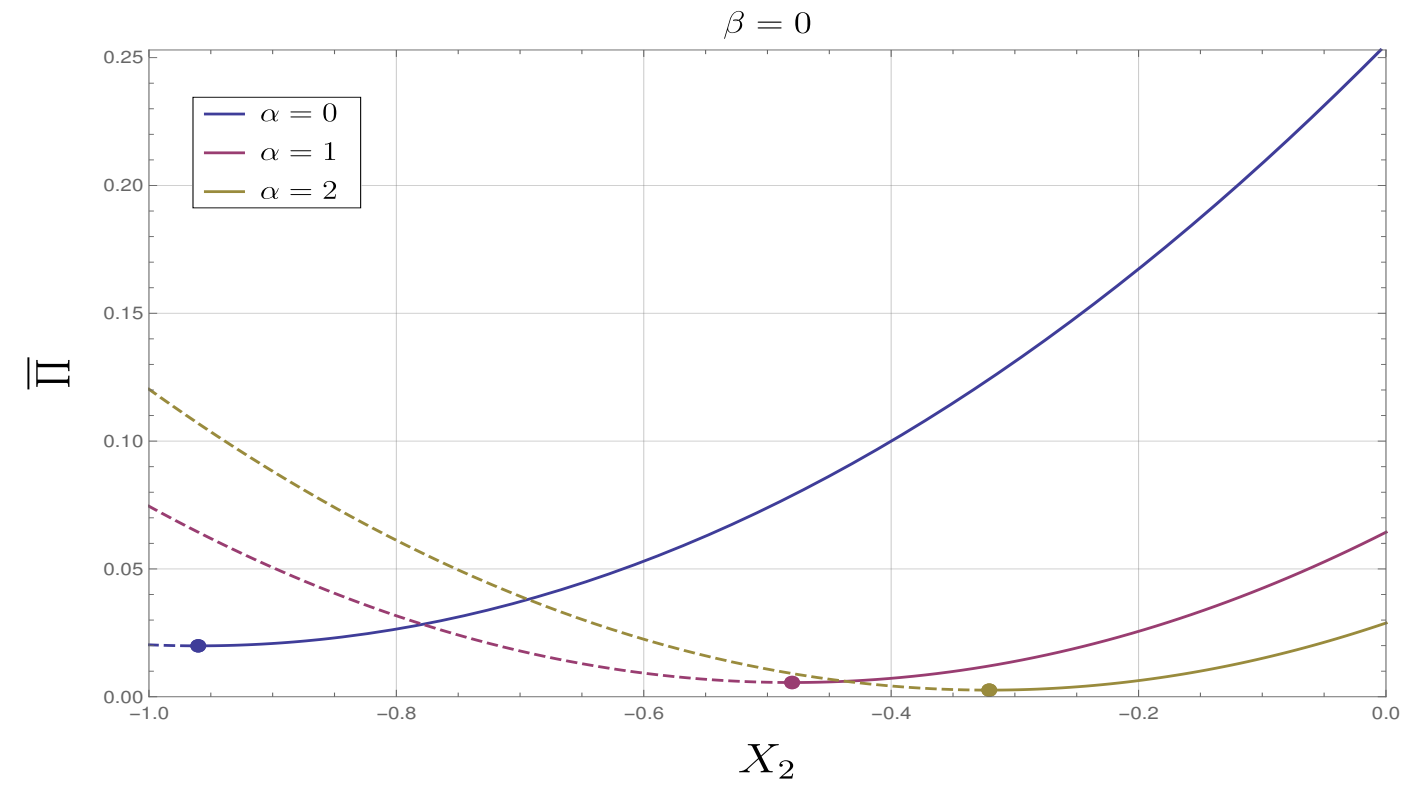

Figura 4.8: Produção de entropia $\bar{\Pi}$ em função da força $X_{2}$ para um conjunto de valores de $\alpha$, fixando $\beta=0, \tau=1, X_{1}=1$ e $\Gamma_{1}=\Gamma_{2}=2$. O ponto representa o valor mínimo da produção de entropia e a linha contínua o intervalo para $X_{2}$ onde o sistema opera como máquina.

Uma vez tendo a expressão (4.2.57) para o caso $\beta=0$ e $\alpha \in\{0,1,2\}$, podemos encontrar uma das grandezas centrais deste trabalho: a eficiência em máxima potência $\eta_{m P}$. A seguir, temos as curvas que representam a eficiência em máxima potência para diferentes valores de $\alpha$, fixando $\beta=0$. 


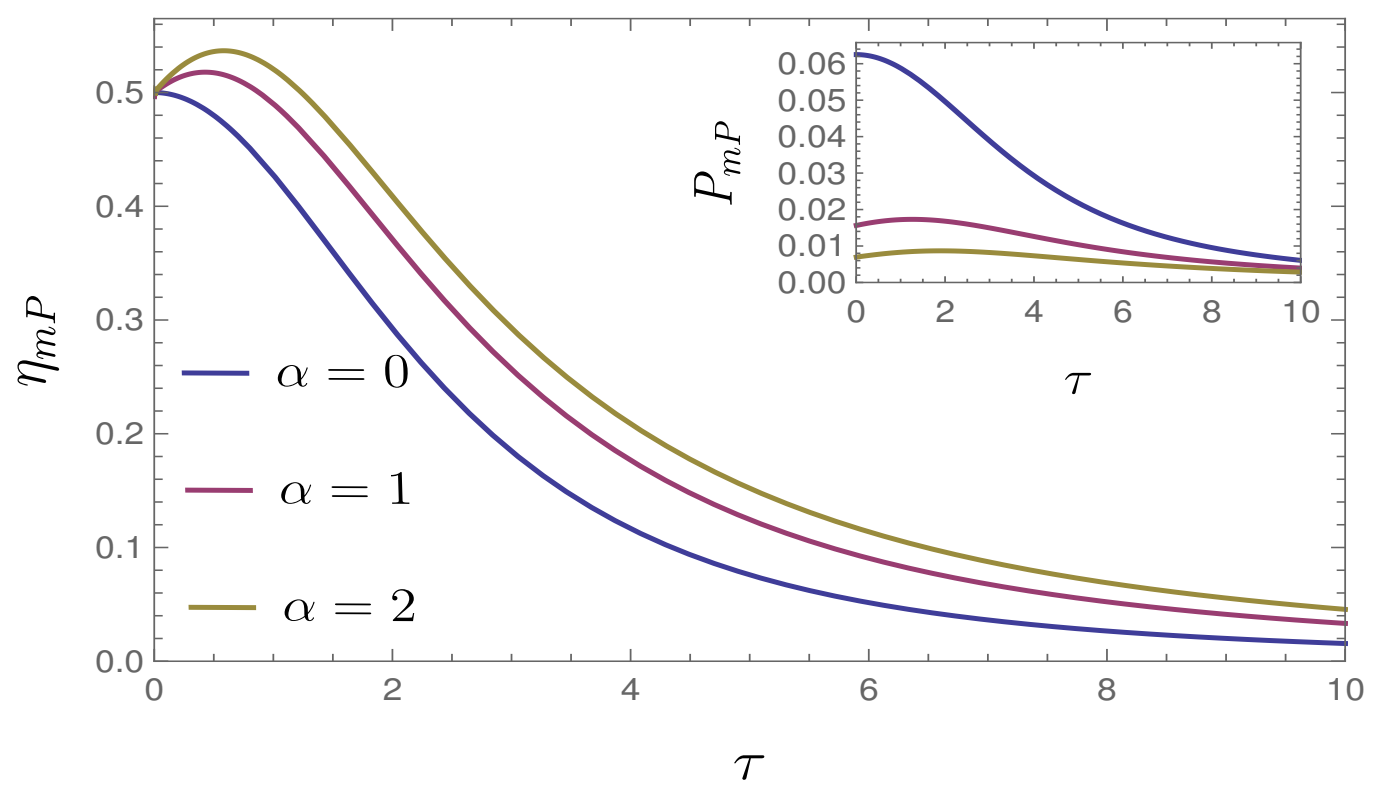

Figura 4.9: Curvas para a eficiência em máxima potência $\eta_{m P}$ em função de $\tau$ para $\beta=0 \mathrm{e}$ alguns valores de $\alpha$. Também é exibido $P_{m P}$ em função de $\tau$ para os mesmos valores de $\alpha$ e $\beta$.

Notamos que para todos os valores de $\alpha$, a eficiência em máxima potência no regime de ciclos rápidos, $\tau<<1, \eta_{m P} \rightarrow 1 / 2$. Podemos notar que, ao aumentar o valor de $\alpha$, diminuimos a potência máxima, mas aumentanmos a eficiência nesse regime.

Considerando os resultados aqui exibidos, podemos escolher qual o melhor regime a se adotar para uma máquina ótima. Em caso de uma máquina mais potente, escolhe-se o caso de forças constantes, enquanto que a máquina mais eficiente deve ser o caso onde $\alpha=2$ e $\beta=0$. Adotaremos os mesmos métodos, agora tomando $\beta=1$. A seguir, exibimos, respectivamente, as curvas para eficiência e para a potência para um conjunto de valores de $\alpha \in\{0,1,2\}$ 

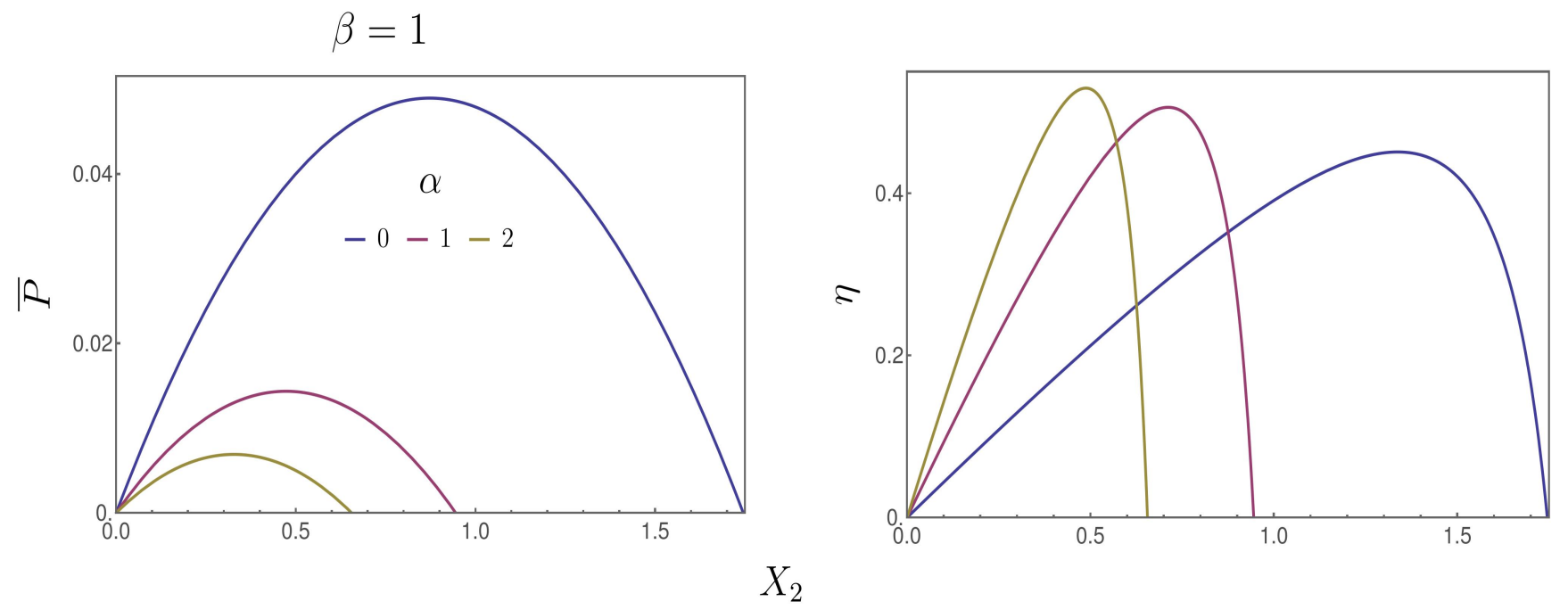

Figura 4.10: Eficiência $\eta$ e potência $\bar{P}$ em função da força $X_{2}$ para um conjunto de valores de $\alpha$, fixando $\beta=1, X_{1}=1, \tau=1$ e $\Gamma_{1}=\Gamma_{2}=2$.

É interessante notar que agora, o intervalo onde a potência útil é gerado ocorre para valores postivos de $X_{2}$. Esse resultado sugere que a troca $\beta \rightarrow \beta+1$ resulta na mudança de sinal da força externa onde a potência externa pode ser gerada. Pelos resultados presentes aqui (incluindo $\beta=2$ ), podemos supor que o sinal de $X_{2}$ será negativo em relação a $X_{1}$ se $\beta$ for par e terá o sinal negativo no caso ímpar. Nessa mudança de sinal, percebemos um aumento no range de $X_{2}$, porém, perde-se em eficiência e potência.

A seguir, exibimos as curvas para a produção de entropia média para o caso $\beta=1$. Notamos que considerar $X_{2}$ operante emtre o mínimo de entropia até o $X_{2}$ até o zero ainda é uma boa estimativa, principalmente ao considerar $\alpha=\beta=1$. 


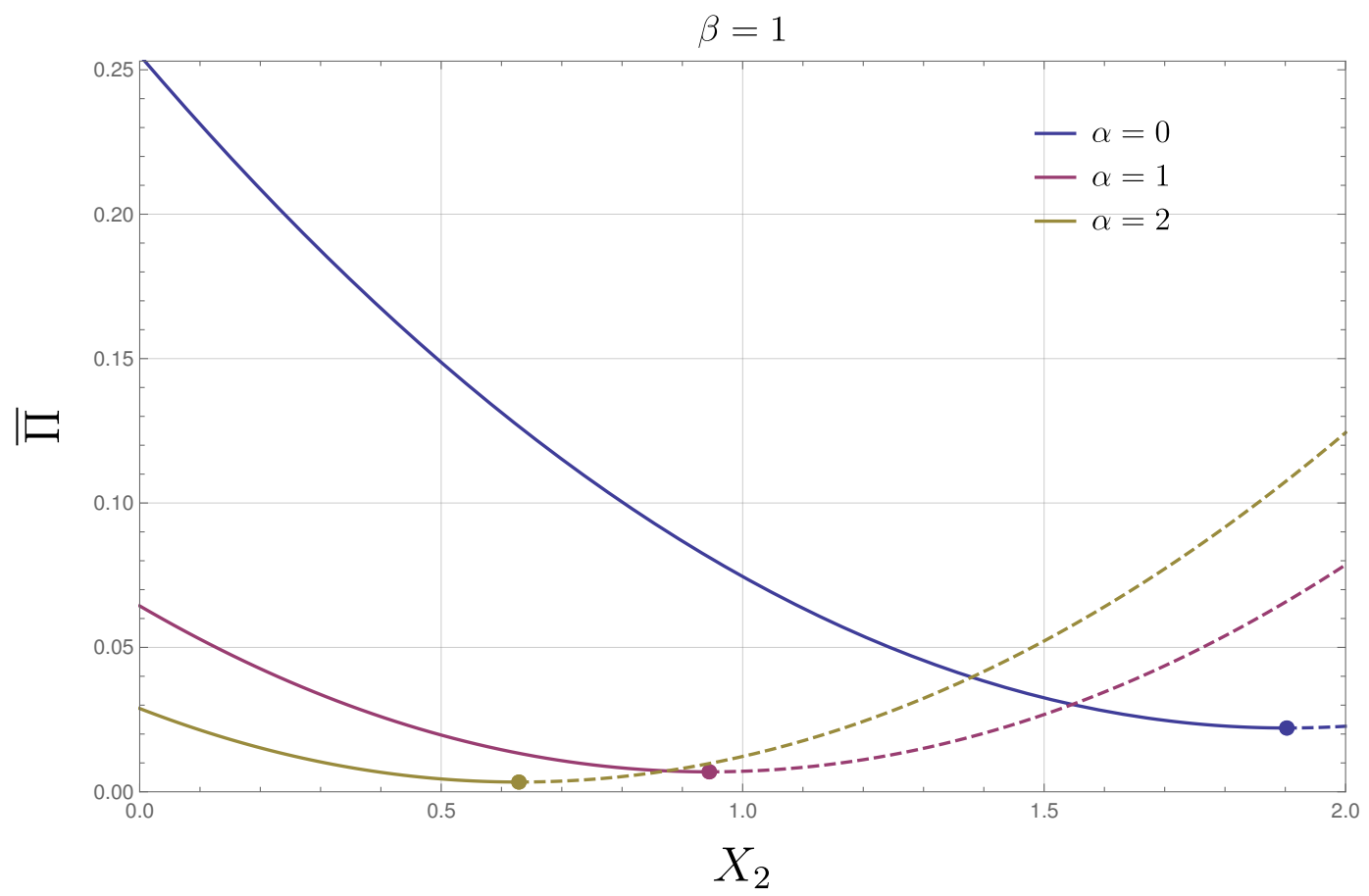

Figura 4.11: Produção de entropia $\bar{\Pi}$ em função da força $X_{2}$ para um conjunto de valores de $\alpha$, fixando $\beta=0, \tau=1, X_{1}=1$ e $\Gamma_{1}=\Gamma_{2}=2$. O ponto representa o valor mínimo da produção de entropia e a linha contínua o intervalo para $X_{2}$ onde o sistema opera como máquina.

Agora, estudaremos a eficiência em máxima potência para o caso $\beta=1$. Mais uma vez, o regime de ciclo rápidos apresenta que $\eta_{m P} \rightarrow 1 / 2$. Para valores maiores de $\tau$, a eficiência para o maior valor de $\alpha$ selecionado é maior, mostrando o mesmo padrão para $\beta=0$. Para ciclos longos, $\tau>>1$, o valor da eficiência em máxima potência também independe de $\alpha \mathrm{e}$ $\beta$. Seus valores são relevantes para ciclos intermediários. 


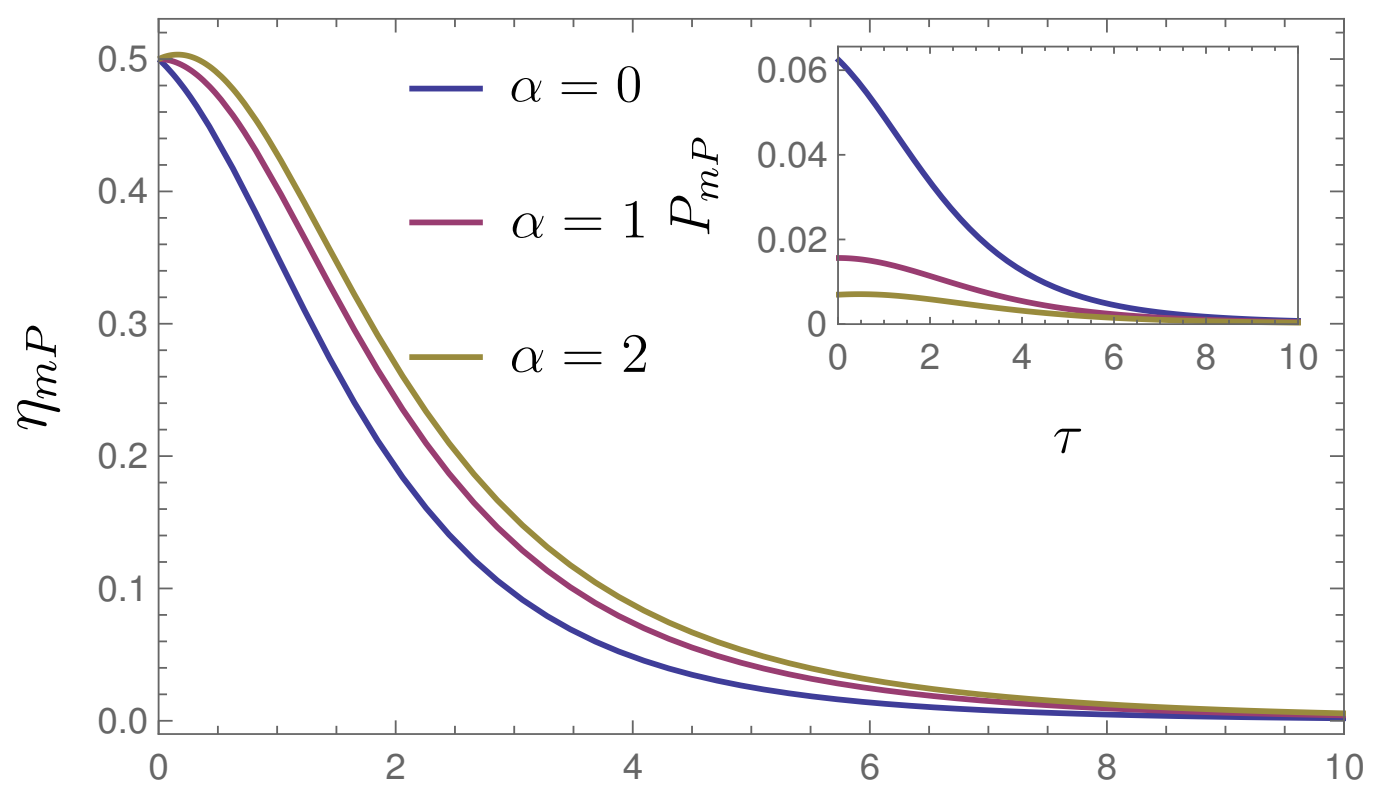

$\tau$

Figura 4.12: Curvas para a eficiência em máxima potência $\eta_{m P}$ em função de $\tau$ para $\beta=1$ e alguns valores de $\alpha$. Também é exibido $P_{m P}$ em função de $\tau$ para os mesmos valores de $\alpha$ e $\beta$.

Finalmente, consideramos $\beta=2$. Mais uma vez, a transformação $\beta \rightarrow \beta+1$ exibe uma mudança de sinal no range de $X_{2}$. Também notamos um aumento considerável no intervalo de $X_{2}$ onde potência útil é gerada em comparação a $\beta=0$. Podemos notar, porém, uma diminuição da potência e da eficiência em relação ao mesmo regime. Agora, a maior eficiência para $\beta=2(\alpha=2)$, é abaixo do $50 \%$. A menor eficiência $(\alpha=0)$ está em torno dos $40 \%$ em seu valor mais alto. Com os resultados preliminares apresentados aqui, podemos concluir que, para os valores estudados de $\alpha$ e $\beta$, conseguimos aumentar a eficiência aumentando $\alpha$ e aumentar a potência diminuindo $\beta$. Notamos na figura (4.14) que considerar o intervalo de valores de $X_{2}$ onde a produção de entropia é mínima até seu ponto nulo ainda continua sendo uma boa estimativa, apesar das assimetrias já discutidas para o caso $\alpha \neq \beta$. 

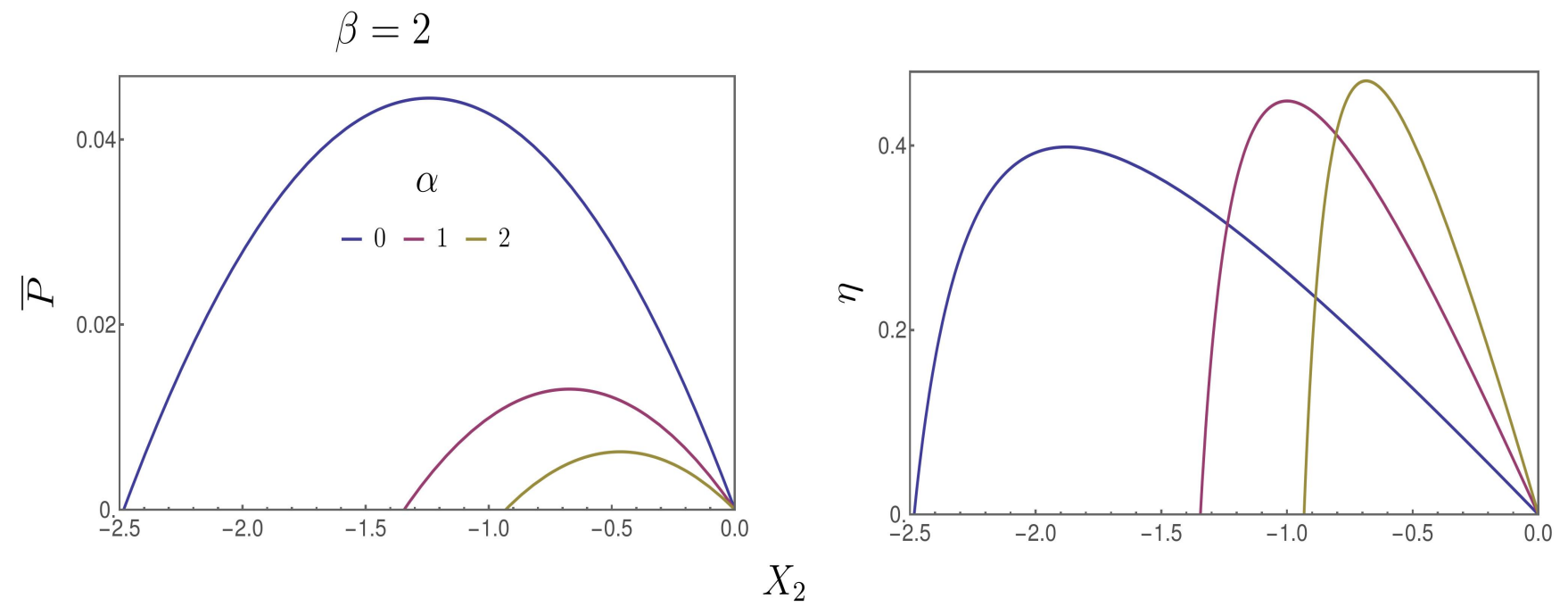

Figura 4.13: Eficiência $\eta$ em função da força $X_{2}$ para três valores de $\alpha$, fixando $\beta=1$, $X_{1}=1, \tau=1$.

$\beta=2$

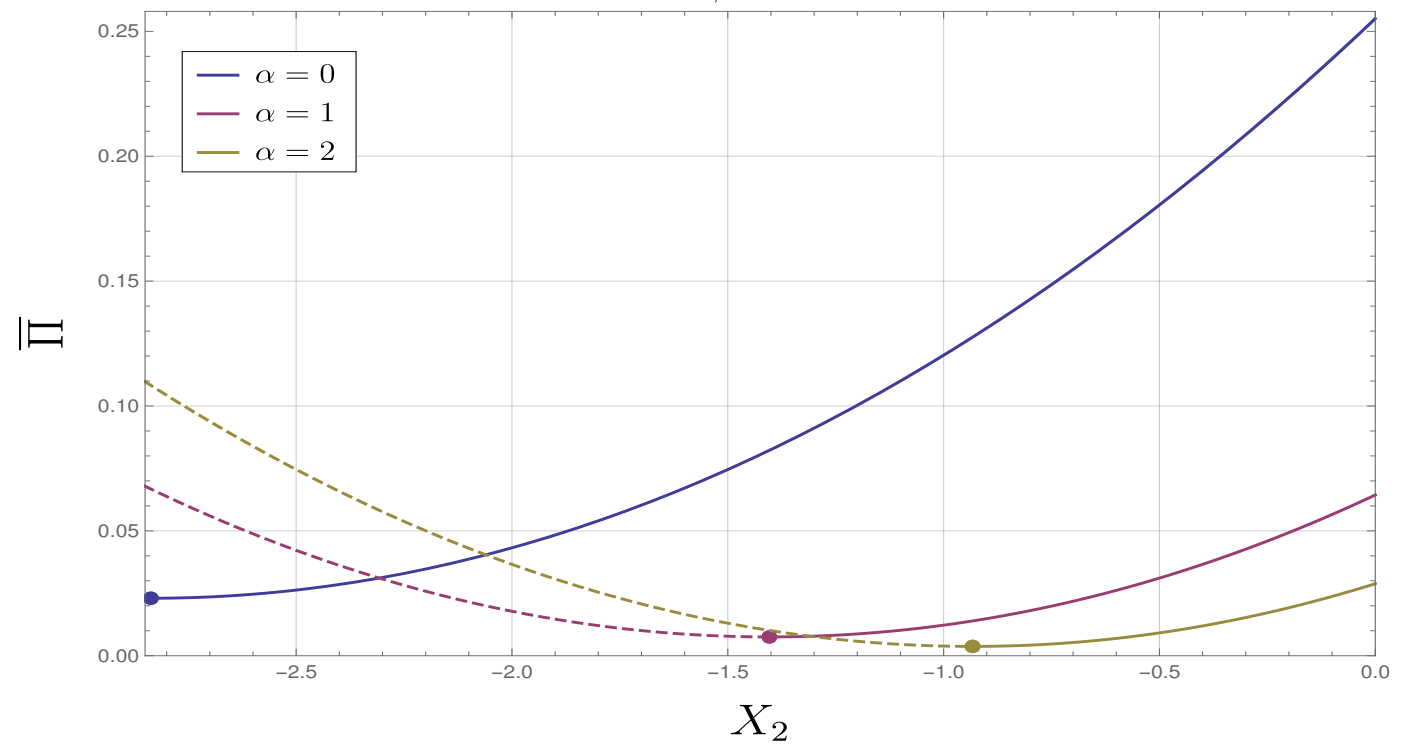

Figura 4.14: Produção de entropia $\bar{\Pi}$ em função da força $X_{2}$ para um conjunto de valores de $\alpha$, fixando $\beta=0, \tau=1, X_{1}=1$ e $\Gamma_{1}=\Gamma_{2}=2$. O ponto representa o valor mínimo da produção de entropia e a linha contínua o intervalo para $X_{2}$ onde o sistema opera como máquina. 


\section{CAPÍTULO 4. MODELO COLISIONAL PARA UMA PARTÍCULA BROWNIANA}

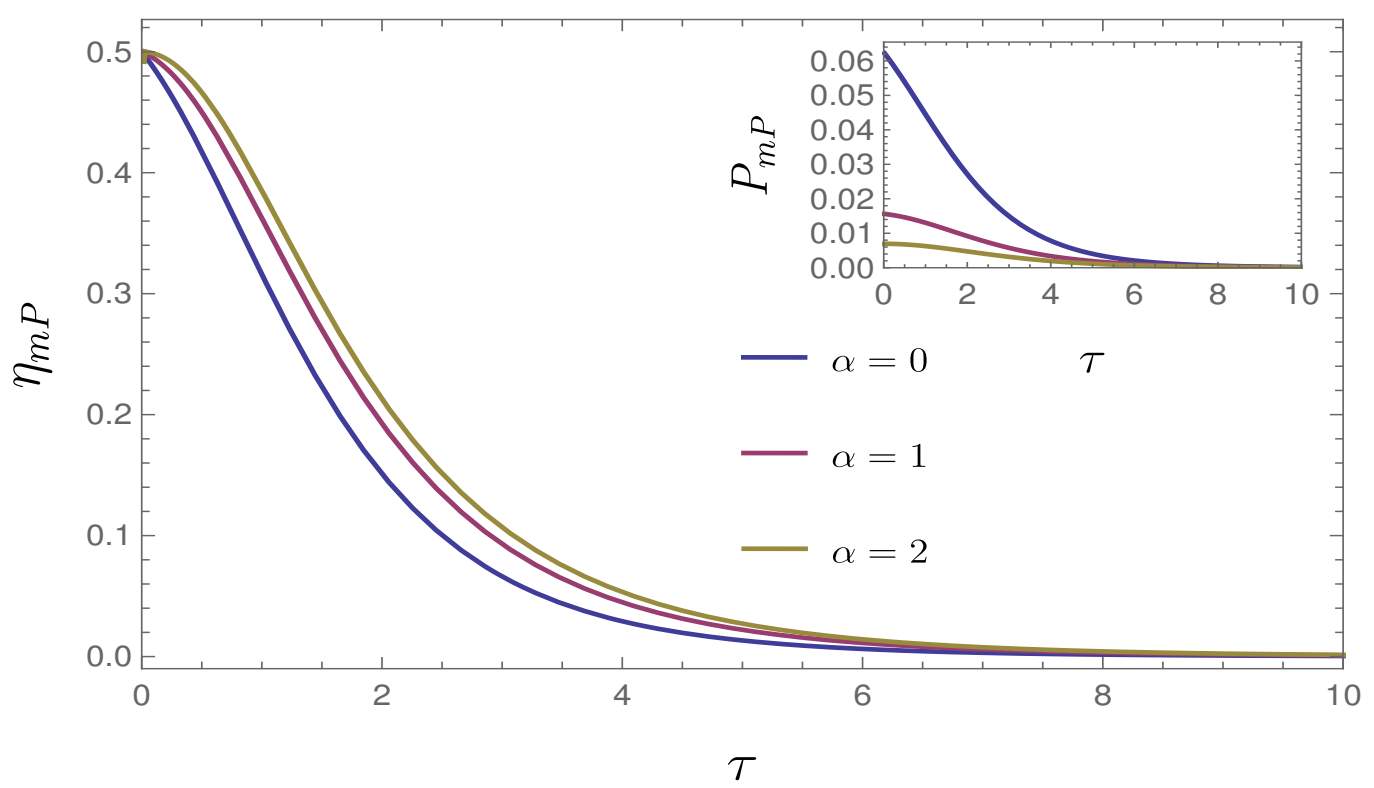

Figura 4.15: Curvas para a eficiência em máxima potência $\eta_{m P}$ em função de $\tau$ para $\beta=2$ e alguns valores de $\alpha$. Também é exibido $P_{m P}$ em função de $\tau$ para os mesmos valores de $\alpha$ e $\beta$.

Outro resultado importante é estudar a eficiência em potência máxima, como exibido na figura (4.15). neste regime, continuamos a enxergar o mesmo padrão para os valores anteriores de $\beta$. A eficiência em seus regimes assintóticos continua independente dos valores de $\alpha$ e $\beta$ e valores mais altos de $\alpha$ continuam oferencendo a melhor eficiência (e menor potência máxima).

Em suma, estudamos a influência de uma força externa com determinadas leis de potências tem em relação à eficiência e à potência. Também calculamos para um caso geral, os fluxos médios de trabalho, a eficiência e a produção de entropia, encontrando sua forma bilinear e os coeficiencentes de Onsager em função dos drives. Esses resultados preliminares sugerem que o aumento de valores de $\beta$ resulta em uma diminuição da eficiência e da potência. Em contrapartida, o aumento de $\alpha$ gera um aumento da primeira. Os pares $(\alpha, \beta)$ nas condições estudadas apresentam um valor máximo de eficiência para $(\alpha=2, \beta=0)$ (respectivamente os maiores e os menores valores de $\alpha$ e $\beta$ ). Em se tratando da potência, a melhor escolha ainda é o caso mais simples $(\alpha=\beta=0)$ mas evidenciamos que é possível aumentar a sua eficiência aumentando valores de $\alpha$. Pretendemos avançar neste estudo 
derivando expresões gerais em termos de $\alpha$ e $\beta$, bem como a influência da diferença entre temperaturas nas etapas 1 e 2.

\subsubsection{Forças Senoidais}

Consideraremos agora uma força externa que é descrita por meio de funções senos, como descrito na expressão (4.2.59) abaixo. No segundo reservatório, ocorre uma mudança de fase $\phi$ no ângulo que depende do tempo de acordo com a regra descrita a seguir. Mesmo no caso onde $\phi=0$, ainda há uma perturbação suficiente para gerar trabalho útil, devido a diferença de forças $X_{1}$ e $X_{2}$. Neste estudo, faremos um estudo geral e numérico, sem se preocupar com a obtenção de uma expressão geral. Os resultados aqui são preliminares e servirão de base para pesquisas futuras.

$$
f(t)=\left\{\begin{array}{l}
f_{1}(t)=X_{1} \sin \left(\frac{2 t}{\tau}\right), t \in[0, \tau / 2] \\
f_{2}(t)=X_{2} \sin \left(\frac{2 t}{\tau}-\phi\right), t \in[\tau / 2, \tau] .
\end{array}\right.
$$

Usando a definição geral de trabalho médio para cada (4.2.5) e (4.2.6) para as forças descritas por (4.2.59), podemos encontrar:

$$
\begin{aligned}
& \overline{\dot{W}}_{1}=-\frac{1}{\tau\left(e^{\gamma \tau}-1\right)}\left[\left(e^{\gamma \tau}-1\right) X_{1}^{2} \int_{0}^{\tau / 2} e^{-\gamma t} \sin (2 t / \tau)\left(\int_{0}^{t} e^{\gamma t^{\prime}} \sin \left(2 t^{\prime} / \tau\right) d t^{\prime}\right) d t+\right. \\
& \quad X_{1}^{2} \int_{0}^{\tau / 2} e^{\gamma t^{\prime}} \sin \left(2 t^{\prime} / \tau\right) d t^{\prime} \int_{0}^{\tau / 2} e^{-\gamma t} \sin (2 t / \tau) d t+e^{\gamma \tau / 2} X_{1} X_{2} \int_{\tau / 2}^{\tau} e^{\gamma\left(t^{\prime}-\tau / 2\right)} \sin \left(2 t^{\prime} / \tau-\phi\right) d t^{\prime} \times \\
& \left.\quad \times \int_{0}^{\tau / 2} e^{-\gamma t} \sin (2 t / \tau) d t\right] \\
& \overline{\dot{W}}_{2}=-\frac{1}{\tau\left(e^{\gamma \tau}-1\right)}\left\{( e ^ { \gamma \tau } - 1 ) \left[X_{2}^{2} \int_{\tau / 2}^{\tau} e^{-\gamma(t-\tau / 2)} \sin (2 t / \tau-\phi)\left(\int_{\tau / 2}^{\tau} e^{\gamma\left(t^{\prime}-\tau / 2\right)} \sin \left(2 t^{\prime} / \tau-\phi\right) d t^{\prime}\right) d t+\right.\right. \\
& \left.e^{-\gamma \tau / 2} X_{1} X_{2} \int_{0}^{\tau / 2} e^{\gamma t^{\prime}} \sin \left(2 t^{\prime} / \tau-\phi\right) d t^{\prime} \int_{\tau / 2}^{\tau} e^{-\gamma(t-\tau / 2)} \sin (2 t / \tau-\phi) d t\right]+X_{2}\left[X_{1} \int_{0}^{\tau / 2} e^{\gamma\left(t^{\prime}-\gamma \tau / 2\right)} \sin \left(2 t^{\prime} / \tau\right) d t^{\prime}\right. \\
& \left.\left.+X_{2} \int_{\tau / 2}^{\tau} e^{\gamma\left(t^{\prime}-\tau / 2\right)} \sin \left(2 t^{\prime} / \tau-\phi\right) d t^{\prime} \int_{\tau / 2}^{\tau} e^{-\gamma(t-\tau / 2)} \sin (2 t / \tau-\phi) d t\right] \int_{\tau / 2}^{\tau} e^{-\gamma(t-\tau / 2)} \sin (2 t / \tau-\phi) d t\right\}
\end{aligned}
$$

As integrações acima podem ser realizadas usando uma série de métodos de integração de funções complexas. Na atual conjuntura, estamos interessado em resultados numéricos para 
alguns conjuntos de ângulos. Mais uma vez lidamos com uma máquina de reservatórios com temperaturas iguais e portanto, temos um sistema "work-work converter"como nos casos anteriores, com a eficiência dada pela expresssão (4.2.39). A potência é dada pela expressão de $\bar{W}_{2}$. Vamos inicialmente considerar os seguintes ângulos: $\{0, \pi / 4,3 \pi / 10,1\}$. A seguir, apresentamos as curvas da potência em função de $X_{2}$, fixando $X_{1}=1$.
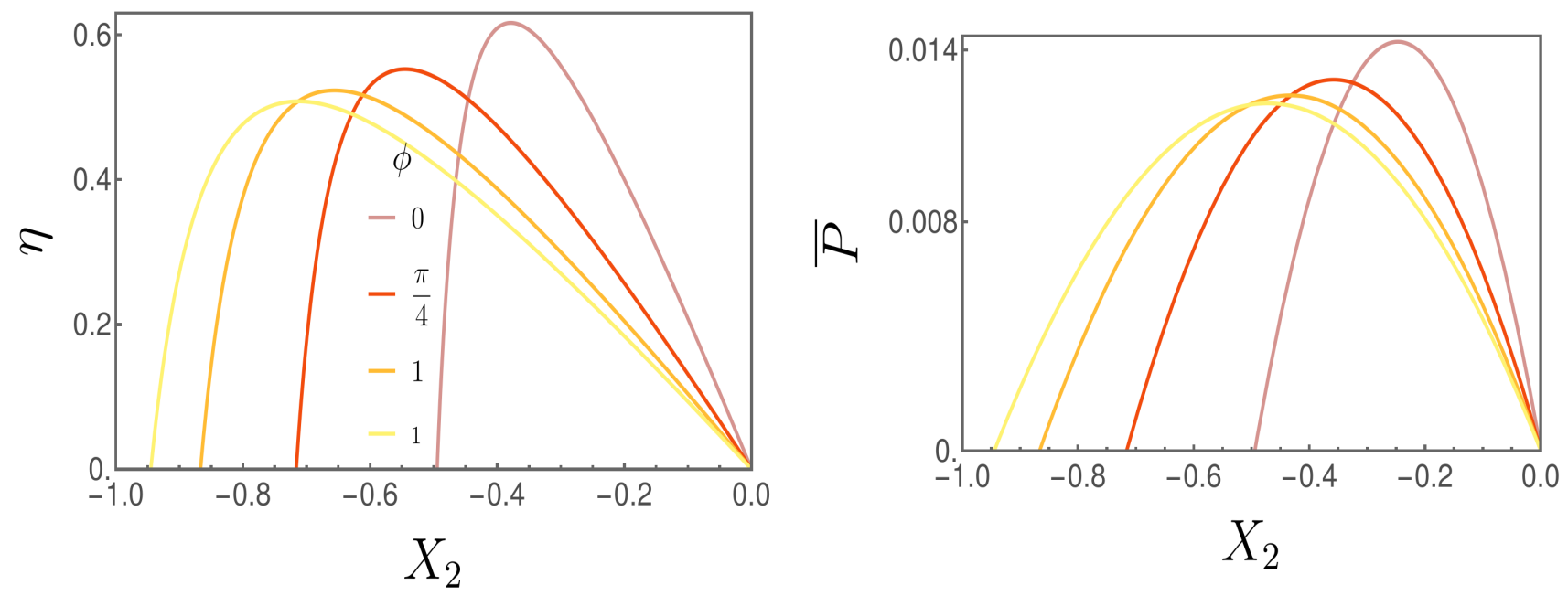

Figura 4.16: Eficiência $\eta$ e potência média $P=\bar{W}_{2}$ em função da força $X_{2}$ para um conjunto de valores de $p h i$, fixando $\tau=1, X_{1}=1$ e $\Gamma_{1}=\Gamma_{2}=2$.

Podemos notar que, para o intervalo de difrenças de fase escolhido, a potência diminui. Mesmo para o seu valor mais alto $(\phi=0)$, a potência ainda é menor que alguns valores considerados para os casos anteriores. Já para a eficiência, de acordo com a expressão (4.2.39), os gráficos são apresentados na Fig.(4.16). Numericamente, a eficiência é comparável a casos de maior eficiência para o caso polinomial, como as descritas na Fig. (4.7). A eficiência para $\phi=0$ é comparável à eficiência para $\alpha=2$ e $\beta=0$, com a diferença que o intervalo da força $X_{2}$ por onde o sistema opera como máquina é maior para este caso em comparação com o caso polinomial de maior eficiência. Isso se reforça ao comparar o ponto de menor entropia para o caso trigonométrico com a curva (4.7) para $\alpha$ e $\beta$ supracitados.

Como nos casos anteriores, estimar o valor de $X_{2}$ para qual o mínimo da produção de entropia é alcançada ainda é vantajoso para estimar a região onde o sistema opera como máquina. Podemos notar na figura (4.17) que a região de linhas contínuas, partindo do valor 
mínimo $X_{2 m E}$ até $X_{2}=0$, coincide com a região de eficiência e potência positivas.

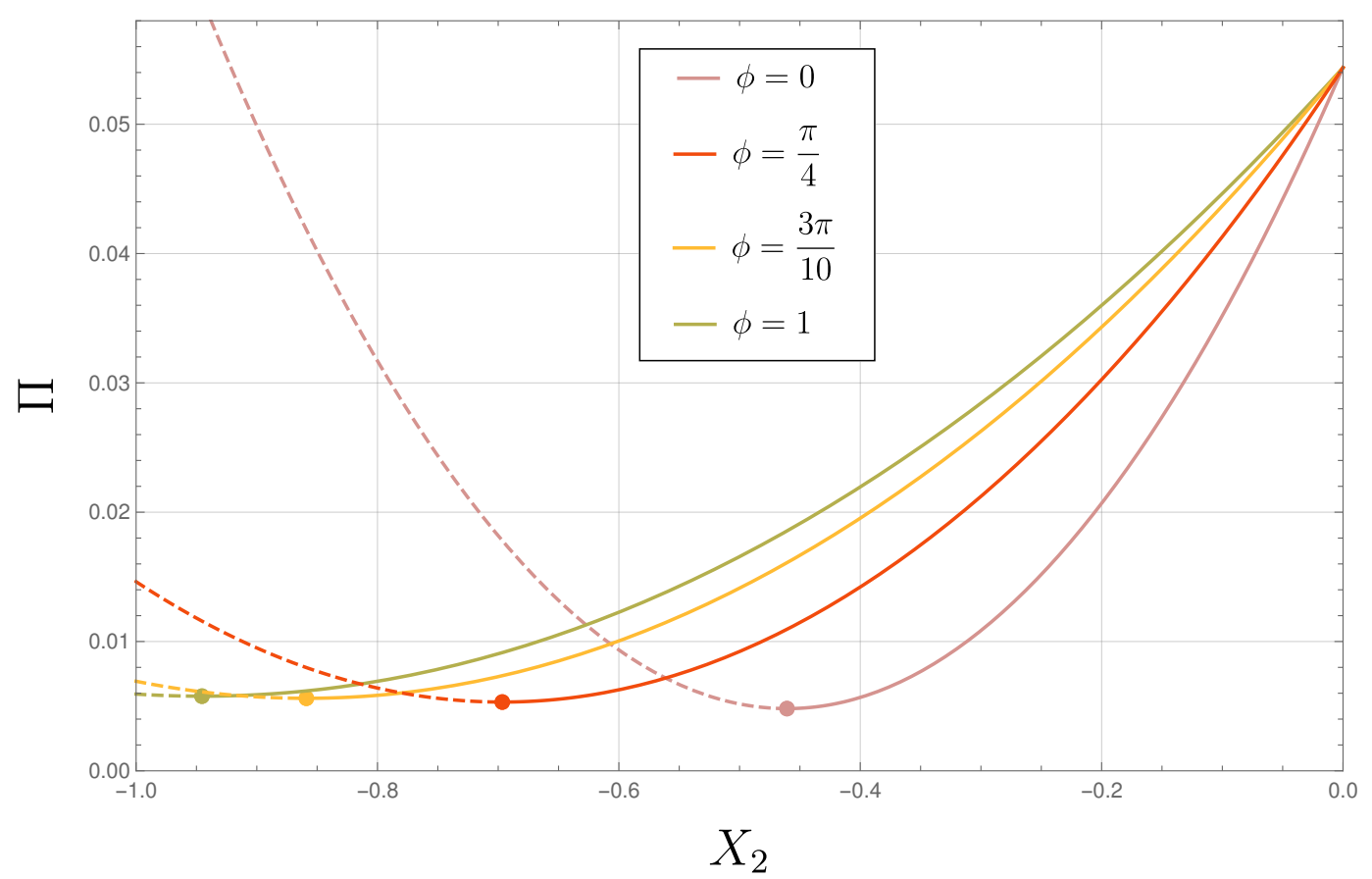

Figura 4.17: Produção de entropia $\bar{\Pi}$ em função da força $X_{2}$ para um conjunto de valores de $\phi$, fixando $\tau=1, X_{1}=1$ e $\Gamma_{1}=\Gamma_{2}=2$. O ponto representa o valor mínimo da produção de entropia e a linha contínua o intervalo para $X_{2}$ onde o sistema opera como máquina.

Outra característica notável deste modelo em relação ao intervalo de $X_{2}$ ocorre ao considerar a eficiência e a potência para os ângulos somados com um fator de fase $\pi$, isto é, considerar $\{0, \pi / 4,1\} \rightarrow\{\pi, 5 \pi / 4,1+\pi\}$. Ao somar a diferença de fase com $\pi$, invertemos o sinal do intervalo de força onde o sistema opera como máquina. Diferente do que ocorre para o caso polinomial, onde conseguimos o mesmo efeito na transição $\beta \rightarrow \beta+1$ mas a potência e a eficiência diminuem, no caso senoidal a potência e a eficiência apresentam o mesmo valor, como evidenciamos nas figuras (4.7). 


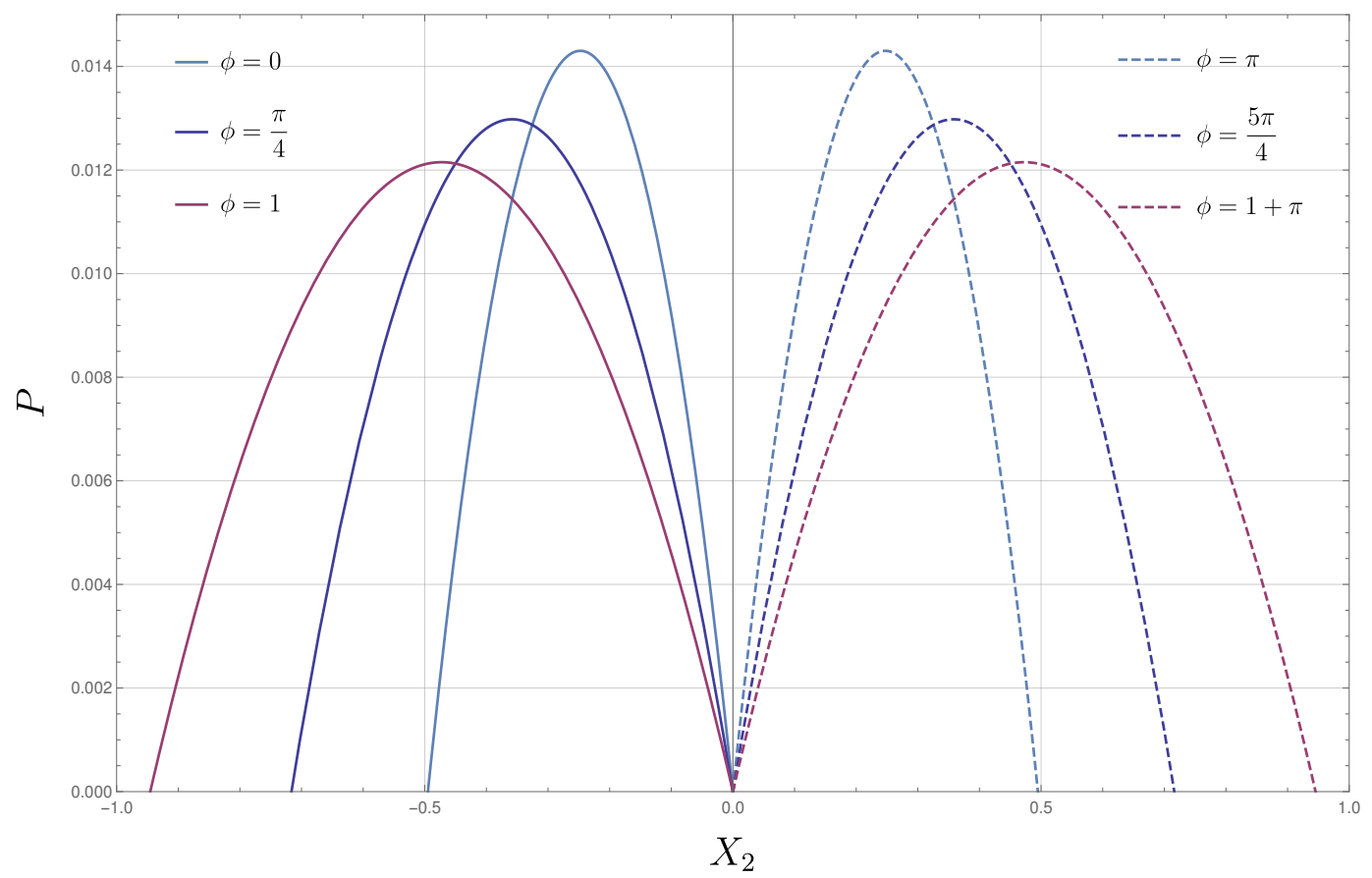

Figura 4.18: Potência $P=\bar{W}_{2}$ em função da força $X_{2}$ para um conjunto de valores de $\phi$, representados no lado esquerdo por linhas contínuas e para um conjunto de valores de $\phi+1$ representados no lado direito por linhas tracejadas. fixamos $\tau=1, X_{1}=1$ e $\Gamma_{1}=\Gamma_{2}=2$. 


\section{CAPÍTULO 4. MODELO COLISIONAL PARA UMA PARTÍCULA BROWNIANA}

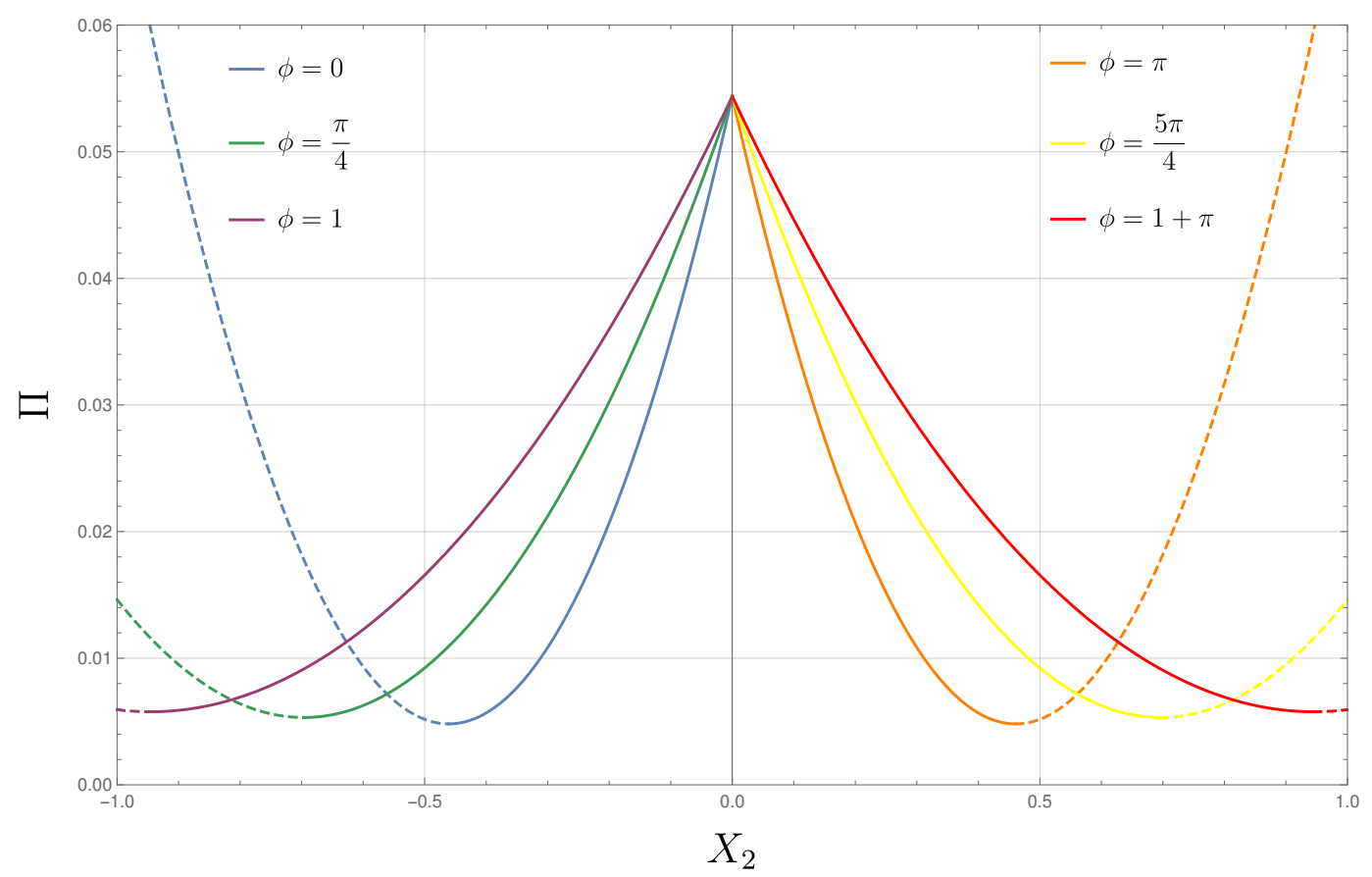

Figura 4.19: Produção de entropia $\bar{\Pi}$ em função da força $X_{2}$ para um conjunto de valores de $\phi$, representados no lado esquerdo e para um conjunto de valores de $\phi+1$, representados no lado direito. As linhas contínuas representam os intervalos de $X_{2}$ onde o sistema opera como máquina. Fixamos $\tau=1, X_{1}=1$ e $\Gamma_{1}=\Gamma_{2}=2$.

Para esse caso, também estudamos a eficiência em potência máxima. Como evidenciado pela figura (4.20), não há diferenças significativas em relação aos casos anteriores, coma a difrença do que é observado para o ângulo $\phi=\pi / 4$, onde, no regime assintótico, $\eta_{m P}<1 / 2$. Isso torna a máquina mais desvantajosa em relação aos outros casos estudados aqui. É interessante notar que a potência máxima $P_{m P}$ para o caso assintótico é o mesmo para $\phi=1$ e $\phi=0$. Esse valor é aproximadamente a potência máxima para os casos menos eficiêntes estudados anteriormente. Concluímos disso que o caso das forças constantes, além de mais simples, são mais potentes. 


\section{CAPÍTULO 4. MODELO COLISIONAL PARA UMA PARTÍCULA BROWNIANA}

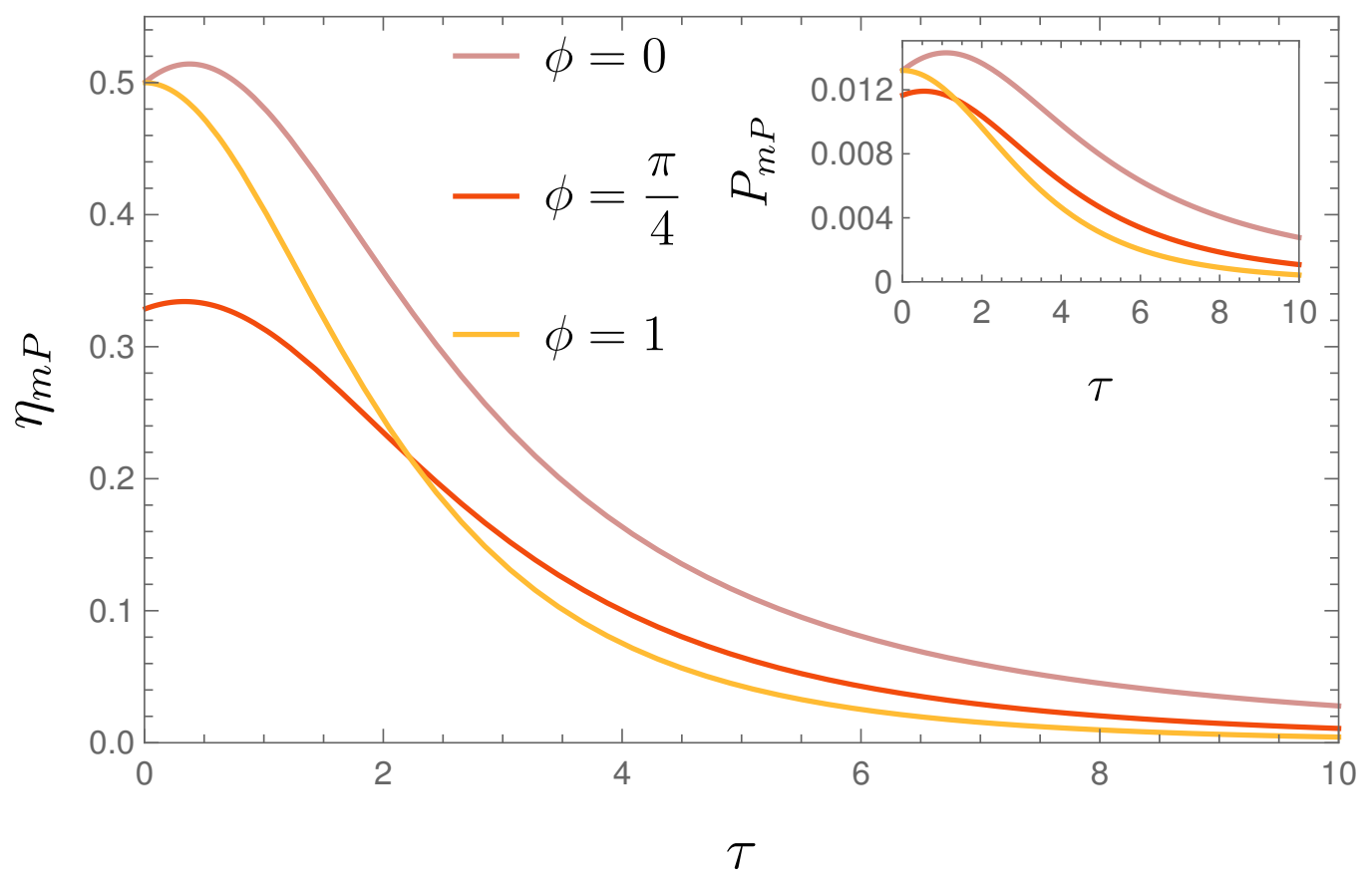

Figura 4.20: $\beta=2$

Em suma, estudamos as principais grandezas termodinâmicas, bem como a eficiência, para diferentes dependências temporais dos drive. Também explicitamos, em termos das forças externas, a forma bilinear da produção de entropia e os coeficientes de Onsager. Também conseguimos estudar a eficiência e máxima potência, resultado central deste trabalho. Mostramos que no regime de ciclos rápidos $(\tau<<1)$, a eficiência converge para o valor $1 / 2$ independente da configuração do drive, com exceção de alguns ângulos para o caso senoidal. Enfatizamos que os resultados presentes aqui são preliminares e portanto, existem muitas questões não respondidas aqui que pretendemos esclarecer em investigações futuras. Entre elas, citamos a necessidade de uma expressão geral do trabalho e da eficiência para ambos os casos, bem como expressarmos a otimização da máquina em termos dos coeficientes de Onsager e a influência da diferença de temperaturas nas etapas 1 e 2 . 


\section{Capítulo 5}

\section{Conclusão}

Neste trabalho, estudamos modelos colisionais para um sistema composto por um quantumdot e por uma partícula browniana, ambos interagindo termicamente e quimicamente (no caso do quantum dot) com um reservatório. Com base na termodinâmica estocástica, conseguimos calcular grandezas importantes como o fluxo de trabalho, calor e eficiência em máxima potência. Para o caso da produção de entropia média, encontramos a sua forma bilinear e os coeficientes de Onsager explicitamente em função de características do drives para o caso browniano.

Para o sistema de quantum-dot, analisamos a influência no tempo de interação do sistema com o reservatório com métodos matematicamente simples de maximização. Nossos resultados mostram que a assimetria no tempo de interação pode aumentar a potência da máquina em até $25 \%$ em comparação com o valor obtido para o regime simétrico. Como resultado adicional, mostramos que a eficiência em máxima potência supera o rendimento CurzonAhlborn para as mesmas temperaturas para alguns regimes. Um ponto muito importante dos nossos resultados é que a maximização em relação ao tempo é muito mais fácil de se implementar do que em relação ao design ou a outros métodos de maximização. Além disso, essa análise abre um precedente para discussões sobre escalas de tempo e a sua importância na performance de uma máquina térmica.

No caso browniano, analisamos a influência de diversas dependências temporais de drives 
na eficiência e na potência de uma máquina térmica operando sobre elas. Em particular, estudamos como essas novas variáveis influenciam na produção de entropia. Conseguimos encontrar expressões gerais para a potência média extraída em função de uma força externa genérica. Os estudos dessa parte são preliminares, mas serviram para evidenciar alguns resultados importantes. Em relação ao caso polinomial, podemos aumentar a eficiência ou a potência manipulando os valores dos expoentes. Em se tratando de maior potência, o caso constante (expoentes nulos) ainda é mais vantajosa. Para casos assintóticos, a eficiência em máxima potência independe dos valores do expoente, sendo que seu valor para ciclos rápidos tende para $1 / 2$ pra todos os casos estudados. Para o caso senoidal, a eficiência é a potência são consideravelmente menores em comparação aos casos anteriores, porém apresenta resultados que merecem melhor investigação, principalmente em regimes de ciclos rápidos da eficiência em máxima potência, bem como uma exploração mais detalhada do papel da defasagem entre as forças. 


\section{Apêndice A}

\section{Artigo Publicado}

Anexamos a seguir o nosso artigo publicado na revista Physical Review Research correspondente aos resultados do Capítulo 3 desta dissertação. Foi um trabalho feito em colaboração com, além do meu orientador, o Pedro Harunari (aluno de doutorado do IF-USP) e com o Prof. Alexandre Rosas (UFPB). Pretendemos desenvolver melhor os resultados do Capítulo 4 e confeccionar um artigo para ser publicado em breve. 


\author{
Maximal power for heat engines: Role of asymmetric interaction times \\ Pedro E. Harunari, ${ }^{1, *}$ Fernando S. Filho $\odot,{ }^{1, \dagger}$ Carlos E. Fiore $\odot, 1,+$ and Alexandre Rosas ${ }^{2, \S}$ \\ ${ }^{1}$ Instituto de Física da Universidade de São Paulo, 05508-090 São Paulo, SP, Brazil \\ ${ }^{2}$ Departamento de Física, CCEN, Universidade Federal da Paraíba, Caixa Postal 5008, 58059-900 João Pessoa, Brazil
}

(Received 18 December 2020; revised 2 March 2021; accepted 4 May 2021; published 9 June 2021)

\begin{abstract}
The performance of endoreversible thermal machines operating at finite power constitutes one of the main challenges of nonequilibrium classical and quantum thermodynamics, engineering, and others. We introduce the idea of adjusting the interaction time asymmetry in order to optimize the engine performance. We consider one of the simplest thermal machines, composed of a quantum dot interacting sequentially with two different reservoirs of heat and particles. Distinct optimization protocols are analyzed in the framework of stochastic thermodynamics. Results reveal that asymmetric interaction times play a fundamental role in enhancing the power output and that maximizations can provide an increase of more than $25 \%$ compared with the symmetric case. As an extra advantage, efficiencies at maximum power are slightly greater than the endoreversible CurzonAhlborn efficiency for a broad range of reservoir temperatures.
\end{abstract}

DOI: 10.1103/PhysRevResearch.3.023194

\section{INTRODUCTION}

The efficiency of any heat engine is bounded by Carnot efficiency $\eta_{\mathrm{C}}=1-T_{\mathrm{C}} / T_{\mathrm{H}}$, with $T_{\mathrm{C}}$ and $T_{\mathrm{H}}$ being the cold and hot reservoir temperatures. It constitutes one of the main results of thermodynamics and is one of the distinct formulations of the second law. Such an ideal limit was introduced by Carnot in 1824 [1,2] and consists of a reversible machine composed by two isothermal and two adiabatic quasistatic strokes. Although it is a universal upper bond valid for all engines, irrespective of their designs, compositions, or nature, whether classical $[3,4]$ or quantum $[5,6]$, such an (ideal) limit is impractical, not only due to imperfections in the machine construction, which increases the dissipation, but also because its achievement would demand the machine to operate in a fully reversible way during infinitely large times, implying its operation at a null power (finite work divided by infinite time).

Thus it is usually desirable to build thermal machines to be as efficient as possible operating at finite power outputs. One of the main findings for endoreversible thermal machines is the Curzon and Ahlborn efficiency [7], in which the efficiency at maximum power is given by $\eta_{\mathrm{CA}}=1-\sqrt{T_{\mathrm{C}} / T_{\mathrm{H}}}$. Such a remarkable finding has also been derived in several distinct works (see, e.g., Refs. [8,9]), and despite not possessing the same universal status as the Carnot efficiency, it provides a

\footnotetext{
*pedroharunari@gmail.com

†fernando.francisco.filhof@gmail.com

¥fiore@if.usp.br

§arosas@ fisica.ufpb.br
}

Published by the American Physical Society under the terms of the Creative Commons Attribution 4.0 International license. Further distribution of this work must maintain attribution to the author(s) and the published article's title, journal citation, and DOI. powerful guide as to the operation of nonequilibrium engines under more realistic situations and sheds light on the construction and performance of small-scale engines (nanoscopic devices) working in a maximum power regime from the tools of stochastic thermodynamics [3,4,9-24]. In this context, single-level quantum dots have been proposed as prototype machines, whose simplicity allows detailed investigation of their performances at maximum power [25-27].

Collisional models, e.g., a system interacting sequentially and repeatedly with distinct environments (instead of continuous interaction with all the reservoirs), have been considered as a suitable description of engineered reservoirs [28]. Among the distinct situations for that, we mention the case of quantum systems, in which the reservoir is conveniently represented as a sequential collection of uncorrelated particles [29,30]. Additionally, the collisional approach attempts to provide realistic systems interacting only with small fractions of the environment or even those evolving under the influence of distinct drivings over each member [31,32]. Particularly, many aspects of a stochastic pump in which a single-level quantum dot (QD) is connected sequentially and periodically to different reservoirs have been discussed lately for symmetric interaction times [33-35].

In this paper, we introduce the idea of adjusting the interaction time asymmetry in order to optimize the engine performance. The present approach is rather different from some findings $[10,19,36,37]$ exactly because we explore this adjustment of the interaction time; that is, the interaction time is the focus of our study. Despite the simplicity of the system, its large applicability and richness allow its usage as heat engine, refrigerator, heater, or accelerator, hence highlighting the importance of searching for optimized protocols. As a main finding, under suited situations, asymmetric interaction times play an important role in the enhancement of power output. Also, as an extra advantage, efficiencies become somewhat greater than the endoreversible Curzon-Ahlborn efficiency. 

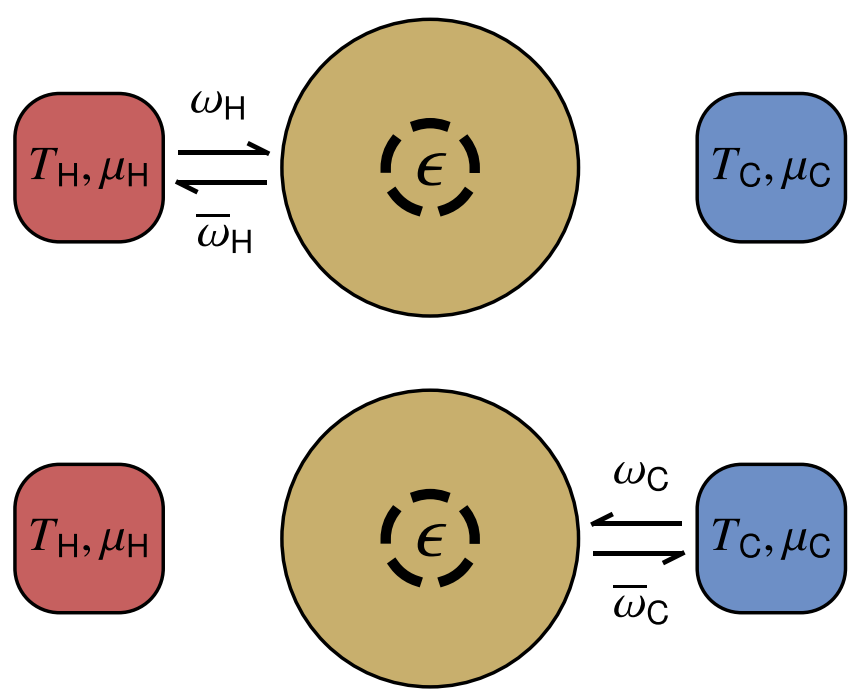

FIG. 1. Sketch of a quantum dot periodically and sequentially placed in contact with a hot (during a time $\tau_{\mathrm{H}}$ ) and a cold (during a time $\tau-\tau_{\mathrm{H}}$ ) reservoir. When in contact with a reservoir, the quantum dot receives a particle with rate $\omega_{i}$ and donates a particle with rate $\bar{\omega}_{i}$ ( $i$ being $\mathrm{H}$ for the hot reservoir and $\mathrm{C}$ for the cold one). When the quantum dot is occupied, its energy increases by $\epsilon$.

This paper is organized as follows: In Sec. II the model is presented, and analytical results are provided. Section III is devoted to the main results concerning the efficiency in different regimes and optimized power outputs. Lastly, in Sec. IV we draw conclusions and provide perspectives.

\section{MODEL AND EXACT SOLUTION}

The model consists of a two-level system sequentially and periodically (with period $\tau$ ) placed in contact with a hot $(\mathrm{H})$ and a cold $(\mathrm{C})$ reservoir during the time intervals $\tau_{\mathrm{H}}$ and $\tau-\tau_{\mathrm{H}}$, respectively, as depicted in Fig. 1. More specifically, the QD interacts with one reservoir during a certain time. Afterwards, one turns off this interaction, and then the QD is placed in contact with the second reservoir. The switching time is assumed to be instantaneous or, at least, much faster than any other relevant time scales. The energy of the QD is null $(\epsilon)$ when it is empty (occupied by one electron). Each interaction can be modeled according to the transition rates $\omega_{i}$ and $\bar{\omega}_{i}$, whether the system receives or delivers a particle, respectively, with $i \in\{\mathrm{H}, \mathrm{C}\}$. Giving that the system placed in contact with a single reservoir evolves to the equilibrium distribution, the connection between transition rates and macroscopic quantities can be performed by assuming that the probability of occupation at equilibrium $p_{i}^{\text {eq }} \equiv \omega_{i} /\left(\omega_{i}+\bar{\omega}_{i}\right)$ obeys the Fermi-Dirac dis- tribution $p_{i}^{\mathrm{eq}}=\left[e^{\left(\epsilon-\mu_{i}\right) / T_{i}}+1\right]^{-1}$, where, for each reservoir, $T_{i}$ is the temperature, $\mu_{i}$ is the chemical potential, and the Boltzmann constant is set to 1 . The assumption above is equivalent to the local detailed balance condition $\omega_{i} / \bar{\omega}_{i}=$ $e^{-\left(\epsilon-\mu_{i}\right) / T_{i}}$, and therefore the temperature of each reservoir is given by

$$
T_{i}=\frac{\mu_{i}-\epsilon}{\ln \omega_{i} / \bar{\omega}_{i}}
$$

As long as the reservoirs are different, the system will evolve to a periodic and time asymmetric nonequilibrium steady state (NESS), in which time reversal means exchanging the order of the reservoirs. An important quantity is the ratio of the transition rates $\omega_{i} / \bar{\omega}_{i}$, which quantifies the reservoir willingness to concede a particle to the QD, equilibrium being reached for $\omega_{\mathrm{H}} / \bar{\omega}_{\mathrm{H}}=\omega_{\mathrm{C}} / \bar{\omega}_{\mathrm{C}}$.

We start the analysis of this system by considering a Markov chain whose discrete time is given by $t \equiv n h$, where $n=0,1,2, \ldots$ and $h$ is the time step. When the system is placed in contact with reservoir $i$, the transition matrix is given by

$$
\mathcal{W}_{i} \equiv\left(\begin{array}{cc}
-\omega_{i} & \bar{\omega}_{i} \\
\omega_{i} & -\bar{\omega}_{i}
\end{array}\right) .
$$

The probability distribution obeys the relation $\vec{P}_{i}(t+n h)=$ $\left(\mathbb{I}+h \mathcal{W}_{i}\right)^{n} \vec{P}_{i}(t)$, where $\mathbb{I}$ is the $2 \times 2$ identity matrix and $\vec{P}_{i}(t) \equiv\left\{1-p_{i}(t), p_{i}(t)\right\}$ is the vector of probabilities of emptiness and occupation. Hence $p_{i}(t+n h)$ can be written as

$$
p_{i}(t+n h)=p_{i}^{\mathrm{eq}}+\left[1-h \bar{\omega}_{i}\left(1+\omega_{i} / \bar{\omega}_{i}\right)\right]^{n}\left(p_{i}(t)-p_{i}^{\mathrm{eq}}\right) .
$$

Since $p_{i}(t)$ is continuous, one has the boundary conditions $p_{\mathrm{H}}\left(\tau_{\mathrm{H}}\right)=p_{\mathrm{C}}\left(\tau_{\mathrm{H}}\right)$. Furthermore, the periodicity of the system ensures that it returns to the initial state after a complete period for long enough times such that $p_{\mathrm{H}}(0)=p_{\mathrm{C}}(\tau)$. The occupation probability can be exactly obtained considering these boundary conditions and solving Eq. (3). Hereafter we consider the NESS regime, for which such boundary conditions are valid.

The NESS particle flux at a given time interval $h$ is given by $J_{\mathrm{i}}(n h) \equiv\left[p_{\mathrm{i}}((n+1) h)-p_{\mathrm{i}}(n h)\right] / h$, which is positive whenever more particles leave the reservoir $i$ towards the QD on average, and negative otherwise. By averaging $J_{\mathrm{i}}(n h)$ over a full cycle we have that $\bar{J}_{\mathrm{H}}=(1 / \tau) \sum_{n=0}^{\tau_{\mathrm{H}} / h-1} J_{\mathrm{H}}(n h) h$ and $\bar{J}_{\mathrm{C}}=$ $(1 / \tau) \sum_{m=\tau_{\mathrm{H}} / h}^{\tau / h-1} J_{\mathrm{C}}(m h) h$, for $i \in\{\mathrm{H}, \mathrm{C}\}$. Since no electron accumulation in the QD is possible, all particles leaving a given reservoir must go to the other one, such that $\bar{J}_{\mathrm{H}}+\bar{J}_{\mathrm{C}}=0$. By considering the master equation regime, $h \rightarrow 0$ and $n \rightarrow \infty$ with $n h=t$ held fixed, the above probabilities and currents in the NESS become

$$
\begin{gathered}
p_{\mathrm{H}}(t)=\frac{\omega_{\mathrm{H}}}{\omega_{\mathrm{H}}+\bar{\omega}_{\mathrm{H}}}-\frac{e^{-\left(\omega_{\mathrm{H}}+\bar{\omega}_{\mathrm{H}}\right) t}\left[1-e^{-\left(\omega_{\mathrm{C}}+\bar{\omega}_{\mathrm{C}}\right)\left(\tau-\tau_{\mathrm{H}}\right)}\right]}{1-e^{-\left(\omega_{\mathrm{H}}+\bar{\omega}_{\mathrm{H}}\right) \tau_{\mathrm{H}}-\left(\omega_{\mathrm{C}}+\bar{\omega}_{\mathrm{C}}\right)\left(\tau-\tau_{\mathrm{H}}\right)}} \frac{\omega_{\mathrm{H}} \bar{\omega}_{\mathrm{C}}-\bar{\omega}_{\mathrm{H}} \omega_{\mathrm{C}}}{\left(\omega_{\mathrm{H}}+\bar{\omega}_{\mathrm{H}}\right)\left(\omega_{\mathrm{C}}+\bar{\omega}_{\mathrm{C}}\right)}, \quad t=\left[0, \tau_{\mathrm{H}}\right](\bmod \tau), \\
p_{\mathrm{C}}(t)=\frac{\omega_{\mathrm{C}}}{\omega_{\mathrm{C}}+\bar{\omega}_{\mathrm{C}}}-\frac{e^{-\left(\omega_{\mathrm{C}}+\bar{\omega}_{\mathrm{C}}\right)\left(t-\tau_{\mathrm{H}}\right)}\left[1-e^{-\left(\omega_{\mathrm{H}}+\bar{\omega}_{\mathrm{H}}\right) \tau_{\mathrm{H}}}\right]}{1-e^{-\left(\omega_{\mathrm{H}}+\bar{\omega}_{\mathrm{H}}\right) \tau_{\mathrm{H}}-\left(\omega_{\mathrm{C}}+\bar{\omega}_{\mathrm{C}}\right)\left(\tau-\tau_{\mathrm{H}}\right)}} \frac{\bar{\omega}_{\mathrm{H}} \omega_{\mathrm{C}}-\omega_{\mathrm{H}} \bar{\omega}_{\mathrm{C}}}{\left(\omega_{\mathrm{H}}+\bar{\omega}_{\mathrm{H}}\right)\left(\omega_{\mathrm{C}}+\bar{\omega}_{\mathrm{C}}\right)}, \quad t=\left[\tau_{\mathrm{H}}, \tau\right](\bmod \tau), \\
\bar{J}_{\mathrm{H}}=-\bar{J}_{\mathrm{C}}=\frac{1}{\tau} \frac{\left(1-e^{\left.-\left(\omega_{\mathrm{H}}+\bar{\omega}_{\mathrm{H}}\right) \tau_{\mathrm{H}}\right)\left(1-e^{-\left(\omega_{\mathrm{C}}+\bar{\omega}_{\mathrm{C}}\right)\left(\tau-\tau_{\mathrm{H}}\right)}\right)} \frac{\omega_{\mathrm{H}} \bar{\omega}_{\mathrm{C}}-\bar{\omega}_{\mathrm{H}} \omega_{\mathrm{C}}}{1-e^{-\left(\omega_{\mathrm{H}}+\bar{\omega}_{\mathrm{H}}\right) \tau_{\mathrm{H}}-\left(\omega_{\mathrm{C}}+\bar{\omega}_{\mathrm{C}}\right)\left(\tau-\tau_{\mathrm{H}}\right)}} \cdot\right.}{\left(\omega_{\mathrm{H}}+\bar{\omega}_{\mathrm{H}}\right)\left(\omega_{\mathrm{C}}+\bar{\omega}_{\mathrm{C}}\right)} .
\end{gathered}
$$


We pause to make a few comments: First, such results recover the findings from Refs. [33,38] for symmetric interaction times $\left(\tau_{H}=\tau / 2\right)$. Second, for both discrete and continuous cases, the hot reservoir is "more willing" to concede particles than the cold reservoir when $\omega_{\mathrm{H}} / \bar{\omega}_{\mathrm{H}}>\omega_{\mathrm{C}} / \bar{\omega}_{\mathrm{C}}$, implying that $\bar{J}_{\mathrm{H}}>0$ and $\bar{J}_{\mathrm{C}}<0$, which is consistent with the fact that $\bar{J}_{\mathrm{i}}$ points from reservoir $i$ to the QD. Third, the period $\tau$ only gives the time scale of the model in the sense that rescaling the fluxes, transition rates, and the time the system stays in contact with each reservoir by $\tau$ keeps Eq. (6) unchanged; hence, from now on, $\tau$ is kept fixed and reads $\tau=1$. Fourth and last, the present system can operate as a heat engine, refrigerator, heater, or accelerator, provided that the parameters $\omega_{i}$ and $\bar{\omega}_{i}$ (or equivalently, $\epsilon, \mu_{i}$, and $T_{i}$ ) are conveniently adjusted. In this paper we shall address the heat engine regime, which is set by the conditions $\left\{\omega_{\mathrm{C}} / \bar{\omega}_{\mathrm{C}}<\omega_{\mathrm{H}} / \bar{\omega}_{\mathrm{H}}<1, \mu_{\mathrm{H}}<\mu_{\mathrm{C}}<\right.$ $\epsilon$, ensuring the positiveness of temperatures (with $T_{\mathrm{H}}>T_{\mathrm{C}}$ ), power output, and the heat extracted from the hot reservoir.

\section{THERMODYNAMICS}

Once the probability distribution and the suited heat engine regime in terms of the model parameters have been obtained, we are in a position to obtain the thermodynamic properties (exchanged heat and work) and efficiency through the framework of stochastic thermodynamics [11,39]. Remarkable quantities averaged over a complete cycle are the exchanged heat and the chemical work given by $\overline{\dot{Q}}_{i} \equiv(\epsilon-$ $\left.\mu_{i}\right) \bar{J}_{i}$ and $\bar{W}_{i}^{\text {chem }} \equiv \mu_{i} \bar{J}_{i}$, respectively, for $i=\{\mathrm{C}, \mathrm{H}\}$. It is worth mentioning that they obey the first law of thermodynamics, in such a way that $\overline{\dot{Q}}_{\mathrm{C}}+\overline{\dot{Q}}_{\mathrm{H}}+\overline{\dot{W}}_{\mathrm{C}}^{\text {chem }}+\overline{\dot{W}}_{\mathrm{H}}^{\text {chem }}=0$. For the engine regime, the efficiency is typically a measure of "what you get and what you give," represented here by the ratio between the power output $\bar{P} \equiv-\left(\overline{\dot{W}}_{\mathrm{H}}^{\text {chem }}+\overline{\dot{W}}_{\mathrm{C}}^{\text {chem }}\right)$ and the heat received from the hot reservoir $\bar{Q}_{\mathrm{H}}$, resulting in

$$
\eta \equiv \frac{\bar{P}}{\dot{Q}_{\mathrm{H}}}=1-\frac{T_{\mathrm{C}} \ln \omega_{\mathrm{C}} / \bar{\omega}_{\mathrm{C}}}{T_{\mathrm{H}} \ln \omega_{\mathrm{H}} / \bar{\omega}_{\mathrm{H}}}
$$

The relation above can also be expressed in terms of the macroscopic properties of the reservoirs and the QD

$$
\eta=1-\frac{\mu_{\mathrm{C}}-\epsilon}{\mu_{\mathrm{H}}-\epsilon}=\frac{\mu_{\mathrm{C}}-\mu_{\mathrm{H}}}{\epsilon-\mu_{\mathrm{H}}},
$$

where we used Eq. (1). We pause again to make some comments: First, the system will reach an equilibrium state when $\omega_{\mathrm{H}} / \bar{\omega}_{\mathrm{H}}=\omega_{\mathrm{C}} / \bar{\omega}_{\mathrm{C}}$, consistent with the (maximum) Carnot efficiency. Second, the engine regime stated above, $\left(\omega_{\mathrm{C}} / \bar{\omega}_{\mathrm{C}}<\omega_{\mathrm{H}} / \bar{\omega}_{\mathrm{H}}<1\right),\left(\mu_{\mathrm{H}}<\mu_{\mathrm{C}}<\epsilon\right)$, ensures the positiveness of the power output and the heat delivered from the hot reservoir. Additionally, it is also consistent with heat flowing from the QD to the cold reservoir: $\bar{Q}_{\mathrm{C}}<0$. Third and last, since the right-hand side of Eq. (8) does not depend on $\tau_{\mathrm{H}}$ and $\tau, \eta$ is independent of the protocol. Conversely, the power output $\bar{P}$ depends on $\tau_{\mathrm{H}}$ and $\tau$, in such a way that it can be conveniently adjusted, together with the other parameters $\left\{\omega_{\mathrm{H}}, \bar{\omega}_{\mathrm{H}}, \omega_{\mathrm{C}}, \bar{\omega}_{\mathrm{C}}, T_{\mathrm{H}}, T_{\mathrm{C}}\right\}$, in order to optimize the extracted power. Here, we are concerned with the maximization of power under different physical setups.

In order to exploit distinct possibilities of optimizing the extracted power, the next sections will be devoted to its maximization with respect to the protocol asymmetry and its complete maximization (also taking into account the transition rates). Since the extracted power increases monotonically with the ratio between temperatures, the analysis will be carried out for finite fixed ratios $T_{\mathrm{C}} / T_{\mathrm{H}}$.

\section{A. Best protocol}

An interesting way of obtaining some insight into the system is to look at the power output as a function of the fraction of time spent in contact with the hot reservoir $\tau_{\mathrm{H}}$ and the ratio between the duration of the cycle and the characteristic time $t_{\text {char }}$ [defined as the largest of characteristic times $t_{\text {char }}^{\mathrm{H}} \equiv$ $1 /\left(\omega_{\mathrm{H}}+\bar{\omega}_{\mathrm{H}}\right)$ and $t_{\text {char }}^{\mathrm{C}} \equiv 1 /\left(\omega_{\mathrm{C}}+\bar{\omega}_{\mathrm{C}}\right)$, which represent the typical relaxation time to the thermal state that each reservoir imposes] as exemplified in Fig. 2, for some representative parameter values. In all cases, the chemical potentials and the energy are held fixed providing $\eta=1 / 3$ [see Eq. (8) and the caption of Fig. 2 for the values used]. A common trait of all panels is that the power output (and also the extracted heat) vanishes for $\tau_{\mathrm{H}} / \tau$ near 1 or 0 . This is expected since in such a situation the QD is mostly in contact with a single reservoir and almost no work can be extracted. It is also noteworthy that the value of $\tau_{\mathrm{H}}$ for which the power is maximum barely changes with the ratio $\tau / t_{\text {char }}$ but its value is extremely dependent on the other parameters of the model. Furthermore, even for a constant value of $\tau / t_{\text {char }}$, the power output can be noticeably different.

In order for the reservoir to effectively act upon the QD state it needs to interact during a period comparable with its characteristic time. Therefore characteristic times provide a notion of how long a good engine will interact with each reservoir; for example, if $t_{\text {char }}^{\mathrm{H}} \ll t_{\text {char }}^{\mathrm{C}}$, the hot reservoir needs to interact very briefly compared with the cold one, so we can expect that a good protocol will present a small $\tau_{\mathrm{H}} / \tau$. Hence it is reasonable that good performance protocols will present interaction times around the characteristic times of each reservoir. This is exemplified in Fig. 2, where $t_{\text {char }}^{\mathrm{H}} /\left(t_{\text {char }}^{\mathrm{H}}+t_{\text {char }}^{\mathrm{C}}\right)$ gives approximately $0.7,0.1,0.9$, and 0.4 for Figs. 2(a), 2(b), 2(c), and 2(d), respectively, in fair agreement with the best value of $\tau_{\mathrm{H}} / \tau$ observed in the plots. As a rule of thumb we should choose $\tau$ as small as possible, in agreement with Refs. [40,41], and maximize the power output with respect to $\tau_{\mathrm{H}}$. Although small, it is worth mentioning that the period $\tau$ should be sufficiently larger than the time necessary for switching the interaction of the QD from one reservoir to the other one, in similarity with the symmetric case $[33,35,35]$.

The unique value of $\tau_{\mathrm{H}}$ that maximizes $\bar{P}$ for a given value of $\tau$ and the other parameters can be easily found by numerically solving the transcendental equation

$$
\frac{\left(\omega_{\mathrm{H}}+\bar{\omega}_{\mathrm{H}}\right)}{\left(\omega_{\mathrm{C}}+\bar{\omega}_{\mathrm{C}}\right)} \frac{e^{-\left(\omega_{\mathrm{H}}+\bar{\omega}_{\mathrm{H}}\right) \tau_{\mathrm{H}}}\left[1-e^{-\left(\omega_{\mathrm{C}}+\bar{\omega}_{\mathrm{C}}\right)\left(\tau-\tau_{\mathrm{H}}\right)}\right]^{2}}{e^{-\left(\omega_{\mathrm{C}}+\bar{\omega}_{\mathrm{C}}\right)\left(\tau-\tau_{\mathrm{H}}\right)}\left[1-e^{-\left(\omega_{\mathrm{H}}+\bar{\omega}_{\mathrm{H}}\right) \tau_{\mathrm{H}}}\right]^{2}}=1,
$$

whose values are shown in Fig. 2, represented by the white dashed curves. 


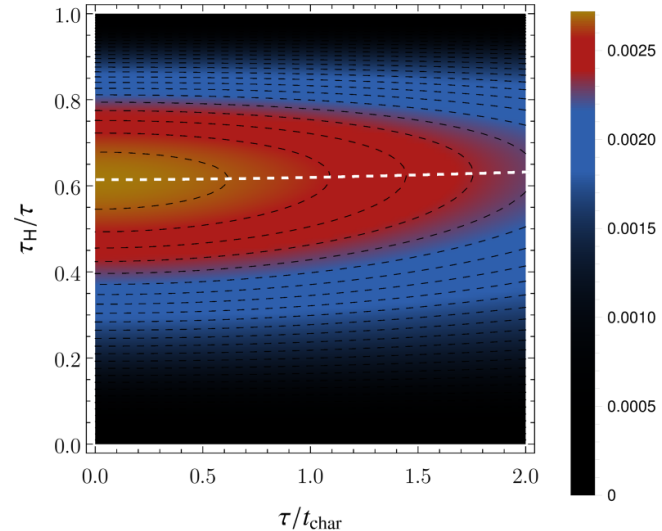

(a) $T_{\mathrm{H}}=0.4, T_{\mathrm{C}}=0.1, \omega_{\mathrm{H}}=0.04, \bar{\omega}_{\mathrm{H}}=0.18, \omega_{\mathrm{C}}=0.01$ and $\bar{\omega}_{\mathrm{C}}=0.55$.

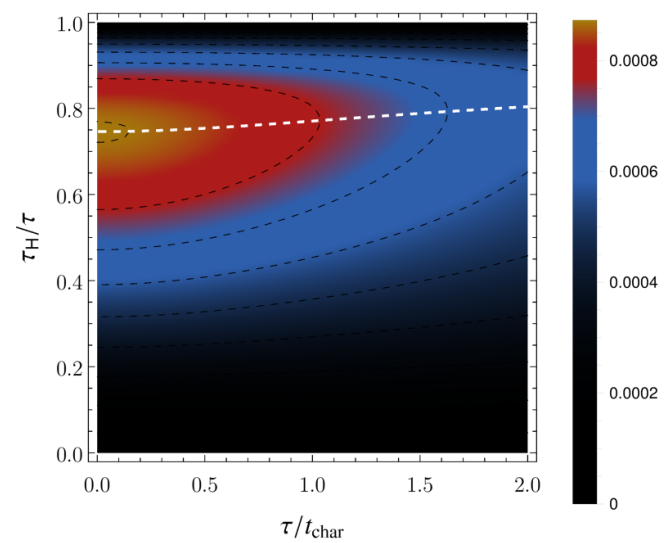

(c) $T_{\mathrm{H}}=3.5, T_{\mathrm{C}}=1.2, \omega_{\mathrm{H}}=0.09, \bar{\omega}_{\mathrm{H}}=0.11, \omega_{\mathrm{C}}=0.71$ and $\bar{\omega}_{\mathrm{C}}=0.99$.

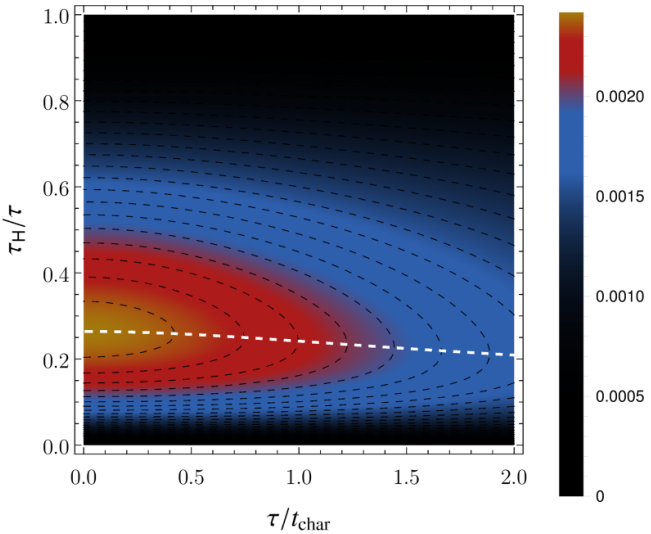

(b) $T_{\mathrm{H}}=2.0, T_{\mathrm{C}}=0.5, \omega_{\mathrm{H}}=0.64, \bar{\omega}_{\mathrm{H}}=0.86, \omega_{\mathrm{C}}=0.06$ and $\bar{\omega}_{\mathrm{C}}=0.13$.

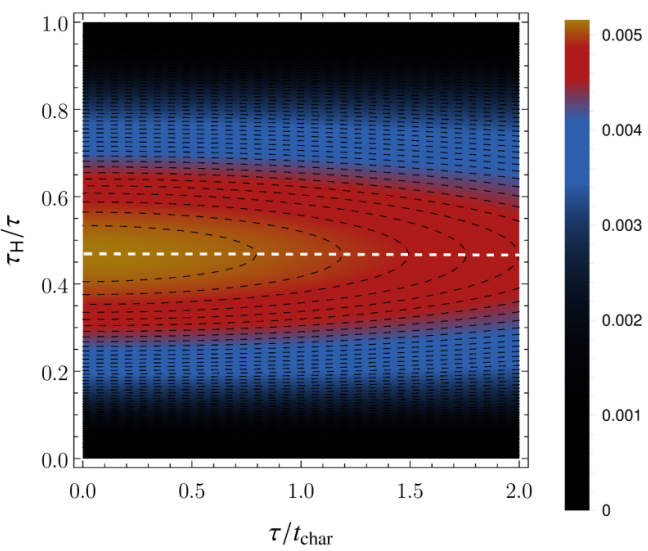

(d) $T_{\mathrm{H}}=0.4, T_{\mathrm{C}}=0.1, \omega_{\mathrm{H}}=0.13, \bar{\omega}_{\mathrm{H}}=0.58, \omega_{\mathrm{C}}=0.01$ and $\bar{\omega}_{\mathrm{C}}=0.55$.

FIG. 2. (a)-(d) Power outputs vs $\tau / t_{\text {char }}$ and $\tau_{\mathrm{H}} / \tau$ for distinct control parameters. Each dashed black curve represents an increment of $10^{-4}$ in the value of the power, and the white dashed curves represent the value of $\tau_{\mathrm{H}} / \tau$ that maximizes the power for each value of $\tau / t_{\mathrm{char}}$. For all panels, we set $\mu_{\mathrm{H}}=0.4, \mu_{\mathrm{C}}=0.6$, and energy $\epsilon=1$.

An interesting question that may be raised about this best protocol procedure is, How much do we gain by tuning $\tau_{\mathrm{H}}$ instead of just letting the QD be half of the time in contact with each reservoir (symmetric case)? In Fig. 3, we show the density plots of the ratio of the power output for $\tau_{\mathrm{H}}$ to the power output for the symmetric case $\bar{P}\left(\tau_{\mathrm{H}}\right) / \bar{P}(\tau / 2)$ as a function of both $\tau_{\mathrm{H}} / \tau$ and $T_{\mathrm{C}} / T_{\mathrm{H}}$. As the temperature is changed (with $\bar{\omega}_{\mathrm{i}}$ 's, $\epsilon$, and the chemical potentials held fixed), $t_{\text {char }}$ changes according to Eq. (1). Hence comparisons between Figs. 2 and 3 should take this change into account.

Here, we used the same values of chemical potentials and energy as in Fig. 2. As can be seen, by properly tuning $\tau_{\mathrm{H}}$ one can increase the power output more than $25 \%$ [Figs. 3(a) and 3(e)]. However, in order for the tuning be more effective, the transition rates $\bar{\omega}_{\mathrm{H}}$ and $\bar{\omega}_{\mathrm{C}}$ must be distinct, adding an asymmetry to the system: Figs. 3(c) and 3(d) reveal that the ratio $\bar{P}\left(\tau_{\mathrm{H}}\right) / \bar{P}(\tau / 2)$ is less than or equal to 1 for $\bar{\omega}_{\mathrm{H}}=\bar{\omega}_{\mathrm{C}}$. Finally, by comparing Fig. 3(a) with Fig. 3(b) and Fig. 3(e) with Fig. 3(f) we notice that the tuning of $\tau_{\mathrm{H}}$ can increase the power output for $\bar{\omega}_{\mathrm{H}}$ either greater or smaller than $\bar{\omega}_{\mathrm{C}}$, but it is for $\bar{\omega}_{\mathrm{H}}>\bar{\omega}_{\mathrm{C}}$ that the region of larger gain corresponds to the larger efficiencies and power outputs (small $T_{\mathrm{C}} / T_{\mathrm{H}}$ ).

\section{B. Complete maximization of power}

In this section, the complete maximization of the power, not only with respect to $\tau_{\mathrm{H}}$ (as in the previous section) but also taking into account the transition rates, is undertaken. Physically, this means that we are optimizing the physical properties (transition rates) as well as the machine protocol (time spent in contact with each reservoir). Some remarkable features are depicted in Fig. 4, in which the parameters $\tau_{\mathrm{H}} / \tau$, $\omega_{\mathrm{H}}$, and $\omega_{\mathrm{C}}$ that maximize the power output are considered as a function of the ratio $T_{\mathrm{C}} / T_{\mathrm{H}}$. That is, the optimization of the power output (with respect to $\tau_{\mathrm{H}}, \omega_{\mathrm{H}}$, and $\omega_{\mathrm{C}}$ ) is undertaken for all values of $\bar{\omega}_{H}$ and $\bar{\omega}_{\mathrm{C}}$ in a square grid (from 0.1 to 2 in steps of 0.05). The maximum, mean, and minimum values of these quantities are then shown in Fig. 4. Although the ratios $\omega_{\mathrm{H}} / \bar{\omega}_{\mathrm{H}}$ and $\omega_{\mathrm{C}} / \bar{\omega}_{\mathrm{C}}$ vary slightly with the choice of the rates $\bar{\omega}_{\mathrm{H}}$ and $\bar{\omega}_{\mathrm{C}}$, an opposite trend is verified for $\tau_{\mathrm{H}}$, which is very sensitive to the choice of transition rates or grids. This suggests that the choice of the time fraction which the QD spends in contact with each reservoir is the most important parameter for the power maximization. Such a finding is reinforced by examining the behavior of output powers in Fig. 5 in which, in addition to the optimal choices of $\omega_{\mathrm{H}}$ and $\omega_{\mathrm{C}}$, one also takes "reliable" estimates for them and only the optimization 


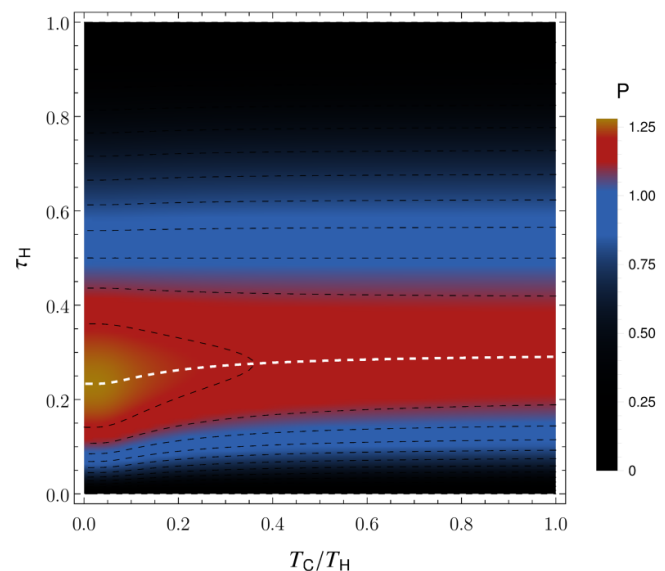

(a) $T_{\mathrm{H}}=2.0, \bar{\omega}_{\mathrm{H}}=0.86$ and $\bar{\omega}_{\mathrm{C}}=0.14$.

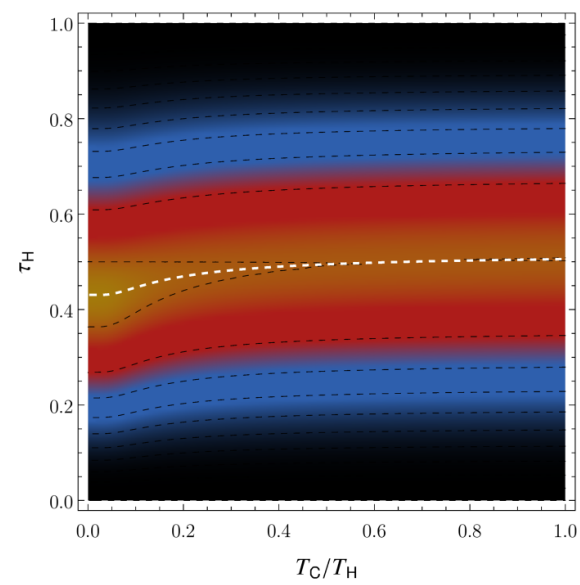

(c) $T_{\mathrm{H}}=2.0, \bar{\omega}_{\mathrm{H}}=0.5$ and $\bar{\omega}_{\mathrm{C}}=0.5$.

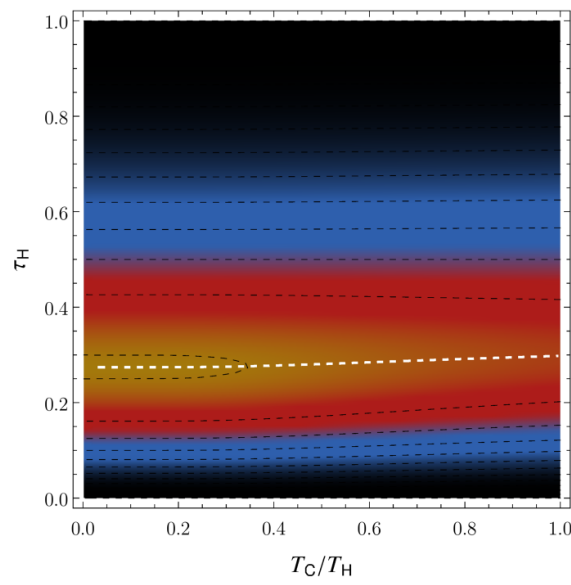

$(\mathrm{e}) T_{\mathrm{H}}=0.3, \bar{\omega}_{\mathrm{H}}=0.86$ and $\bar{\omega}_{\mathrm{C}}=0.14$.

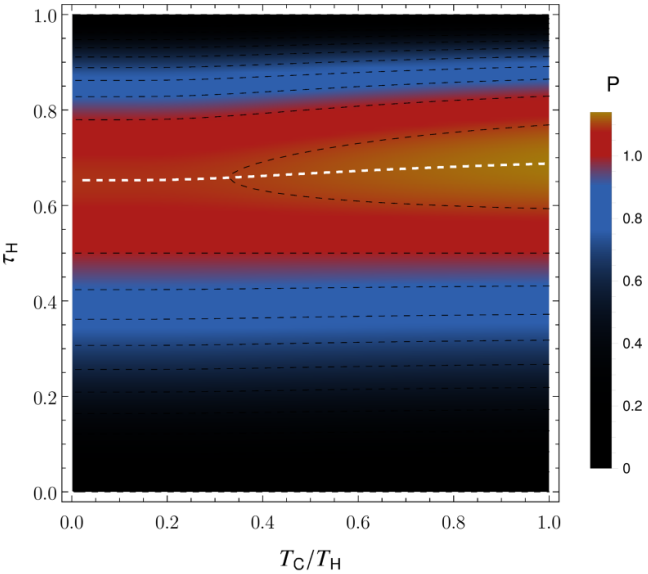

(b) $T_{\mathrm{H}}=0.4, \bar{\omega}_{\mathrm{H}}=0.16$ and $\bar{\omega}_{\mathrm{C}}=0.69$.

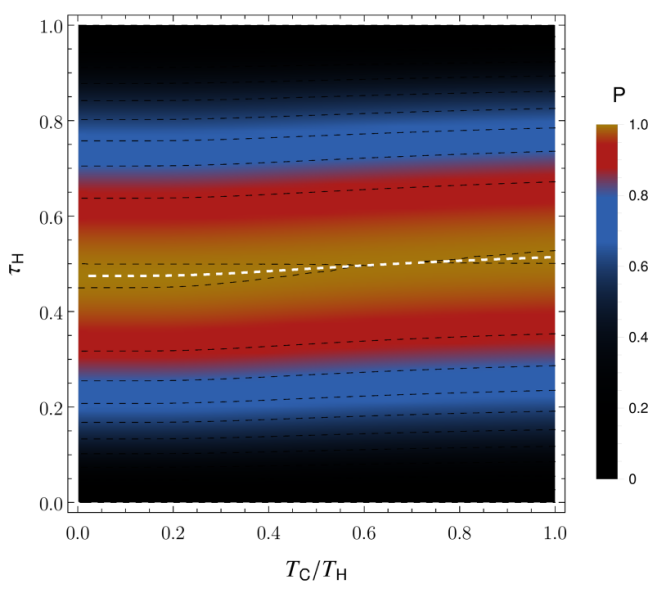

(d) $T_{\mathrm{H}}=0.4, \bar{\omega}_{\mathrm{H}}=0.5$ and $\bar{\omega}_{\mathrm{C}}=0.5$.

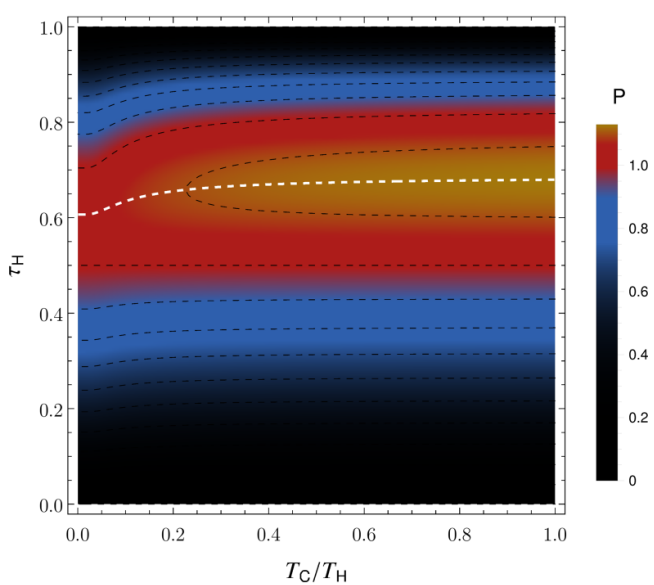

(f) $T_{\mathrm{H}}=3.0, \bar{\omega}_{\mathrm{H}}=0.16$ and $\bar{\omega}_{\mathrm{C}}=0.69$.

FIG. 3. (a)-(f) Density plots of the power as a function of $\tau_{\mathrm{H}}$ and $T_{\mathrm{C}} / T_{\mathrm{H}}$ normalized by the power for $\tau_{\mathrm{H}}=\tau / 2$. Each dashed black curve represents an increment of 0.1 in the value of the power, and the white dashed curves represent the value of $\tau_{\mathrm{H}}$ that maximizes the power for each value of $T_{\mathrm{C}} / T_{\mathrm{H}}$. For all panels, we set $\tau=1, \mu_{\mathrm{H}}=0.4, \mu_{\mathrm{C}}=0.6$, and $\epsilon=1$.

with respect to $\tau_{\mathrm{H}}$ is carried out. Although the power output strongly depends on the choices of rates or grids $\bar{\omega}_{H}$ and $\bar{\omega}_{\mathrm{C}}$ (see, e.g., the lowest and largest $\bar{P}_{\text {max }}$ curves), the difference between power outputs coming from the optimization of $\omega_{\mathrm{i}}$ and their mean values is very small (not visible in the scale of Fig. 5). Finally, the difference between the lowest and largest $\bar{P}_{\max }$ is due to its monotonic increasing by raising the transition rates.

Lastly, the efficiency at maximal power $\eta_{\mathrm{MP}}$ is compared with the well-established Curzon-Ahlborn and Carnot ones in Figs. 6 and 7, respectively, for different temperatures. Just as in Figs. 4 and 5, the optimization is carried out for all values 


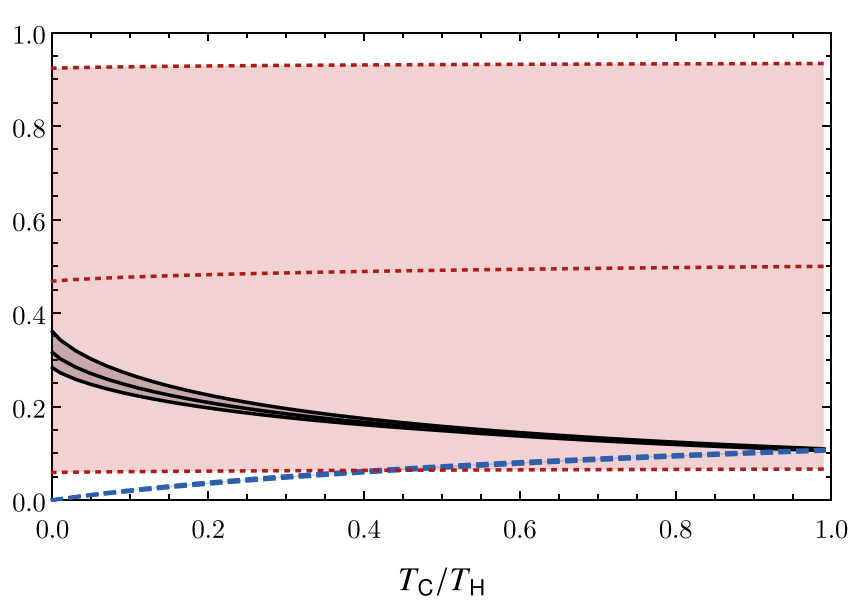

FIG. 4. Values of $\tau_{\mathrm{H}} / \tau$ (red dashed curves), $\omega_{\mathrm{H}} / \bar{\omega}_{\mathrm{H}}$ (black solid curves), and $\omega_{\mathrm{C}} / \bar{\omega}_{\mathrm{C}}$ (blue dashed curve) after the maximization of power for fixed temperatures and $\tau=1$. The maximum, medium, and minimum values for each quantity are presented, and the highlighted areas between the three curves represent the possible values of each parameter after the optimization of the power for a grid of values of $\bar{\omega}_{\mathrm{H}}$ and $\bar{\omega}_{\mathrm{C}}$ ranging from 0.1 to 2 in steps of 0.05 .

of $\bar{\omega}_{\mathrm{H}}$ and $\bar{\omega}_{\mathrm{C}}$ over a grid. Except for some specific choices of $\bar{\omega}_{\mathrm{H}}$ and $\bar{\omega}_{\mathrm{C}}$ in a small range of $T_{\mathrm{C}} / T_{\mathrm{H}}$ between 0.4 and 1 , the maximization provides an efficiency slightly larger than the Curzon-Ahlborn one. As for Carnot and Curzon-Ahlborn efficiencies, Eqs. (1) and (7) show that $\eta_{\text {MP }}$ solely depends on the ratio of the temperatures and not on the specific value of $T_{\mathrm{H}}$.

Finally, $\eta_{\mathrm{MP}}$ is always (as it must be) lower than $\eta_{\mathrm{C}}$ (see, e.g., Fig. 7) for all of the set of optimized parameters. Furthermore, when $T_{\mathrm{C}} / T_{\mathrm{H}} \rightarrow 0$ and 1 , all efficiencies collapse at the asymptotic values 1 and 0 , respectively.

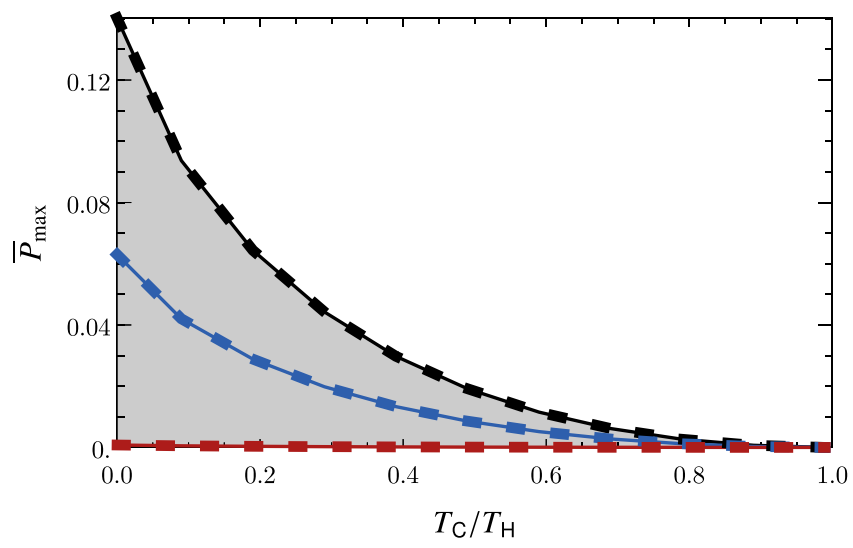

FIG. 5. Maximum power output $\bar{P}_{\max }$ vs $T_{\mathrm{C}} / T_{\mathrm{H}}$ for $\tau=1$ and the same grid used in Fig. 4, where maximum (black), mean (blue), and minimum (red) values of maximum power are displayed. Solid curves account for the maximization in terms of $\tau_{\mathrm{H}}$ only, while dashed curves represent the maximization in terms of $\tau_{\mathrm{H}}, \omega_{\mathrm{H}} / \bar{\omega}_{\mathrm{H}}$, and $\omega_{\mathrm{C}} / \bar{\omega}_{\mathrm{C}}$.

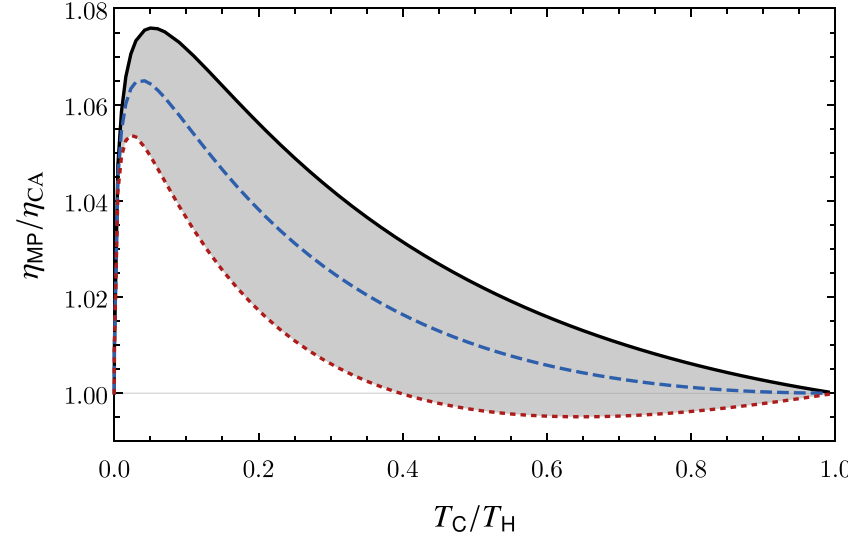

FIG. 6. Ratio between the efficiency at maximum power $\eta_{\mathrm{MP}}$ and the Curzon-Ahlborn efficiency $\eta_{\mathrm{CA}}$ vs $T_{\mathrm{C}} / T_{\mathrm{H}}$ for $\epsilon=1$ and $\tau=1$. The curves represent the loci of maximum (black solid curve), mean (blue dashed curve), and minimum (red dashed curve) values obtained for the same grid used in Fig. 4.

\section{DISCUSSION}

In this paper, we analyzed the role of asymmetric interaction times for optimizing the power output in thermal machines composed of a quantum dot stochastic pump. Our findings showed that a suited power output optimization can lead to a gain in the power larger than $25 \%$ when compared with the symmetrical case, in which the system is equally placed in contact with each reservoir.

A very remarkable point regarding the importance of the present analysis is that the fine-tuning of the interaction time is expected to be easier to implement than an improved design of the machine itself. Therefore the present (exact) results may shed some light on the role of the time protocol in the effectiveness of obtaining optimized power outputs. In fact, the choice of a "good" (instead of optimal) quantum dot

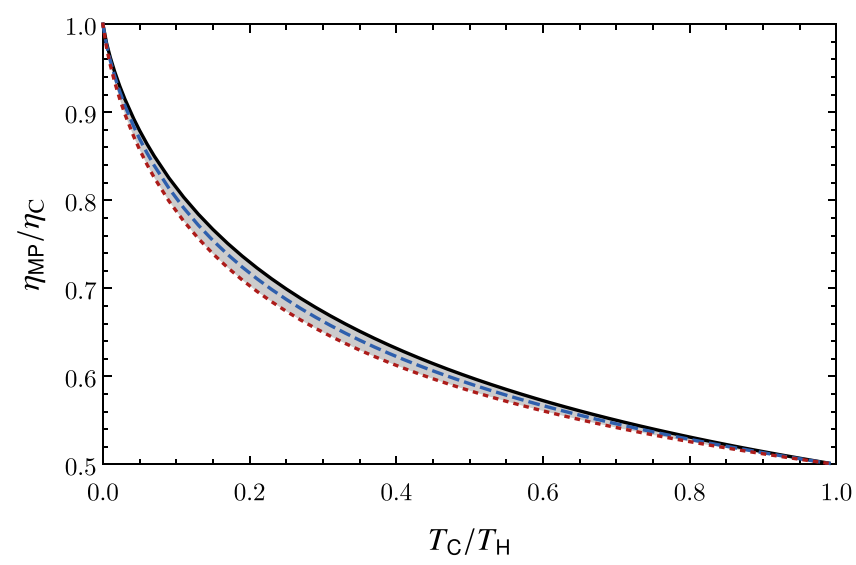

FIG. 7. Ratio between efficiency at maximum power $\eta_{\mathrm{MP}}$ and Carnot $\eta_{\mathrm{C}}$ vs $T_{\mathrm{C}} / T_{\mathrm{H}}$ for $\epsilon=1$ and $\tau=1$. The curves represent the loci of maximum (black solid curve), mean (blue dashed curve), and minimum (red dashed curve) values obtained for the same grid used in Fig. 4. 
together with optimized time provides a reliable recipe for obtaining an almost optimal (power output) machine, whose associate efficiencies are usually somewhat larger than the Curzon-Ahlborn one, but much larger than the symmetric case.

Further investigations of this model could explore other regimes (e.g., as a refrigerator or a heater engine) and/or the modulation of the QD's energy to obtain mechanical work on top of the pumping of electrons.

\section{ACKNOWLEDGMENTS}

P.E.H. acknowledges Grants No. 2017/24567-0 and No. 2020/03708-8, São Paulo Research Foundation (FAPESP). C.E.F. acknowledges Grant No. 2018/02405-1 (FAPESP). A.R. is thankful for Pronex/Fapesq-PB/CNPq Grant No. $151 / 2018$ and CNPq Grant No. 308344/2018-9. This study was financed in part by the Coordenação de Aperfeiçoamento de Pessoal de Nível Superior, Brasil (CAPES), Finance Code 001 .
[1] S. Carnot, in Annales Scientifiques de l'École Normale Supérieure (École Normale Supérieure, Paris, 1872), Vol. 1, pp. 393-457.

[2] M. J. De Oliveira, Equilibrium Thermodynamics (Springer, Heidelberg, 2013).

[3] G. Verley, M. Esposito, T. Willaert, and C. Van den Broeck, Nat. Commun. 5, 4721 (2014).

[4] I. A. Martínez, É. Roldán, L. Dinis, D. Petrov, J. M. Parrondo, and R. A. Rica, Nat. Phys. 12, 67 (2016).

[5] S. Vinjanampathy and J. Anders, Contemp. Phys. 57, 545 (2016).

[6] R. Kosloff, Entropy 15, 2100 (2013).

[7] F. Curzon and B. Ahlborn, Am. J. Phys. 43, 22 (1975).

[8] I. Novikov, J. Nucl. Energy 7, 125 (1958).

[9] C. Van den Broeck, Phys. Rev. Lett. 95, 190602 (2005).

[10] T. Schmiedl and U. Seifert, EPL 81, 20003 (2007).

[11] U. Seifert, Rep. Prog. Phys. 75, 126001 (2012).

[12] M. Esposito, K. Lindenberg, and C. Van den Broeck, Phys. Rev. Lett. 102, 130602 (2009).

[13] B. Cleuren, B. Rutten, and C. Van den Broeck, Eur. Phys. J.: Spec. Top. 224, 879 (2015).

[14] M. Esposito, R. Kawai, K. Lindenberg, and C. Van den Broeck, Phys. Rev. E 81, 041106 (2010).

[15] U. Seifert, Phys. Rev. Lett. 106, 020601 (2011).

[16] Y. Izumida and K. Okuda, EPL 97, 10004 (2012).

[17] N. Golubeva and A. Imparato, Phys. Rev. Lett. 109, 190602 (2012).

[18] V. Holubec, J. Stat. Mech. (2014) P05022.

[19] M. Bauer, K. Brandner, and U. Seifert, Phys. Rev. E 93, 042112 (2016).

[20] K. Proesmans, B. Cleuren, and C. Van den Broeck, Phys. Rev. Lett. 116, 220601 (2016).

[21] Z. Tu, J. Phys. A: Math. Theor. 41, 312003 (2008).

[22] S. Ciliberto, Phys. Rev. X 7, 021051 (2017).
[23] B. A. N. Akasaki, M. J. de Oliveira, and C. E. Fiore, Phys. Rev. E 101, 012132 (2020).

[24] T. Tomé and M. J. de Oliveira, Phys. Rev. E 91, 042140 (2015).

[25] C. Van den Broeck, Nat. Nanotechnol. 13, 876 (2018).

[26] H. Scovil and E. Schulz-DuBois, Phys. Rev. Lett. 2, 262 (1959).

[27] M. Josefsson, A. Svilans, A. M. Burke, E. A. Hoffmann, S. Fahlvik, C. Thelander, M. Leijnse, and H. Linke, Nat. Nanotechnol. 13, 920 (2018).

[28] A. L. L. Stable, C. E. Fernandez Noa, W. G. C. Oropesa, and C. E. Fiore, Phys. Rev. Research 2, 043016 (2020).

[29] F. L. S. Rodrigues, G. De Chiara, M. Paternostro, and G. T. Landi, Phys. Rev. Lett. 123, 140601 (2019).

[30] O. A. D. Molitor and G. T. Landi, Phys. Rev. A 102, 042217 (2020).

[31] J. M. Parrondo, J. M. Horowitz, and T. Sagawa, Nat. Phys. 11, 131 (2015).

[32] P. Strasberg, G. Schaller, T. Brandes, and M. Esposito, Phys. Rev. X 7, 021003 (2017).

[33] A. Rosas, C. Van den Broeck, and K. Lindenberg, Phys. Rev. E 96, 052135 (2017).

[34] A. Rosas, C. Van den Broeck, and K. Lindenberg, Phys. Rev. E 97, 062103 (2018).

[35] S. H. Lee, J. Um, and H. Park, Phys. Rev. E 98, 052137 (2018).

[36] S. Mondal, S. Bhattacharjee, and A. Dutta, Phys. Rev. E 102, 022140 (2020).

[37] V. Cavina, P. A. Erdman, P. Abiuso, L. Tolomeo, and V. Giovannetti, arXiv:2010.00586.

[38] P. E. Harunari, C. E. Fiore, and K. Proesmans, J. Phys. A: Math. Theor. 53, 374001 (2020).

[39] C. Van den Broeck and M. Esposito, Phys. A (Amsterdam) 418, 6 (2015)

[40] P. A. Erdman, V. Cavina, R. Fazio, F. Taddei, and V. Giovannetti, New J. Phys. 21, 103049 (2019).

[41] V. Mukherjee, A. G. Kofman, and G. Kurizki, Commun. Phys. 3, 8 (2020). 


\section{Bibliografia}

[1] L. Onsager, Reciprocal Relations In Irreversibles Processes. I., Physical Review, vol. 37,1931

[2] I. Prigogine, Introduction to Thermodynamics of Irreversible Processes, 2nd ed. (Wiley, New York, 1961).

[3] F. L. S. Rodrigues, G. De Chiara, M. Paternostro and G. T. Landi, Thermodynamics of Weakly Coherent Collisional Models, Phys. Rev. L. 123, 140601, 2019.

[4] J. M. Parrondo, J. M. Horowitz, T. Sagawa Thermodynamics of information, Nat. Phys. 11, 131-139, 2015

[5] F.L. Curzon, B. Ahlborn, Efficiency of a Carnot engine at maximum power output, American Journal of Physics. 43: 22-24, 1975

[6] Callen, Herbert B., Thermodynamics and an Introduction to Thermostatistics, American Association of Physics Teachers, (1998).

[7] M. J. de Oliveira, Termodinâmica Livraria da Física, São Paulo, 2005.

[8] S. R. A. Salinas, Introdução à Física Estatística, EDUSP, São Paulo,2013

[9] T. Tomé e M. J. de Oliveira, Stochastic Dynamics and Irreversibility, Springer, Cham, 2015

[10] S. Katz, J. L. Lebowitz e H. Spohn, Phase Transitions in Stationary Nonequilibrium States of Model Lattice Systems, Phys. Rev. B 28, 1655(R), 1983 
[11] S. R. de Groot and P. Mazur, Non-Equilibrium Thermodynamics North-Holland, Amsterdam, 1962.

[12] S. Carnot, in Annales scientifiques de l' École Normale Supérieure, Vol. 1 (1824) Armand Colin, Paris, France, 4 1-58

[13] M. Esposito, R. Kawai, K. Lindenberg, and C. Van den Broeck, Quantum-dot Carnot engine at maximum power, Phys. Rev. E 81, 041106, 2010

[14] P. Chambadal, Les centrales nucléaires, Armand Colin, Paris, France, 4 1-58, 1957

[15] I.I. Novikov, The efficiency of atomic power stations (a review), Journal of Nuclear Energy, 1958

[16] C. Van den Broeck, Thermodynamic Efficiency at Maximum Power, Physical Review E., 95, 190602, 2005

[17] S. H. Lee, J. Um, and H. Park, Nonuniversality of heat-engine efficiency at maximum power, Phys. Rev. E., 98, 052137, 2018

[18] C. Van den Broeck, M. Esposito, Ensemble and Trajectory Thermodynamics: A Brief Introduction., Physica A, Volume 418, 15 January 2015, Pages 6-16.

[19] J. Schnakenberg, Rev. Mod. Phys. 48, 571 (1976).

[20] P. Langevin, Sur la théorie du mouvement brownien, C. R. Acad. Sci. (Paris) 146, 530-533 (1908).

[21] A. Rosas, C. Van den Broeck, and K. Lindenberg, Stochastic thermodynamics for a periodically driven single-particle pump, Phys. Rev. E 96, 052135 (2017)

[22] A. Rosas, C. Van den Broeck, and K. Lindenberg, Three-stage stochastic pump: Another type of Onsager-Casimir symmetry and results far from equilibrium, Phys. Rev. E 97, 062103 (2018).

[23] Josefsson, M., Svilans, A., Burke, A.M. et al. A quantum-dot heat engine operating close to the thermodynamic efficiency Nature Nanotech 13, 920-924 (2018). 